\title{
una solución directa para las cubiertas de hormigón en placa plegada
}

En los últimos años, debido a la comprobada economía de las construcciones en placa plegada, en la literatura americana han aparecido algunos notables artículos sobre este tema. En general, las fórmulas y ecuaciones presentadas en ellos tienen la base común de dividir el funcionamiento resistente de una placa plegada en dos acciones interdependientes, una transversal y otra longitudinal. Se establece el equilibrio de fuerzas en dirección transversal, suponiendo que unas cintas transversales genéricas están apoyadas en los puntos de unión de las placas sobre unos soportes rígidos o flexibles. La reacción que resulta de esta hipótesis se descompone en fuerzas paralelas a la placa, que, entonces, resiste estas solicitaciones como viga longitudinal. Así, pues, el problema de la placa plegada se reduce a procedimientos familiares para la mayor parte de los ingenieros, y, desde este punto de vista, el análisis es perfectamente satisfactorio.

Pero los cálculos necesarios no son tan completamente automáticos como sería de desear, especialmente cuando se realizan con largos períodos de tiempo intermedios. En bien de la facilidad de aplicación se presenta aquí un procedimiento de solución directa. El procedimiento se refiere, simplemente, al establecimiento de dos ecuaciones por cada pliegue, que expresan las relaciones entre los momentòs y las tensiones longitudinales en los puntos adyacentes y la carga actuante. La determinación de la magnitud de los momentos y de la tensión en cada punto exige la solución de un cierto número de ecuaciones simultáneas. Esta solución puede efectuarse por un procedimiento directo o por un método iterativo rápidamente convergente.

Antes de proceder al cálculo de una placa plegada, es preciso comprender el comportamiento de una estructura de este tipo. Esto puede conducir a una mejor apreciación del efecto de los distintos parámetros $\mathrm{y}$, asimismo, puede evitar cálculos innecesarios. En ciertos casos, tales como placas a una cierta distancia de los bordes libres o aquellas que incorporan miembros verticales de gran canto, no se necesita un estudio tan complejo. El cálculo de la placa plegada tratándola como viga es suficiente en estos casos.

Como cuando se trata de estructuras bidimensionales, un estudio cualitativo, y en aquel caso cuantitativo también, debe empezar con el examen de una parte de la estructura como un cuerpo aislado. En el caso de la placa plegada, el elemento aislado más apropiado es una banda transversal de anchura unidad como aparece en la figura la. Las fuerzas que actúan en este 


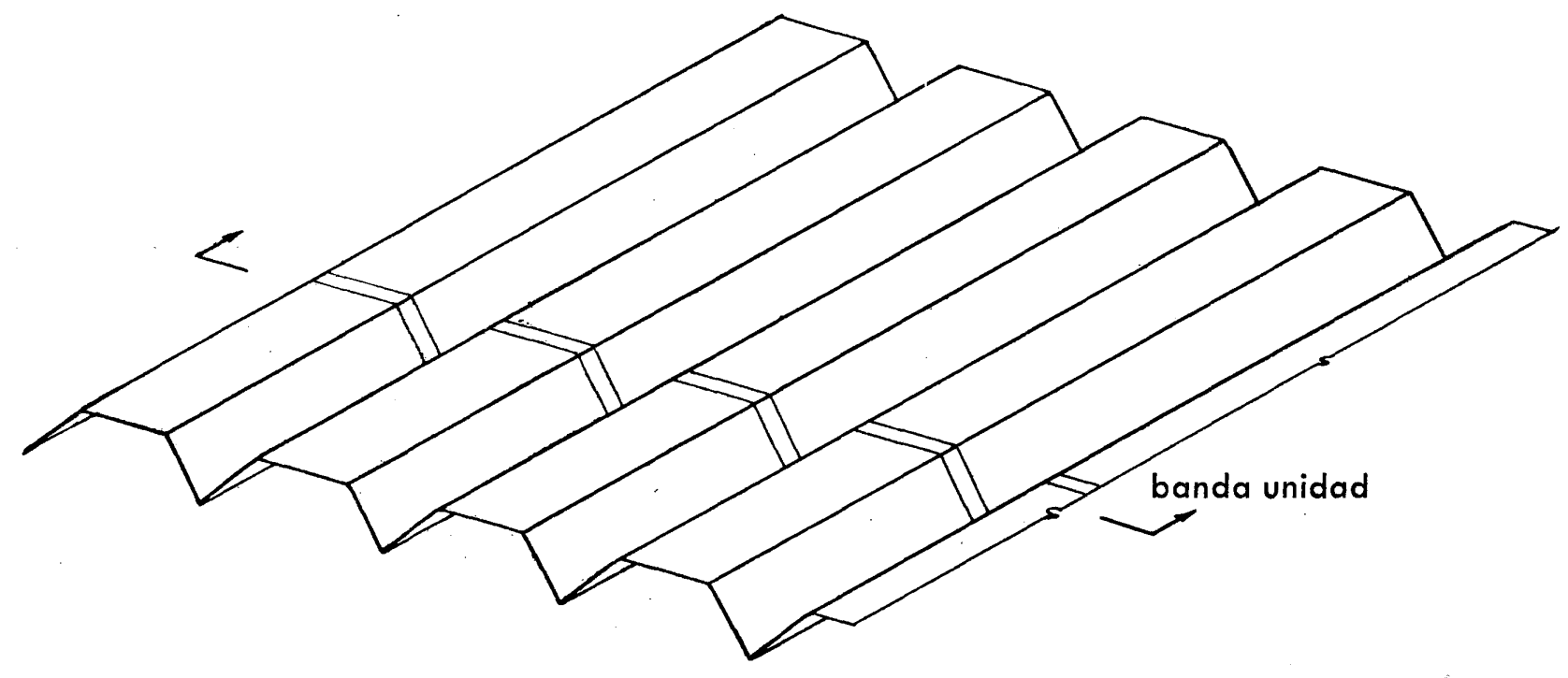

(a)
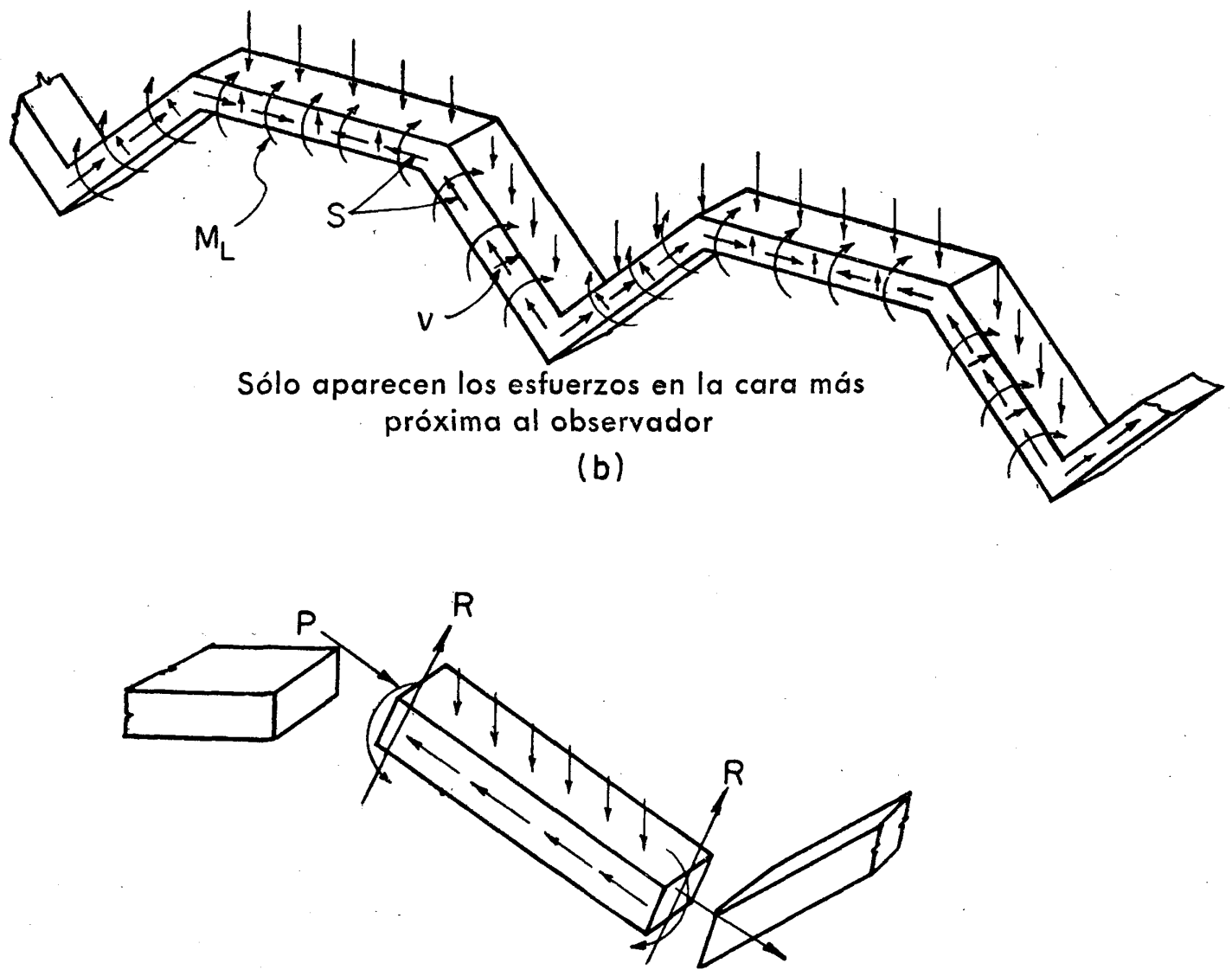

(c) 
elemento aislado, y que aparacen dibujadas a escala mayor en la figura $1 \mathrm{~b}$, están formadas por la carga exterior, los esfuerzos tangenciales variables, $s$, que actúan paralelamente a la superficie de las placas, los esfuerzos tangenciales normales, $v$, perpendiculares a las mismas y el momento $\mathrm{M}_{\mathrm{L}}$. La enorme flexibilidad de las placas en dirección perpendicular a las mismas, comparada con su rigidez en dirección paralela a las placas, hace que el valor del esfuerzo tranversal, $v$, sea insignficante. También se deduce de esto que los momentos de flexión, $\mathrm{M}$, son muy pequeños. Por esta razón, estos esfuerzos tangenciales y estos momentos pueden ser despreciados.

Al examinar más minuciosamente el elemento aislado de la figura $1 \mathrm{~b}$, aislando un solo elemento recto, como aparece en el esquema lc, se ve claramente que el momento transversal que actúa en la unión de las placas es independiente de la distribución e intensidad de los esfuerzos tangenciales que actúan sobre el elemento individual, puesto que estas fuerzas actúan paralelamente a la placa. Más aún, descomponiendo las reacciones que producen las placas adyacentes en fuerzas $\mathrm{R}$ y $\mathrm{P}$, normales y paralelas a la placa, respectivamente, las ecuaciones de equilibrio de fuerzas y momentos en un elemento individualizado son idénticas a las de un tramo de una viga continua. Por consiguiente, el comportamiento, e incluso, el estudio de una banda transversal-unidad, puede realizarse como el de una viga continua. Al hacer esto, y puesto que la deflexión de dos placas adyacentes puede no ser la misma, es necesario considerar la viga continua como apoyada sobre unos soportes elásticos, como aparece gráficamente en la figura 2, con los apoyos dibujados como muelles.

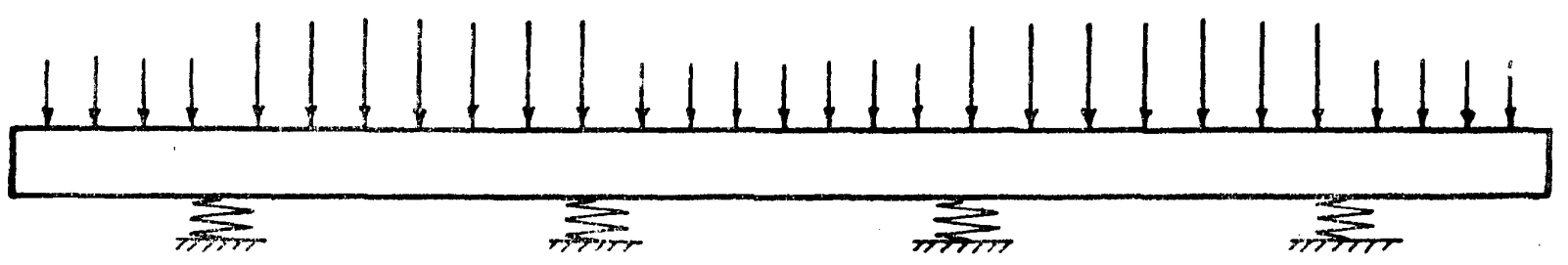

En el sistema estructural, que aparece simbólicamente en la figura 2, el momento sobre un soporte cualquiera puede ser expresado como función de los momentos en dos soportes adyacentes, de la carga actuante sobre los dos vanos que están a cada lado del soporte y de la flecha relativa de la viga en el soporte respecto a la flecha de los bordes adyacentes, o, lo que es más conveniente, como una función de la flecha absoluta en los tres soportes mencionados. La expresión dada por el teorema de los tres momentos es un ejemplo clásico de esta relación.

En esta relación, la flecha de la viga en cualquier soporte es, desde luego, igual a la longitud que se acorta o se alarga el muelle. La magnitud de este movimiento puede ser expresada, bien como el producto de la reacción por una constante igual a la carga necesaria para producir un desplazamiento unidad, o, lo que es preferible para este trabajo, como una función de la tensión en el muelle multiplicada por una constante igual al desplazamiento producido por una fuerza unidad. En consecuencia, sobre un soporte cualquiera puede escribirse una ecuación en la cual se relacionan con la carga de la viga los momentos en la misma viga y las tensiones en los muelles de los tres apoyos; esto es, en el soporte que se está considerando y en los dos adyacentes.

En estas ecuaciones, las únicas cantidades difíciles de determinar son las constantes de los muelles. Estos muelles en la viga continua considerada representan la resistencia al desplazamiento de las placas en la estructura al funcionar como placas longitudinales. Por tanto, las constantes de los distintos muelles pueden ser expresadas en función de las características geométricas de las placas; esto es, su longitud, su canto, su espesor, y el ángulo de inclinación de una respecto a la otra o respecto a un plano vertical. Sin embargo, puesto que en un pliegue cualquiera los desplazamientos en dirección del resorte son una función de la interacción de las dos placas que se cortan sobre ese soporte, las constantes de los tres muelles están afectadas no sólo por la geometría de los vanos limitados por estos tres muelles, sino también por la de los dos vanos adyacentes. Por consiguiente, en un punto cualquiera influye la geometría de cuatro vanos. 
Al expresar las constantes de los muelles en función de las propiedades geométricas y físicas de las placas se supone, tácitamente, que estos factores están, a su vez, relacionados con las tensiones longitudinales de las placas. Si las tensiones de las placas fueran solamente producidas por la flexión, los tres esfuerzos en los pliegues correspondientes a los tres puntos de apoyo serían suficientes para tener en cuenta el desplazamiento de las cuatro placas. Pero, puesto que el esfuerzo tangencial transversal, $s$, y por tanto, el longitudinal, no necesitan ser cero en una cualquiera de las extremidades del elemento aislado de la figura 1c, cada placa, considerada individualmente, está sometida a tensiones tanto axiles como producidas por las flexiones. Esto hace necesario el relacionar el desplazamiento de una placa con las tensiones en las fibras que estén a ambos lados de la línea de centros de gravedad. Como consecuencia de esto, la tensión longitudinal en el pliegue que está alejado dos tramos del pliegue que se considera, o en otras palabras, en el borde exterior de las dos placas exteriores, ha de incluirse como una incógnita si la acción del muelle ha de ser formulada correctamente. De aquí, puede generalizarse que en un pliegue cualquiera de una estructura en placa plegada, es posible establecer una relación entre los momentos de tres pliegues y las tensiones longitudinales en cinco pliegues y, además, la carga exterior que actúa sobre los dos pliegues adyacentes.

De lo anteriormente dicho pueden sacarse dos conclusiones. Primera, si la flexión relativa de un pliegue a su adyacente es pequeña, entonces los momentos transversales en las placas plegadas pueden calcularse considerando una banda transversal unidad de la placa plegada como una viga continua rígidamente apoyada en los pliegues. Esta condición se cumple, bastante aproximadamente, cuando un cierto número de placas idénticas o de combinaciones idénticas de placas se repiten en dirección transversal y están sujetas al mismo tipo de cargas.

El segundo hecho que aparece, es que la diferencia entre el momento transversal obtenido así y el obtenido para una viga continua sobre soportes rígidos es función de la rigidez vertical de las placas. Si las placas son flexibles en la dirección longitudinal, esto es, cuando el canto proyección vertical de las placas es pequeño comparado con la luz longitudinal-por ejemplo, menor de un quinceavo del vano-, puede apreciarse un notable incremento del momento transversal en las zonas próximas a los bordes libres. Con ciertas configuraciones es posible obtener un momento transversal, en algunos de los pliegues cercanos al borde libre, mayor que el momento estático de la carga respecto al pliegue. Por esta razón, en algunos casos es de desear un aumento en el canto de las placas, no sólo por la consideración de su efecto en la cantidad de armadura longitudinal necesaria, sino también por su efecto en la resistencia transversal.

En la discusión anterior se ha insistido en el comportamiento de una placa típica transversal con el efecto de las acciones longitudinales introducidas sólo indirectamente.

Esto es debido, principalmente, al hecho de que, con mucha frecuencia, la magnitud de los momentos transversales fija o el espesor de la placa o la anchura de cada pliegue. Por esto, es esencial para un buen proyecto el entendimiento de los factores que controlan la magnitud del momento, especialmente cerca de los bordes libres. No obstante, tiene el mismo interés para tener un panorama completo del comportamiento en dirección longitudinal. Afortunadamente, aunque algo más complicado, el mismo tipo de estudio cualitativo que se hace para la acción transversal puede aplicarse a la acción longitudinal.

Con este objeto se examina, como elemento aislado, una banda longitudinal formada por dos placas adyacentes. Esto difiere del procedimiento previamente empleado en que la banda considerada era de ancho unidad. Se puede iniciar con una banda formada por una placa individual. Sin embargo, para la exposición, nada se gana empezando con el equilibrio de fuerzas de un elemento aislado. Consideremos, por lo tanto, dos placas como un elemento unitario aislado, tal como aparece en la figura 3. Esta porción de la estructura total está sometida no sólo a las cargas verticales exteriores, sino también a fuerzas a lo largo de sus bordes que representan las reacciones de las placas adyacentes. Aunque la sección transversal del elemento aislado no es de una forma convencional, sin embargo las tensiones longitudinales pueden obtenerse por las fórmulas convencionales procedentes de la flexión. Pero en este caso, y porque la dirección de las cargas no es necesariamente coplanaria con las placas o pasando por el centro de esfuerzos tangenciales, las tensiones en un punto creadas por el momento y por el esfuerzo axil deben combinarse con el efecto de rotación a lo largo del eje longitudinal. El trabajo que 
esto representa no es tan sencillo como pudiera suponerse $y$, consecuentemente, no se le recomienda como procedimiento de proyecto.

Expresando las tensiones en el centro del vano, en el pliegue, y en los bordes libres del elemento que aparece en la figura 3, en función de las cargas conocidas y de las fuerzas de borde incógnitas $\mathrm{S}_{1}, \mathrm{~S}_{2}, \mathrm{~N}_{1}$ y $\mathrm{N}_{2}$, se obtienen tres ecuaciones simultáneas. El momento transversal que actúa a lo largo del borde no aparecerá en estas ecuaciones, porque las tensiones longitudinales están referidas solamente a momentos perpendiculares al plano del momento transversal. Por eliminaciones sucesivas de $S_{1}$ y $S_{2}$, las tres ecuaciones pueden reducirse a una ecuación única en la cual las tensiones en los tres puntos están relacionadas con la carga exte-

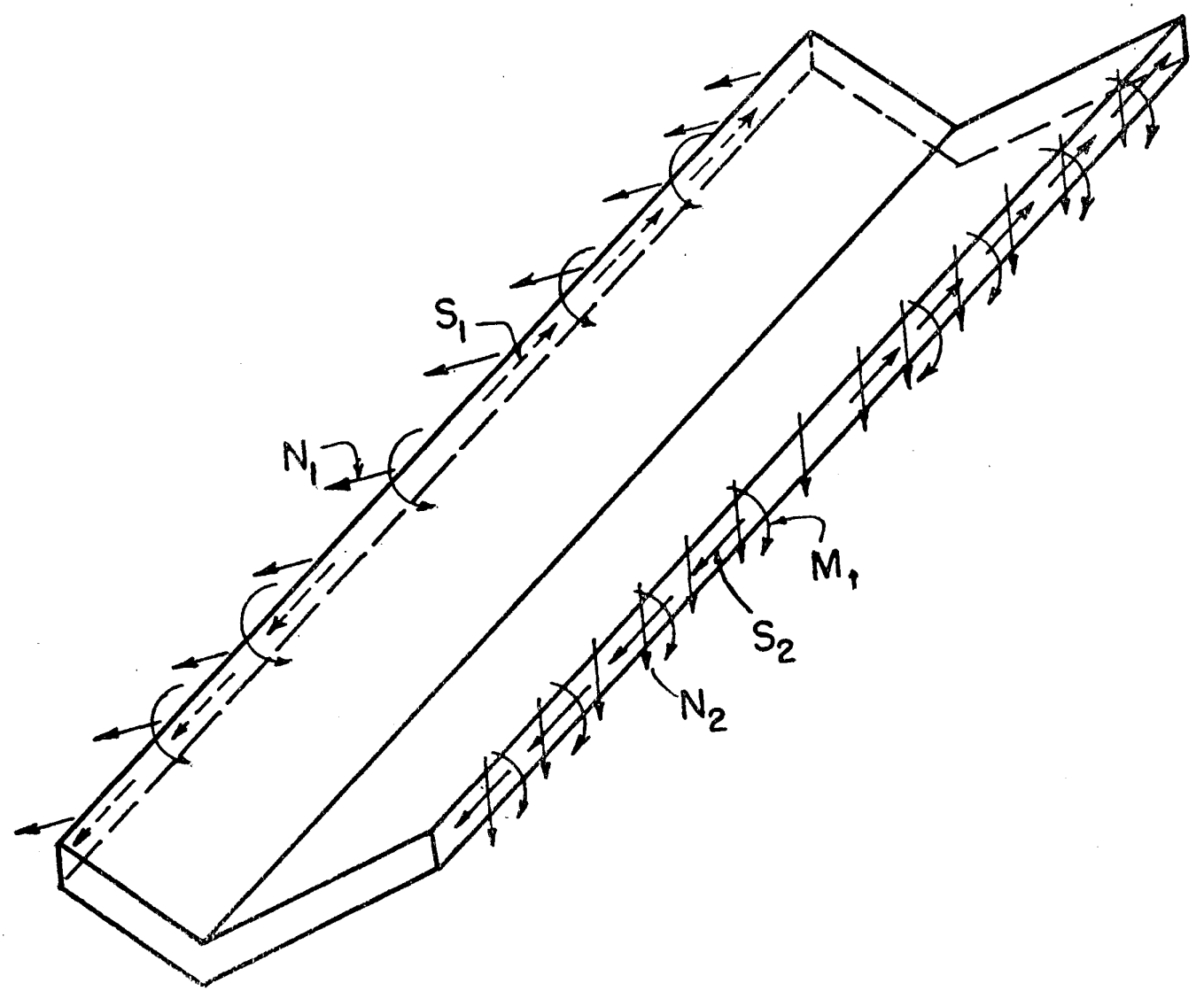

rior sobre las dos placas y con las fuerzas de borde incógnitas $N_{1}$ y $N_{2}$. Una consideración del equilibrio de fuerzas en la dirección transversal, tal como se hace detalladamente en el apéndice, demuestra que las fuerzas $\mathrm{N}$ en un borde son función, exclusivamente, de la carga sobre placas adyacentes a dicho borde; de la diferencia entre los valores de los momentos transversales en el borde y en sus bordes adyacentes, y de la geometría de las placas. En consecuencia, la sustitución de las fuerzas de borde $\mathrm{N}_{1}$ y $\mathrm{N}_{\mathrm{s}}$ por sus equivalentes en la ecuación anterior, se traduce en una expresión en la que las tensiones en tres puntos adyacentes y el momento en cinco puntos están relacionadas con la carga exterior en cuatro placas.

Considerando todo lo anteriormente dicho, aparecen, como evidentes, varios hechos que merecen una ligera atención y cuya consideración puede ayudar para impedir cálculos inútiles. $\mathrm{Si}$ los momentos transversales son todos iguales, como, por ejemplo, en los pliegues interiores 
de las placas plegadas en forma de $\mathrm{V}$ con mucho canto, o pueden obtenerse por consideraciones estáticas, las tensiones longitudinales pueden determinarse directamente a partir de las cargas exteriores exclusivamente. Como corolario se sigue que, si además las tensiones longitudinales son nulas en los dos pliegues de una placa, las tensiones pueden obtenerse por la teoría corriente de flexión.

En resumen: es preciso el estudio de la placa plegada, principalmente para determinar el efecto de las distorsiones de borde. Los bordes libres y las placas exteriores tienden a flectar más de lo que se obtiene al considerar la placa como una viga ordinaria. Debido a esto, la intensidad de la tensión longitudinal máxima en las placas exteriores puede ser mayor, o ser la distribución de tensiones enteramente distinta de la que se anticipa por un análisis convencional por el método de la viga. Sin embargo, la suma de fuerzas de compresión y de tracción continuarán siendo aproximadamente las mismas. De aquí que el valor del análisis de la placa plegada reside no tanto en una determinación exacta de la capacidad resistente, sino, principalmente, en que asegura un comportamiento satisfactorio bajo las cargas de servicio y conduce a una mejor evaluación del momento transversal.

\section{Análisis}

\section{Placas plegadas simplemente apoyadas}

El principal problema asociado con el análisis de las placas plegadas es el de hacer compatibles los dos desplazamientos: el obtenido a partir de la resistencia longitudinal con el obtenido de la colaboración transversal. En un sentido estricto, esta igualdad de desplazamientos debe cumplirse no solamente en unos cuantos puntos, por ejemplo, a lo largo de una banda típica, sino que debe cumplirse en todos los puntos de la superficie. Sin embargo, en muchos casos, y especialmente en las placas plegadas, es posible obtener valores muy razonables obligando a cumplir esta condición de compatibilidad de desplazamientos solamente en el centro del vano. Esta aproximación, aunque es aceptable para determinar las tensiones y los momentos críticos, tiende a enmascarar la distribución real de tensiones. Para evitar esto, y al mismo tiempo, suministrar una sólida base para su ulterior desarrollo, el procedimiento utilizado en este artículo se funda en la compatibilidad de desplazamientos en todos los puntos de la lámina. Para asegurar esta igualdad es necesario expresar la carga uniforme como suma de cargas sinusoidales, correspondiendo, cada una de estas cargas, a un término de la serie de Fourier, cuya expresión es:

$$
w=\frac{4 w}{\pi}\left(\operatorname{sen} \frac{\pi x}{L}+\frac{1}{3} \operatorname{sen} \frac{3 \pi x}{L}+\frac{1}{5} \operatorname{sen} \frac{5 \pi x}{L}\right)
$$

La distribución de estas cargas aparece, gráficamente, en la figura 4. En realidad, para las necesidades de proyecto sólo se utiliza la primera carga parcial. El efecto de las sucesivas cargas parciales se notará, principalmente, en zonas próximas a los apoyos, y no producirá ninguna tensión importante en el centro de cada vano. Más aún, en la mayor parte de los casos prácticos el uso de cargas parciales sinusoidales no presenta ninguna dificultad, puesto que los momentos transversales para la segunda carga parcial y las sucesivas pueden hallarse, sin necesidad de recurrir a un complicado estudio de placa plegada, tratando una banda transversal como viga continua sobre apoyos fijos. Esta simplificación es posible porque cada una de las cargas parciales puede ser considerada separadamente como una carga que actúa sobre una placa plegada cuya luz sea la mitad de la longitud de onda de la carga sinusoidal que se esté considerando. Con esta reducción en la luz efectiva de proyecto, la placa es tan rígida en sentido longitudinal que, para todos los efectos prácticos, cada placa puede considerarse apoyada en los pliegues. 

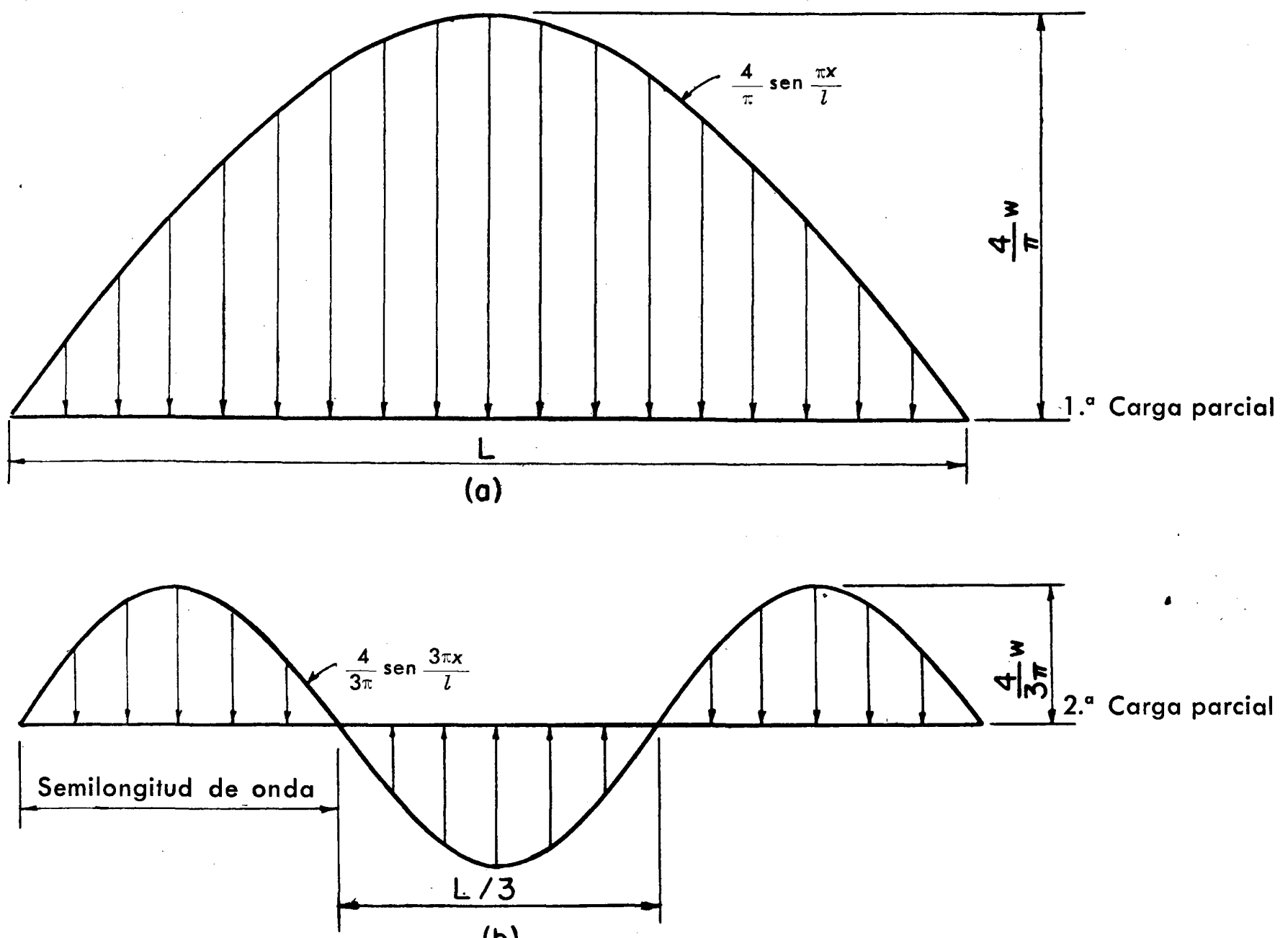

(b)

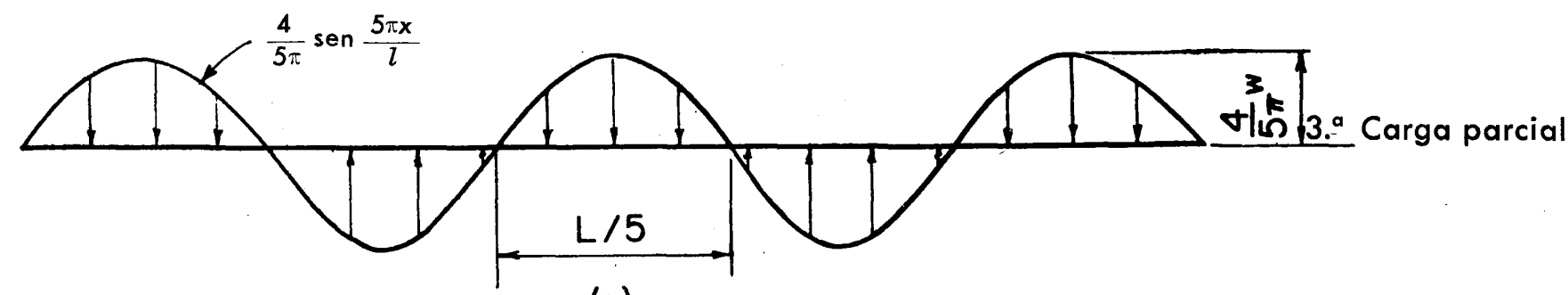

(c)

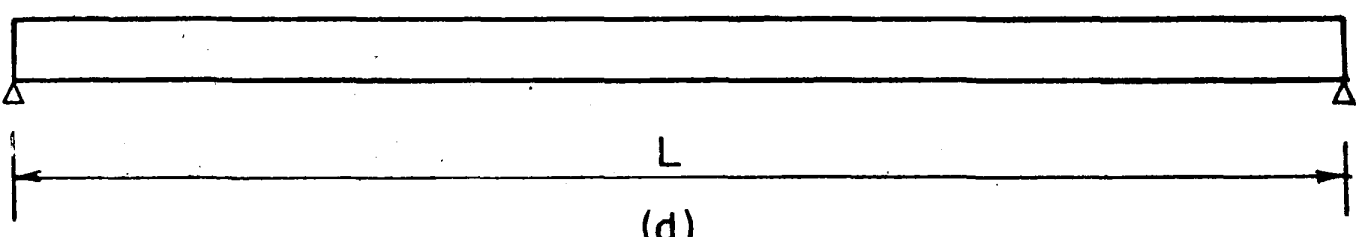

(d) 
Otra ventaja de este procedimiento es que, al satisfacer la compatibilidad en el centro del vano, automáticamente se satisface en todas las demás secciones. En consecuencỉa, se centrará la atención únicamente en las relaciones entre fuerzas en el centro del vano de una placa plegada simplemente apoyada, teniendo en cuenta que los momentos y las tensiones en otra sección cualquiera variarán de acuerdo con la ley sen $(n \pi x / \mathrm{L})$.

En la discusión que antecede se ha señalado indirectamente que, si se conocen el momento transversal y las tensiones longitudinales en cada pliegue, se pueden obtener el momento

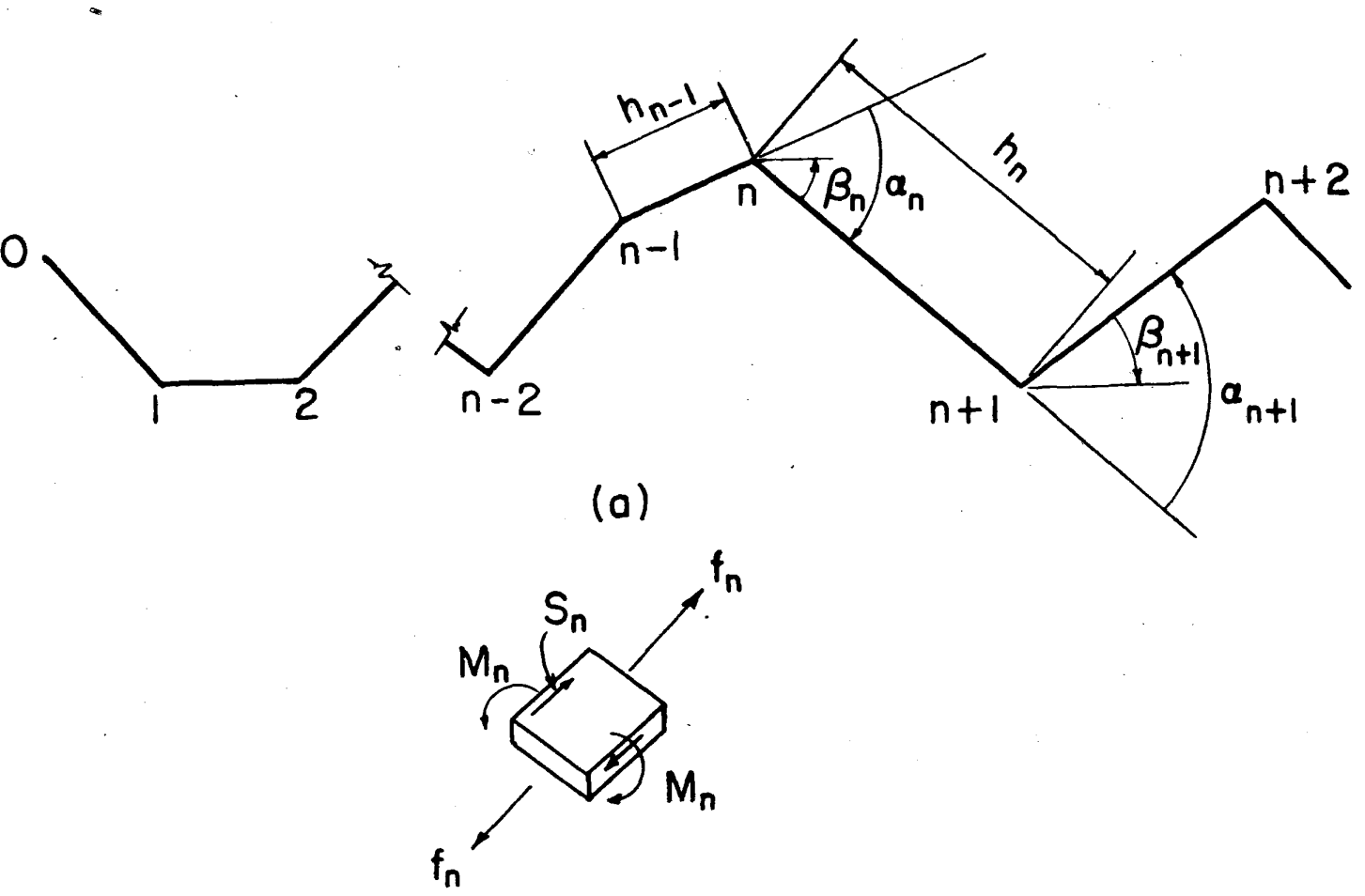

(b)

y la tensión en otro punto cualquiera por medio de la estática o por la teoría de flexión ordinaria. Por tanto, si la figura 5a representa una banda transversal en el centro del vano de una placa plegada simplemente apoyada de forma cualquiera, es preciso solamente determinar las tensiones y los momentos en los distintos pliegues. Como se ve a mayor escala en la figura $5 \mathrm{~b}$, un elemento de un pliegue cualquiera, designado con el subíndice $n$, está sometido a un esfuerzo longitudinal, $f_{n} ;$ a un esfuerzo cortante, $\mathrm{S}_{n}, \mathrm{y}$ a un momento transversal, $\mathrm{M}_{n}$. Como es posible calcular la tensión tangencial $\mathrm{S}_{n}$ a partir de las tensiones longitudinales, en realidad quedan únicamente dos incógnitas por pliegue. Vemos que son suficientes para obtener una solución dos ecuaciones en cada uno de los pliegues, ecuaciones que han de relacionar entre sí las tensiones, los momentos y las cargas existentes. Las dos ecuaciones, que se obtienen 
detalladamente en el Apéndice, se basan en satisfacer la condición de continuidad en dirección transversal, y en la estática de cada pliegue. La primera, que se considera obtenida a partir de la condición de compatibilidad de deformaciones en el pliegue $n$, puede escribirse:

$$
\begin{aligned}
& 2 \frac{h_{n-1}}{h_{n}}\left(\frac{t_{n}}{t_{n-1}}\right) M_{n-1}+4\left[1+\frac{h_{n-1}}{h_{n}}\left(\frac{t_{n}}{t_{n-1}}\right)^{3}\right] M_{n}+2 M_{n+1} \\
- & \left(\frac{L}{\pi}\right)^{2}\left(\frac{t_{n}}{h_{n}}\right)^{3}\left[C_{n}^{n-2} f_{n-2}-C_{n}^{n-1} f_{n-1}+C_{n}^{n} f_{n}-C_{n}^{n+1} f_{n+1}+C_{n}^{n+2} f_{n+2}\right] \\
= & -h_{n}\left[\left(\frac{h_{n-1}}{h_{n}}\right)^{2}\left(\frac{t_{n}}{t_{n-1}}\right)^{3} \frac{W_{n-1} \cos \beta_{n-1}}{2}+\frac{W_{n} \cos \beta_{n}}{2}\right]
\end{aligned}
$$

en la cual los símbolos utilizados representan:

$\mathrm{M}=$ momento flector transversal en un pliegue. Se le considera positivo cuando produce tracción en la cara inferior de la placa.

$f=$ tensión longitudinal. Positiva cuando es de compresión.

$f_{p}=$ tensión longitudinal producida por el pretensado de las placas consideradas aisladas. El primer subíndice indica la posición de la tensión, y el subíndice combinado señala los pliegues adyacentes a la placa.

$\mathrm{W}=$ carga vertical total que actúa sobre la placa. Positiva cuando actúa hacia abajo.

$h=$ longitud de cada placa.

$t=$ espesor de la placa.

$\beta=$ ángulo que forma la placa con la horizontal. Positivo en el sentido de las agujas del reloj y a partir de la placa.

$\alpha=$ ángulo formado por la prolongación de una placa con la adyacente. Positivo en el sentido de las agujas del reloj y a partir de la prolongación de la placa.

$$
\begin{aligned}
& c_{n}^{n-2}=\frac{n_{n}^{2}}{n_{n-2} h_{n-1} \operatorname{sen} a_{n-1}} \\
& c_{n}^{n-1}=\frac{h_{n}^{2}}{n_{n-2} h_{n-1} \operatorname{sen} a_{n-1}}+\left(\frac{h_{n}}{h_{n-1}}\right)^{2}\left(\operatorname{ctg} a_{n-1}+\operatorname{ctg} a_{n}\right)+\frac{h_{n}}{h_{n-1} \operatorname{sen} a_{n}} \\
& \left.c_{n}^{n}=\left(\frac{h_{n}}{n_{n-1}}\right)^{2} \operatorname{ctg} a_{n-1}+\operatorname{ctg} a_{n}\right)+\operatorname{ctg} a_{n}+\operatorname{ctg} a_{n+1}+\frac{2 h_{n}}{n_{n-1} \operatorname{sen} a_{n}} \\
& c_{n}^{n+1}=\frac{h_{n}}{n_{n-1} \operatorname{sen} a_{n}}+\operatorname{ctg} a_{n}+\operatorname{ctg} a_{n+1}+\frac{n_{n}}{n_{n+1} \operatorname{sen} a_{n+1}} \\
& c_{n}^{n+2}=\frac{n_{n}}{n_{n+1} \operatorname{sen} a_{n+1}}
\end{aligned}
$$


El subíndice utilizado con los símbolos indica la tensión, momento, ángulo, placa o pliegue, a que se refiere. La segunda ecuación en el pliegue, $n$, se obtiene, fundamentalmente, de consideraciones estáticas:

$$
\begin{aligned}
& \frac{n_{n-1} t_{n-1}}{h_{n} t_{n}} f_{n-1}+2\left(1+\frac{h_{n-1} t_{n-1}}{h_{n} t_{n}}\right) f_{n}+f_{n+1} \\
& +\frac{6}{f_{n} h_{n}}\left(\frac{L}{\pi h_{n}}\right)^{2}\left[C_{n}^{n-2} M_{n-2}-C_{n}^{n-1} M_{n-1}+C_{n}^{n} M_{n}-C_{n}^{n+1} M_{n+1}+C_{n}^{n+2} M_{n+2}\right] \\
& =\frac{3}{t_{n}}\left(\frac{L}{\pi n_{n}}\right)^{2}\left[\left(W_{n-2}+W_{n-1}\right) \frac{h_{n} \cos \beta_{n-2}}{h_{n-1} \operatorname{sen} a_{n-1}}-\left(W_{n-1}+W_{n}\right)\left(\frac{\cos \beta_{n-1}}{\operatorname{sen} a_{n}}+\frac{n_{n} \cos \beta_{n}}{n_{n-1} \operatorname{sen} a_{n}}\right)\right. \\
& \left.\quad+\left(W_{n}+W_{n+1}\right) \frac{\cos \beta_{n+1}^{\vdots}}{\operatorname{sen} a_{n+1}}\right] \\
& +\left(\frac{h_{n-1} t_{n-1}}{n_{n}^{\dagger} n}\right)\left(2 f_{n, n-1}^{p}+f_{n-1, n}^{p}\right)+2 f_{n, n+1}^{p}+f_{n+1, n}^{p}
\end{aligned}
$$

Las dos ecuaciones anteriores son válidas en cada uno de los pliegues, excepto en los pliegues números $0 \mathrm{v} 1$. En estos puntos el momento ha de determinarse por consideraciones estáticas, y solamente son incógnitas las tensiones longitudinales. De aquí, que se precise una sola ecuación en cada uno de estos puntos. Para el punto 0 tenemos:

$$
\begin{aligned}
& 2 f_{0}+f_{1}+\frac{6}{t_{0} h_{0}}\left(\frac{L}{h_{0} \pi}\right)^{2} \frac{h_{0}}{h_{1} \operatorname{sen} a_{1}} M_{2} \\
& =-\frac{3}{t_{0}}\left(\frac{L}{h_{0} \pi}\right)^{2}\left(2 W_{0}+W_{1}+W_{0} \frac{h_{0} \cos \beta_{0} \cos \beta_{1}}{h_{1}} \frac{\cos \beta_{1}}{\operatorname{sen} a_{1}}+2 f_{01}^{p}+f_{10}^{p}\right.
\end{aligned}
$$

y para el pliegue 1, la relación es:

$$
\begin{aligned}
& \frac{n_{0} t_{0} f_{0}}{h_{1} t_{1}}+2\left(1+\frac{h_{0} t_{0}}{n_{1} t_{1}}\right) f_{1}+f_{2}-\frac{6}{n_{1} t_{1}}\left(\frac{L}{n_{1} \pi}\right)^{2}\left(c_{1}^{2} M_{2}-c_{1}^{3} M_{3}\right) \\
= & \frac{3}{f_{1}}\left(\frac{L}{\pi h_{1}}\right)^{2}\left[\left(2 w_{0}+w_{1}\right)\left(\frac{\cos \beta_{0}}{\operatorname{sen} a_{1}}+\frac{n_{1} \cos \beta_{1}}{n_{0} \operatorname{sen} a_{1}}\right)-\left(w_{1}+w_{2}\right) \frac{\cos \beta_{2}}{\operatorname{sen} a_{2}}+w_{0} \frac{h_{0}}{n_{1}} \cos \beta_{0} C_{1}\right] \\
& +2 f_{12}^{p}+f_{21}^{p}+\frac{h_{0} t_{0}}{h_{1} p_{1}}\left(2 f_{10}^{p}+f_{01}^{p}\right)
\end{aligned}
$$

en la cual:

$$
\begin{aligned}
& c_{1}=\left(\operatorname{ctg} a_{1}+\operatorname{ctg} a_{2}\right)+\frac{h_{1}}{h_{0} \operatorname{sen} a_{1}} \\
& c_{1}^{2}=\operatorname{ctg} a_{1}+\operatorname{ctg} a_{2}+\frac{h_{1}}{h_{2} \operatorname{sen} a_{2}}+\frac{n_{1}}{h_{0} \operatorname{sen} a_{1}} \\
& c_{1}^{3}=\frac{h_{1}}{h_{2} \operatorname{sen} a_{2}}
\end{aligned}
$$

Con dos ecuaciones por pliegue, excepto para el primero, en el cual sólo hay una, y exceptuado también el borde exterior, el número de ecuaciones es igual a dos veces el número de placas menos dos. En muchos casos este número puede reducirse a la mitad por existir simetría o anti- 
metría. Para ilustrar el uso de estas ecuaciones se estudiará el sencillo ejemplo que aparece en el esquema de la tabla 1. Para evitar errores se recomienda una sistematización de los cálculos tal como se indica en esa tabla 1 .

\section{Ejemplo}

' En la tabla 1 aparecen detalladas las características geométricas y trigonométricas de la placa plegada que se dibuja en su parte superior. Los cálculos necesarios para determinar las distintas constantes de las ecuaciones se indican en las columnas 10 a 23, con la operación de que se trata, definida algebraicamente en la cabecera de la columna correspondiente. Aunque los valores numéricos de esta tabla sólo son aplicables al ejemplo seleccionado, la disposición es utilizable para cualquier otro tipo de placa plegada. Debe hacerse notar que, para muchas columnas, sólo es preciso calcular los valores correspondientes al pliegue específico. En las columnas 24 a 31 se calculan los términos correspondientes a las cargas.

Por conveniencia se repiten las cuatro ecuaciones [8], [16], [20] y [23] en la tabla 2, sustituyendo los términos algebraicos por una designación numérica de la columna correspondiente. Debido a la simetría de carga y a la geometría de este ejemplo, $\mathbf{M}_{1}=\mathrm{M}_{4} ; \mathrm{M}_{2}=\mathrm{M}_{3} ; f_{0}=f_{5}$; $f_{1}=f_{4}$, y $f_{2}=f_{3}$. Por tanto, sólo es preciso escribir las ecuaciones para punto 0 y pliegues 1 y 2, haciendo $n$ igual a 2 en las ecuaciones [8] y [16]. En este ejemplo concretamente, $\mathbf{M}_{n-2}$, $\mathrm{M}_{n-1} \mathrm{y} \mathrm{M}_{n+\infty}$, que corresponden a $\mathrm{M}_{0}, \mathrm{M}_{1}$ y $\mathrm{M}_{4}$, son todos ellos iguales a 0 . Al efectuar las operaciones indicadas, utilizando los valores numéricos apropiados tabulados en la tabla 1 , se obtienen las cuatro ecuaciones simultáneas que figuran en la parte superior de la tabla 2. En este caso, para facilitar la solución de las ecuaciones simultáneas, se ha dividido cada ecuación por el coeficiente $\mathrm{M}_{2}$. Todavía no se ha estudiado exhaustivamente, el problema del número de cifras significativas que se necesitan. Se cree que, en la mayoría de los casos, basta con tres cifras significativas. Sin embargo, fueron empleadas cuatro cifras significativas porque con máquina de cálculo resulta igual de fácil el uso de tres que el de cuatro cifras.

Cuando el número de ecuaciones es pequeño, digamos de menos de ocho, es posible llevar a cabo una solución directa, en una hora aproximadamente, utilizando algunas de las nuevas técnicas, como el método de Crout, que ha sido especialmente desarrollado para las máquinas de cálculo. Cuando el número de ecuaciones es mayor, es tan grande la posibilidad de error en una solución directa que resulta más factible obtenerla por un proceso de iteración. En varios libros de matemáticas se encuentran descritos algunos de estos procedimientos. El más conocido, y más fácil de dominar, es el procedimiento descrito en la página 227 del libro de Timoshenko: «Theory of Plates and Shells». En resumen, el método consiste en asumir, arbitrariamente, un conjunto de valores para todas las incógnitas menos para la primera. Entonces se multiplican estos valores por el coeficiente numérico apropiado en cada ecuación, y esto para todos los términos que se hallan a la derecha de la línea escalonada. Empezando por la primera ecuación, es posible, al pasar de una ecuación a la siguiente, determinar un nuevo conjunto de valores para las fuerzas desconocidas y los momentos. Estos valores se emplean, ahora, de la misma manera para llegar al conjunto de valores siguientes, repitiéndose el procedimiento hasta que se consigue suficiente convergencia. Se ha llegado a la conclusión de que es más ventajoso para los valores iniciales empezar con $\mathrm{M}_{n}=-w a^{2} / 12$ y $f_{n}=\mathrm{WL}^{2} / 8 s$, donde $s$ es el módulo de sección de una placa o una combinación de placas.

En este ejemplo, las ecuaciones simultáneas fueron resueltas por solución directa y por iteración. Como puede observarse, todos los valores críticos han convergido con un error menor que un $5 \%$ en el cuarto ciclo, aunque el análisis de la misma estructura por la técnica de distribución convencional conduzca a una convergencia extremadamente lenta. Una de las ventajas del procedimiento es que la convergencia puede ser acelerada si, al notar cualquier oscilación de valores, se interpola arbitrariamente entre los valores de los dos conjuntos para el tanteo siguiente.

Los valores indicados en la tabla 1 , multiplicados por $4 / \pi$ para tener en cuenta la diferencia entre una carga uniforme y una sinusoidal, pueden ser considerados como suficientemente exactos. Si se desea una aproximación mayor, se aplica a las otras cargas sinusoidales parciales el mismo procedimiento de conjunto o versiones simplificadas del procedimiento. En este ejem- 
plo se encontró que la segunda carga parcial, variando según la ley sen $3 \pi x / L$ produjo las siguientes tensiones en la parte central del tramo:

$$
\begin{aligned}
& \mathrm{M}_{2}=+234 \text { pies } \cdot \text { libras/pie. } \\
& f_{0}=+8.300 \mathrm{libras} / \mathrm{pie}^{2} \\
& f_{1}=-700 \mathrm{libras} / \mathrm{pie}^{2} \\
& f_{2}=-410 \mathrm{libras} / \mathrm{pie}^{2}
\end{aligned}
$$

Al comparar estos valores con los tabulados en la tabla 2 se ve que la tensión longitudinal producida por esta carga parcial y, del mismo modo, por otras cargas parciales, son o bien un porcentaje pequeño de la producida por la primera carga, o numéricamente insignificantes. La relación entre los momentos producidos por las dos cargas es algo mayor. Sin embargo, es interesante señalar que el momento producido por la segunda carga parcial es casi igual al obtenido, considerando que las placas son continuas con soportes rígidos en cada pliegue.

Combinando la primera y segunda cargas parciales, se obtiene:

$$
\begin{aligned}
& \mathrm{M}_{2}=(4 / \pi)(-1.513+234)=-1.628 \text { pies } \cdot \text { libras } / \text { pie. } \\
& f_{0}=(4 / \pi)(-161.000+8.300)(1 / 144)=-1.350 \text { libras } / \text { pulgada }^{2} . \\
& f_{1}=(4 / \pi)(1.120-700)(1 / 144)=-16 \text { libras } / \text { pulgada }{ }^{2} . \\
& f_{2}=(4 / \pi)(+16.790+410)(1 / 144)=+145 \text { libras } / \text { pulgada }^{2} .
\end{aligned}
$$

Las tensiones tangenciales pueden obtenerse con el valor de los momentos y los esfuerzos obtenidos. Una explicación detallada del procedimiento figura en el apéndice después de la ecuación [33].

\section{Una guía para el proyecto}

Aunque el análisis de una estructura de placa plegada ha sido reducido a operaciones matemáticas, solamente podrá obtenerse una buena comprensión de su comportamiento al comparar los resultados de muchos casos. La tabla 3 ha sido recopilada para suministrar esta comparación $\mathrm{y}$, al mismo tiempo, facilitar el proyecto del tipo más popular de placa plegada. Esta tabla da coeficientes válidos para proyectar los tipos de estructura de placa plegada en $V$, formadas por un número de Uves idénticas con una $V$ exterior asimétrica reforzada por la presencia de una viga de borde vertical. Mientras que el esquema de la tabla 3 indica que los valores han sido calculados basándose en una estructura simétrica respecto al pliegue 6, los coeficientes son aplicables a cubiertas con mayor o menor número de pliegues. En la mayoría de los casos, el efecto del borde libre sólo alcanza al cuarto pliegue. Por tanto, los pliegues más al centro que el cuarto tienen poco efecto sobre el valor de los esfuerzos y momentos de los pliegues exteriores.

Al calcular la tabla 3 se vio que era conveniente alinear los coeficientes de proyecto en función de los cuatro parámetros $h_{v} / h, h_{o} / h, a / d$ y $\mathrm{L}^{2} t / a^{3}$, con los símbolos definidos en el esquema. La selección de estas variables estaba basada en la forma común de definir las estructuras en función de sus dimensiones horizontales y verticales. Más aún, estos parámetros permiten una fácil interpolación de otros valores, además de los incluídos en la lista. El conjunto seleccionado abarca los casos de aplicación práctica y económica. Se han calculado también los coeficientes para valores más grandes de $\mathrm{L}^{2} t / a^{3}$. En la mayoría de ellos, los momentos transversales obtenidos para tales casos indicaban que sería difícil obtener la resistencia necesaria $y$, por esta razón, se han omitido intencionadamente estos valores más altos de $L^{2} t / a^{s}$.

Para no ocupar mucho espacio y evitar repeticiones innecesarias, sólo se dan coeficientes para $M_{2}$ a $M_{5}$ y para la tensión longitudinal de $f_{0}$ a $f_{3}$. El coeficiente del momento en el pliegue 6 o en pliegues más interiores es igual al valor del momento de empotramiento de 0,0833. Los coeficientes de las tensiones longitudinales en pliegues posteriores al 3 son asimismo cons- 
tantes independientes de los parámetros, excepto de la relación $a / d$. Para las relaciones dadas los coeficientes son:

$$
\begin{array}{cccccc}
a / d & \ldots & 2,5 & 2,0 & 1,5 & 1,0 \\
\text { Coeficiente de la tensión } & \cdots & \pm 1,411 & \pm 1,087 & \pm 0,759 & \pm 0,430
\end{array}
$$

En vista de que los coeficientes están dados en forma no dimensional, es importante que todas las unidades sean del mismo tipo. Si w está expresada en libras/pie cuadrado y las dimensiones en pies, el momento calculado y la tensión longitudinal lo estará en pies · libras/pie y libras/pie ${ }^{2}$, respectivamente.

Para ilustrar la facilidad con que pueden obtenerse momentos y tensiones, se calculará a continuación un breve ejemplo. Dada una cubierta en placa plegada en la que

$$
\begin{aligned}
& \mathrm{L}=55 \text { pies } \quad h_{0} / h=0,25 . \\
& a=10 \text { pies } \quad h_{0} / h=0 . \\
& t=0,33 \text { pies } \quad a / d=2,0 . \\
& w=(\text { carga muerta }+ \text { sobrecarga })=86 \text { libras } \times \text { pie }^{2} .
\end{aligned}
$$

entonces:

$$
\mathrm{L}^{2} t / a^{3}=\frac{55^{2} \times 0,333}{10^{3}}=1
$$

y los multiplicadores indicados en la cabeza de la tabla 3 son:

$(4 / \pi) w a^{2}=1,27 \times 86 \times 10^{2}=10.900$ pies $\cdot$ libras $/$ pie.

$$
(4 / \pi) \frac{w \mathrm{~L}^{2}}{a t}=\frac{1,20 \times 86 \times 55^{2}}{10 \times 0,333}=99.400 \mathrm{libras} / \mathrm{pie}^{2} .
$$

Al entrar en la tabla 3, con los parámetros dados, vemos que el coeficiente para $\mathrm{M}_{2}$ es - 0,285. Por tanto, el momento es:

$$
\mathrm{M}_{2}=-0,285 \times 10.900=-3.110 \text { pies-libras } / \text { pie. }
$$

Los otros valores pueden ser determinados de la misma manera. El efecto de la segunda carga parcial puede también ser determinado por medio de la tabla 3. Con este fin se toman la luz y la carga reducidas a la tercera parte del valor dado. Basándose en esto:

$$
\mathrm{L}^{2} t / a^{3}=\frac{(55 / 3)^{2} \times 0,333}{10^{3}}=\frac{1}{9}
$$

y:

$$
w=-36 / 3=-28,7 \mathrm{libras} / \mathrm{pie}^{2} \text { (en la sección central del vano). }
$$

Cuando el valor $\mathrm{L}^{2} t / a^{3}$ es menor que el dado en la tabla 3 como ocurre aquí, puede calcularse el momento suponiendo que los pliegues están rígidamente apoyados, y puede despreciarse la variación de la tensión longitudinal.

La tabla 3 supone que la carga es uniforme en la estructura. Por tanto, se desprecia el efecto del peso del elemento de borde vertical.

\section{Continuidad}

Las ecuaciones anteriores han sido obtenidas basándose en que las placas plegadas están simplemente apoyadas. Un conjunto análogo de ecuaciones, aunque considerablemente más complicado, puede ser establecido para placas plegadas continuas. Esta complejidad es debida, 
principalmente, al hecho de que en las placas plegadas continuas la distribución transversal (no la intensidad) de las tensiones longitudinales no es tan uniforme a lo largo de las placas plegadas como para los vanos simples. En otras palabras, la distribución transversal de las tensiones para cada carga parcial en cualquier sección deja de ser igual a la distribución transversal en la sección central multiplicada por sen $n \pi x / \mathrm{L}$. Han de introducirse otros términos, que son muy complicados en la expresión de las tensiones a fin de cumplir con los requisitos de las coacciones en el extremo.

Una forma expeditiva de superar esta dificultad es relajar la exigencia de satisfacer la condición de compatibilidad de deformaciones a lo largo de toda la unión de dos placas, para satisfacer exclusivamente el requisito de compatibilidad de flechas en el centro del vano. La flecha de cada placa en el centro del vano está determinada como la de una viga continua. Así, por ejemplo, si se supone un empotramiento completo en ambos extremos de la placa, la flecha se toma como $5 \mathrm{PL}^{4} / 384$.

Otro método, que ha sido usado y que ha dado buenos resultados, es el de obtener los esfuerzos longitudinales en el soporte y en el centro del vano, basándose en los momentos creados en una viga cuyos vanos son iguales a los de la placa plegada. En este método, la distribución transversal está basada en un vano efectivo de longitud igual a la distancia entre los puntos de inflexión de la viga continua.

\section{Intersección de tres placas}

Una de las ventajas de este sistema de solución directa es que facilita la determinación de los esfuerzos en estructuras de placa plegada, en las cuales tres placas se cortan en un pliegue como se ve en la figura A8. El mismo método que se emplea para la placa plegada convencional es aplicable a este problema más complejo. Debido al uso, tan poco frecuente, de la disposición de tres placas en el interior de los pliegues, no se han desarrollado para este caso ecuaciones aplicables al proyecto. Sin embargo, para demostrar la forma de obtener tales ecuaciones, en el caso más corriente de tres placas que se cortan en el pliegue exterior, es posible establecer, en los puntos o pliegues a los que afectan, ecuaciones que relacionen las tensiones y los momentos con las cargas exteriores.

La presencia de una placa adicional vertical en el pliegue primero, hace que las ecuaciones [20] y [23], anteriormente dadas para el punto 0 y pliegue 1, no sean válidas. En lugar de éstas deben emplearse las ecuaciones [27] y [29]. Además, al introducir la tercera placa, se introduce otro factor desconocido, que es la tensión longitudinal en la parte inferior de la placa vertical, llamada $f_{v}, y$ debe usarse la ecuación [31]. Esta ecuación, que se obtiene fácilmente al igualar la flecha de cada una de las placas que se cortan entre sí con respecto a la de las demás, expresa la relación entre $f_{v}$ y las tensiones adyacentes. Finalmente, es preciso considerar una pequeña modificación en la ecuación [16] del pliegue 2, como la que aparece en la ecuación [33], con objeto de tener en cuenta el miembro adicional vertical. 


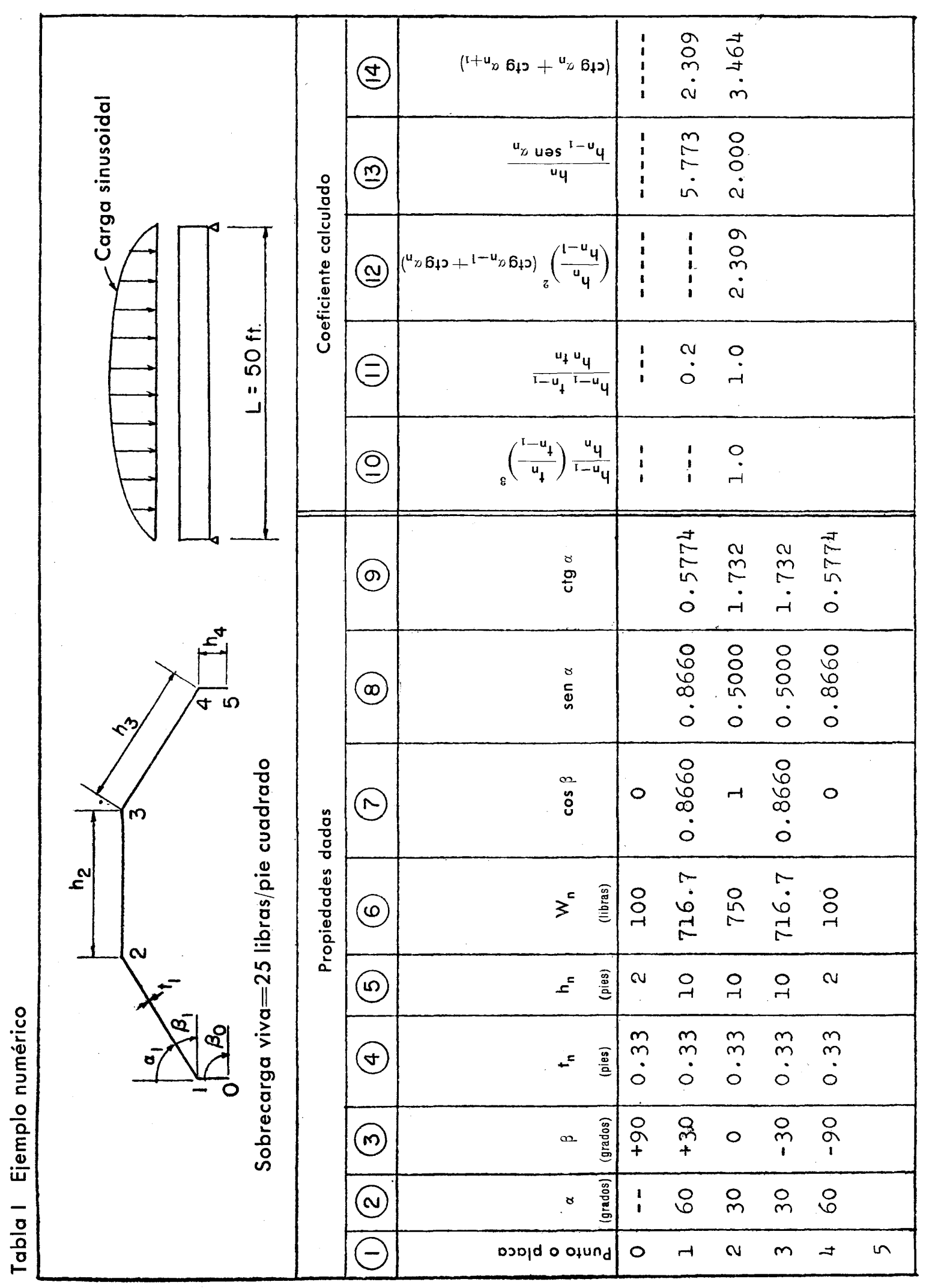




\begin{tabular}{|c|c|c|c|c|}
\hline \multirow{8}{*}{$\begin{array}{l}0 \\
0 \\
0 \\
0 \\
0 \\
0 \\
\vdots \\
0 \\
0 \\
0 \\
\vdots \\
0 \\
0\end{array}$} & (ल) & 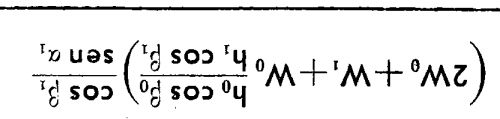 & $\begin{array}{l}\tau \\
\dot{0} \\
-1\end{array}$ & \\
\hline & (8) & ${ }^{0} \delta \operatorname{sos} \frac{{ }^{\frac{1}{4}}}{{ }^{0} y}{ }^{0} M$ & $10 i$ & \\
\hline & (ֻ) & 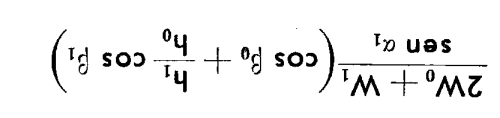 & 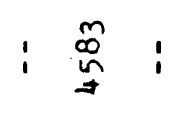 & \\
\hline & ఖ & $\frac{i+u_{p \text { ues }}}{{ }^{i+u_{d} \text { sos }}}\left({ }^{\mathrm{r}+u} M+{ }^{\mathrm{u}} M\right)$ & 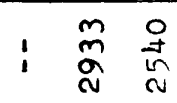 & \\
\hline & (จ) & 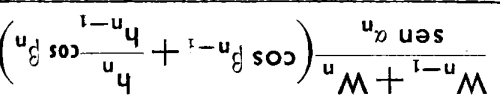 & $i: \frac{*}{\tilde{n}}$ & \\
\hline & (Q) & 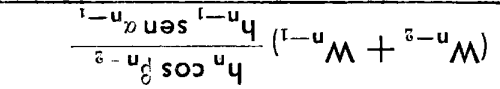 & 110 & \\
\hline & ((2) & $\frac{\tau}{u_{g} \operatorname{sos}{ }^{u} M}$ & $i: \frac{n}{m}$ & \\
\hline & (ब) & $\frac{\tau}{1-u_{\partial j} \operatorname{sos}^{I-u} M}$ & $\begin{array}{l:l} & m \\
: & 0 \\
& m\end{array}$ & \\
\hline \multirow{10}{*}{ 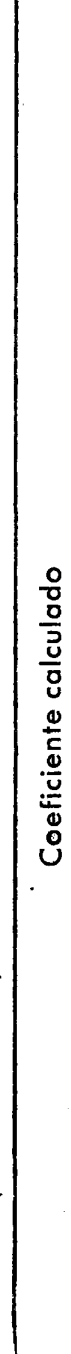 } & (2) & $\left(\frac{l-u_{t}}{u_{t}}\right)_{8}\left(\frac{u_{4}}{1-u_{4}}\right)$ & 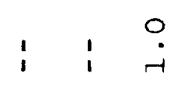 & \\
\hline & (전) & ${ }_{8}\left(\frac{u_{4}^{4}}{u_{1}}\right)_{8}\left(\frac{4}{7}\right)$ & 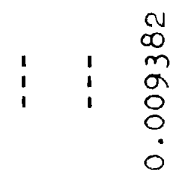 & \\
\hline & (ब) & $\left(\frac{{ }^{4}{ }_{4 x}}{7}\right) \frac{{ }^{4}{ }^{4}{ }^{4}}{9}$ & $\begin{array}{lll}9 & 0 & 8 \\
\dot{a} & i & 0 \\
i & \dot{n} \\
\text { in } & \dot{j} & \dot{\pi}\end{array}$ & \\
\hline & (2) & ${ }_{\varepsilon}\left(\frac{u_{4 x}}{1}\right) \frac{u_{t}}{\varepsilon}$ & $\begin{array}{lll}\sigma & 0 & \circ \\
\dot{\alpha} & \infty & \infty \\
0 & \dot{N} & \dot{N} \\
\text { in } & \stackrel{N}{N} & \stackrel{N}{*}\end{array}$ & \\
\hline & (9) & 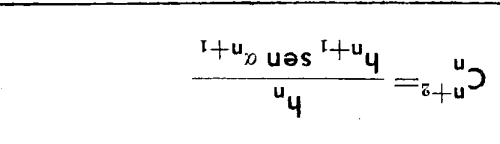 & 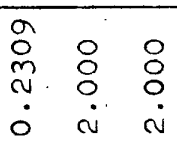 & \\
\hline & $@$ & (61) + (b1) + (21) $={ }_{1+u}^{u}$ & 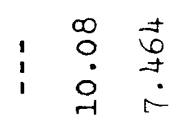 & \\
\hline & $\Theta$ & (11) + (21) $\times 2+$ (21) $={ }_{u}^{u}$ & $\begin{array}{l}: \text { 吉 } \\
\vdots\end{array}$ & \\
\hline & (Q) & (21) + (21) + (91) $={ }_{1-u}^{u}$ & $\begin{array}{lll}1 & \infty \\
1 & 0 \\
1 & 0 \\
1 & \end{array}$ & \\
\hline & (6) & $\frac{1-u_{p} \text { uəs }{ }^{i}-u_{4}{ }^{z-u_{4}}}{u_{4}}={ }^{5}-u^{u^{2}} \supset$ & $i$ & \\
\hline & $\Theta$ & Doold o olund & $H$ & $m \neq$ \\
\hline
\end{tabular}




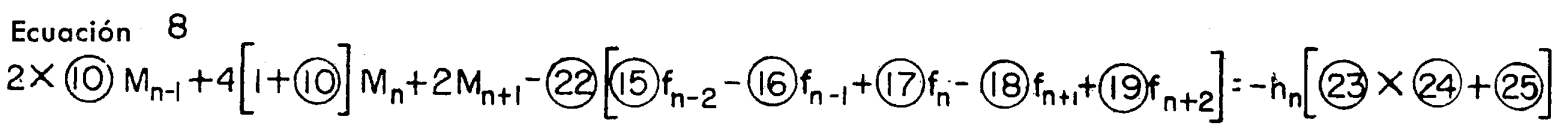

Ecuación 16

(11) $f_{n-1}+2[1+$ (11) $] f_{n}+f_{n+1}+$ (21) $\left[(15) M_{n-2}-(16) M_{n-1}+(17) M_{n}-\right.$ (18) $\left.M_{n+1}+(19) M_{n+2}\right]=-$ (20 $[(26)-(27)+$ (28) $]$

Ecuación 23

(11) $f_{0}+2[1+(11)] f_{1}+f_{2}-(21)\left[(18) m_{2}-(19) M_{3}\right]=$ (20) $\{(29)-(28)+(30[(13)+(14)]\}$

Ecuación 20

$2 f_{6}+f_{1}+$ (21) $\times(19) M_{2}=-(20 \times$ (31)

\begin{tabular}{|c|c|c|c|c|c|c|}
\hline Ecuación n. ${ }^{\circ}$ & $\begin{array}{l}\text { Punto } \\
0 \\
\text { pliegue }\end{array}$ & $\mid \begin{array}{c}M_{2} \\
\text { pie. libra/pie }\end{array}$ & $\begin{array}{c}\boldsymbol{f}_{0} \\
\text { libra/pie cuadrado }\end{array}$ & $\begin{array}{c}f_{1} \\
\text { libra/pie cuadrado }\end{array}$ & $\begin{array}{c}\boldsymbol{f}_{2} \\
\text { libra/pie cuadrado }\end{array}$ & Constante \\
\hline $\begin{array}{r}8 \\
20 \\
23 \\
16\end{array}$ & $\begin{array}{l}2 \\
0 \\
1 \\
2\end{array}$ & $\begin{array}{r}1 \\
1 \\
-1 \\
1\end{array}$ & $\begin{array}{c}-0.005416 \\
+0.01520 \\
+0.005427 \\
0\end{array}$ & $\begin{array}{l}+0.007583 \\
+0.007598 \\
+0.06512 \\
+0.09497\end{array}$ & $\begin{array}{c}-0.002167 \\
0 \\
+0.02713 \\
+0.4749\end{array}$ & $\begin{array}{l}=-685 \\
=-3969 \\
=+1021 \\
=+6351\end{array}$ \\
\hline \multicolumn{2}{|l|}{ Solución directa } & -1513 & $-161,100$ & $-1,129$ & $+16,790$ & \\
\hline 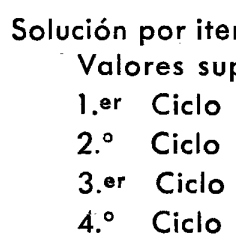 & & $\begin{array}{l}-\quad-785 \\
-1770 \\
-1415 \\
-1603\end{array}$ & $\begin{array}{l}-73,700 \\
-195,000 \\
-148,500 \\
-165,700 \\
-159,200\end{array}$ & $\begin{array}{l}-43,100 \\
+7,600 \\
-4,700 \\
+7,010 \\
-1,900\end{array}$ & $\begin{array}{l}+33,000 \\
+13,300 \\
+18,000 \\
+14,950 \\
+17,130\end{array}$ & \\
\hline
\end{tabular}




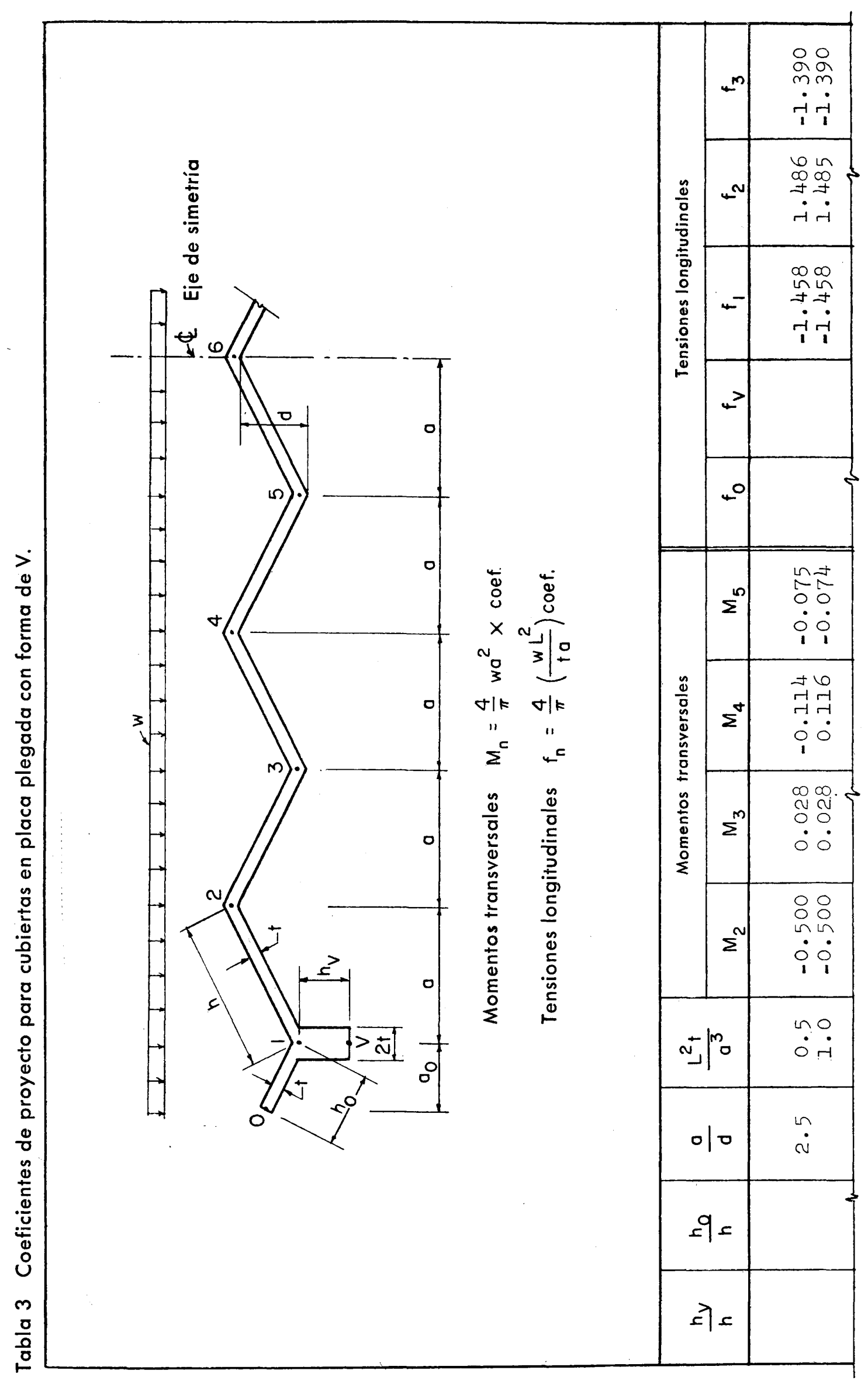




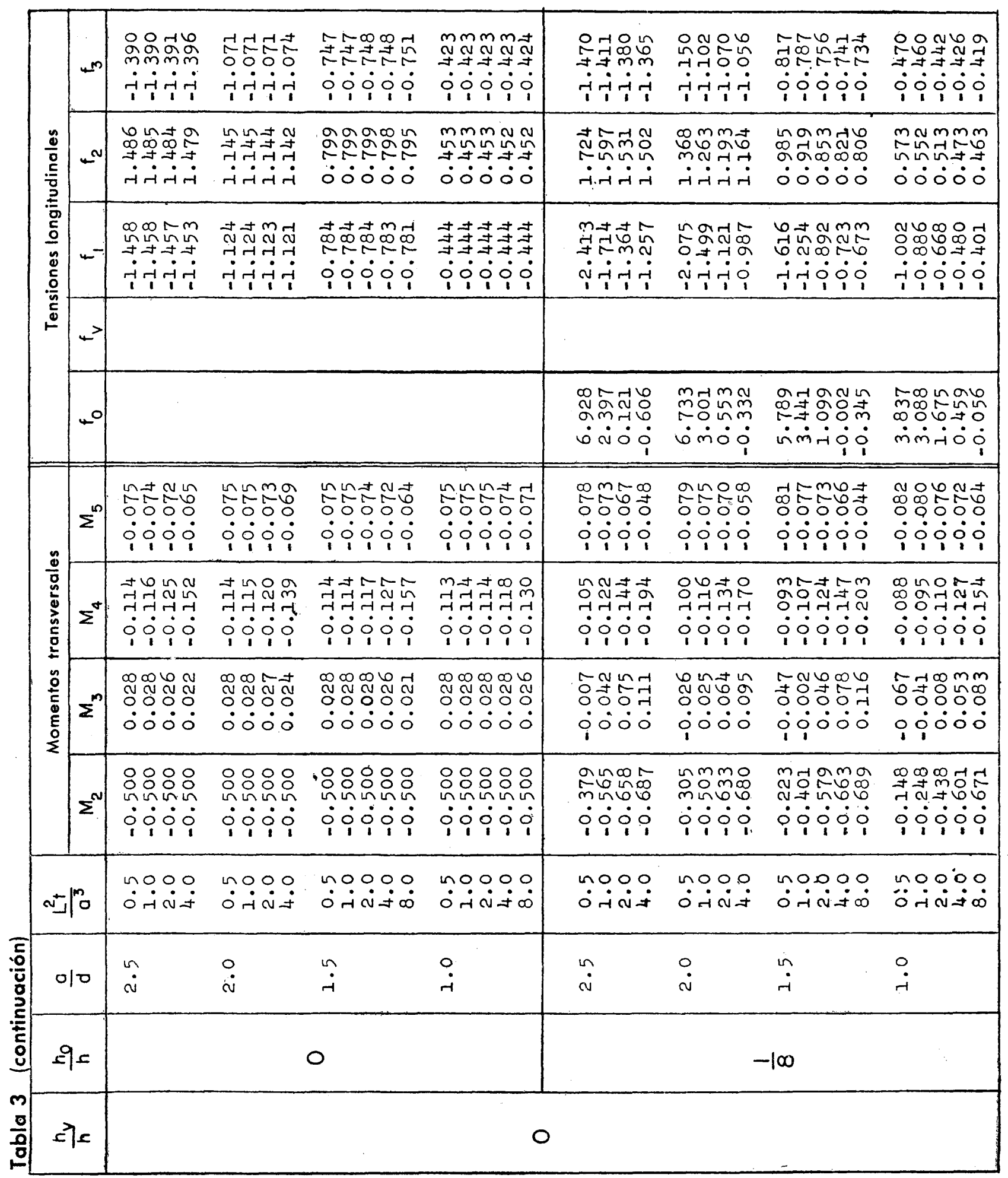




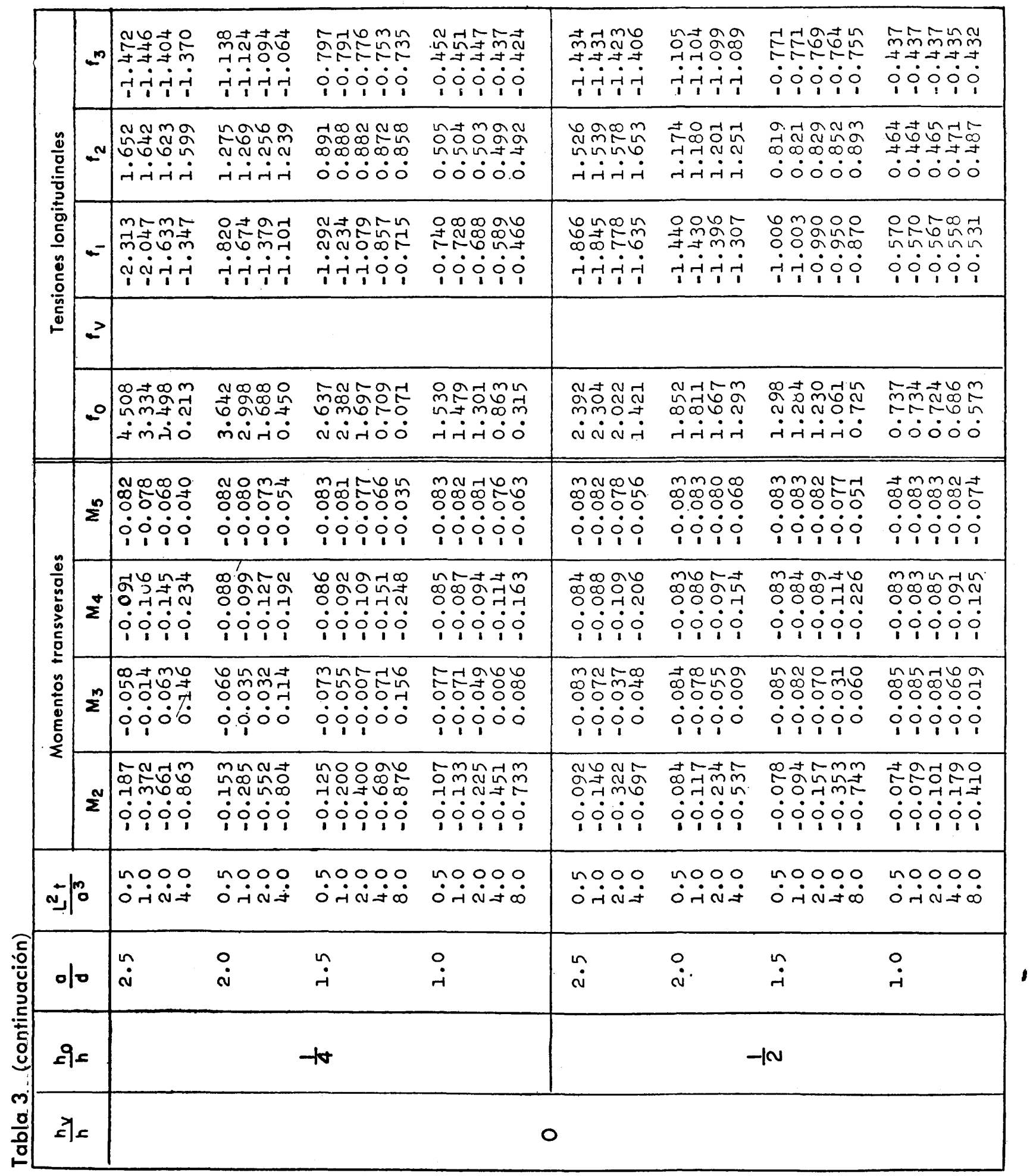




\begin{tabular}{|c|c|c|c|c|c|c|c|c|c|c|}
\hline & & m & 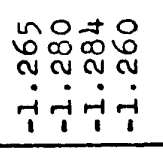 & 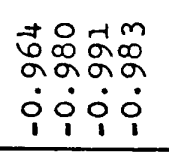 & 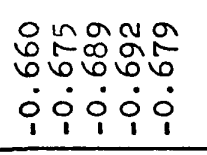 & 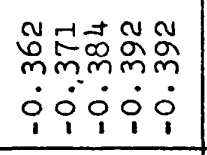 & 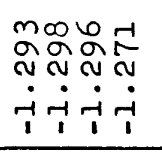 & 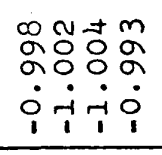 & 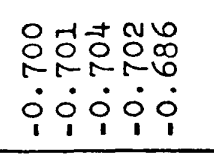 & 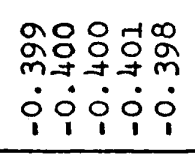 \\
\hline & & N & 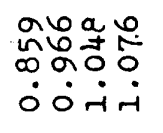 & 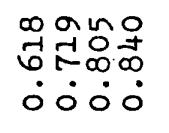 & 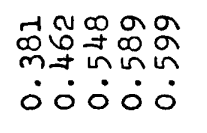 & 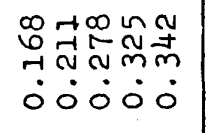 & 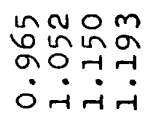 & 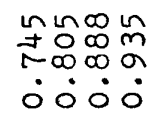 & 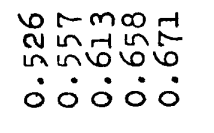 & 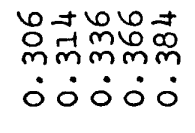 \\
\hline & 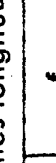 & - & 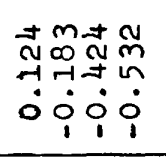 & 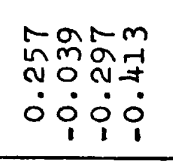 & 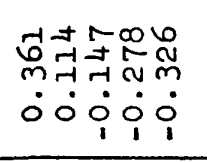 & 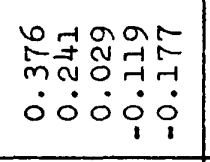 & 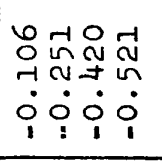 & 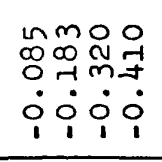 & 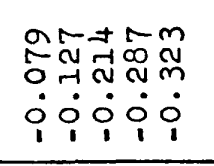 & 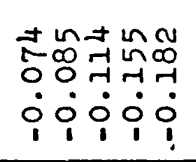 \\
\hline & 柽 & $\geq$ & 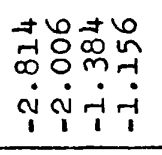 & 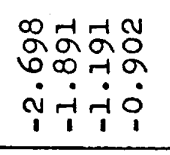 & 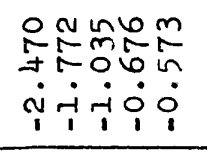 & 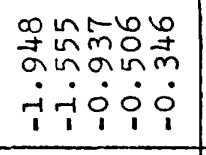 & 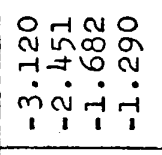 & 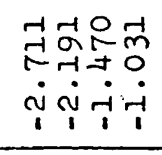 & 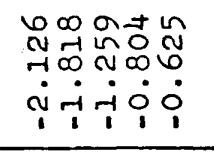 & 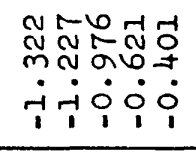 \\
\hline & &. & & & & & 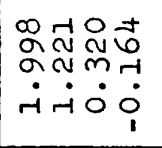 & 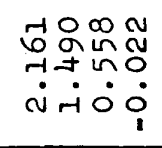 & 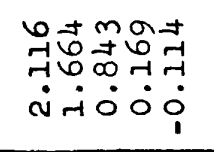 & 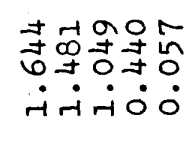 \\
\hline & & $\sum^{\infty}$ & $\begin{array}{l}\text {-19m: } \\
05050 \\
000 \\
000 \\
010 \\
11\end{array}$ & 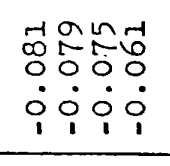 & 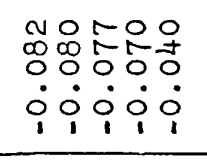 & 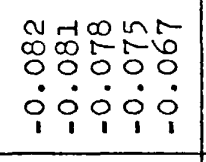 & 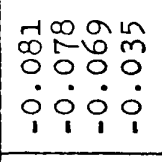 & 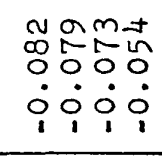 & 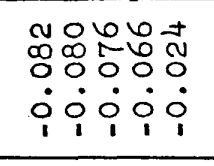 & 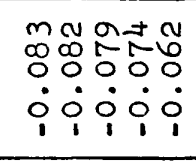 \\
\hline & to & $\Sigma^{+}$ & 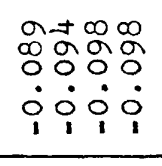 & 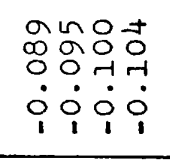 & 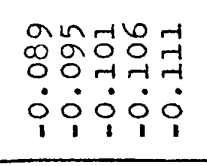 & 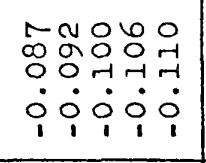 & 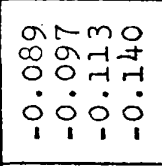 & $\begin{array}{llll}\infty & 0 & 0 & 1 \\
0 & 9 & -1 & m \\
0 & 0 & -1 & -1 \\
0 & 0 & 0 & 0 \\
1 & 0 & 0 & 1\end{array}$ & 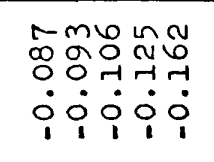 & 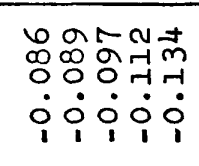 \\
\hline & 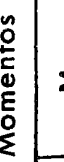 & $\Sigma^{m}$ & 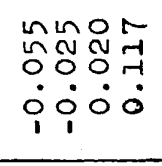 & 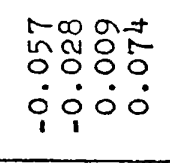 & 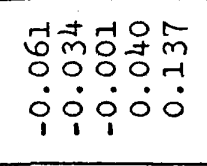 & 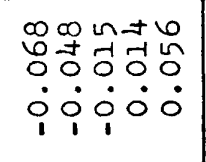 & 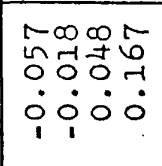 & 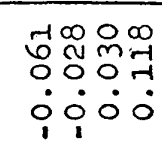 & 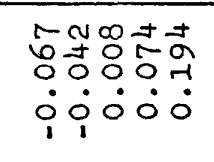 & 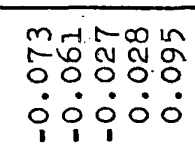 \\
\hline & & $\Sigma^{N}$ & 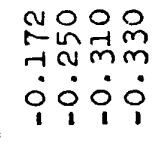 & 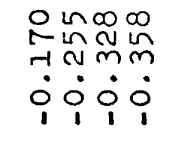 & 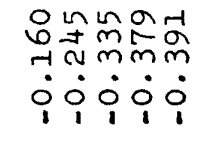 & 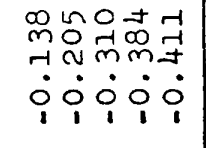 & 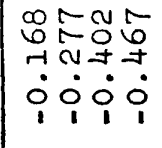 & 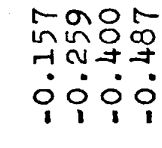 & 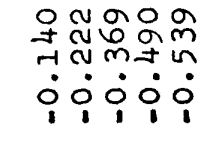 & 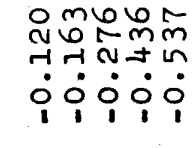 \\
\hline & \multicolumn{2}{|c|}{$N$} & 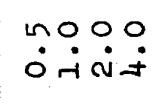 & 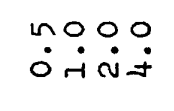 & 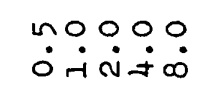 & 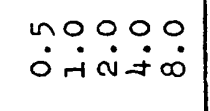 & थ०००० & 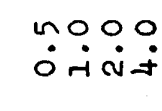 & $\begin{array}{l}n 0000 \\
\dot{0} \\
-i \dot{N}+\infty\end{array}$ & $\begin{array}{l}\text { no: } \\
\dot{\circ} \dot{i} \dot{\sim} \dot{j} \dot{0}\end{array}$ \\
\hline & \multicolumn{2}{|c|}{010} & $\stackrel{\tilde{u}}{\dot{\alpha}}$ & $\stackrel{\circ}{\dot{\sim}}$ & $\stackrel{n}{n}$ & $\stackrel{0}{\circ}$ & $\tilde{\sim}$ & $\begin{array}{l}\stackrel{0}{\sim} \\
\dot{\sim}\end{array}$ & $\stackrel{n}{\sim}$ & $\stackrel{0}{\circ}$ \\
\hline & \multicolumn{2}{|c|}{99} & \multicolumn{4}{|c|}{0} & \multicolumn{4}{|c|}{$-1 \infty$} \\
\hline & \multicolumn{2}{|c|}{ टो } & \multicolumn{8}{|c|}{-100} \\
\hline
\end{tabular}




\begin{tabular}{|c|c|c|c|c|c|c|c|c|}
\hline$\stackrel{m}{*}$ & 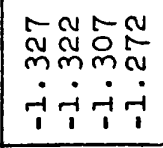 & 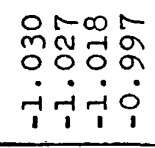 & 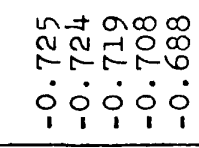 & 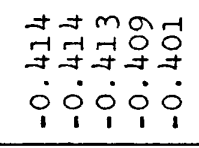 & 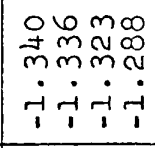 & 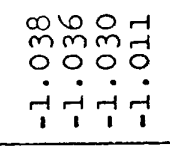 & 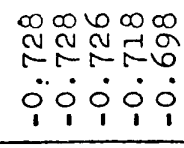 & 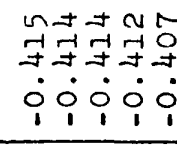 \\
\hline$\cong$ & 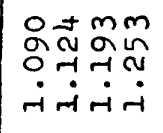 & 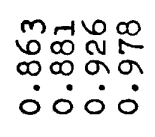 & 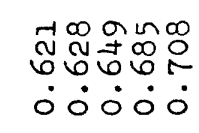 & 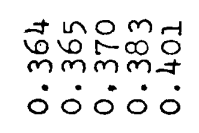 & 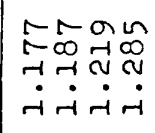 & 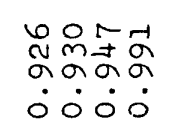 & 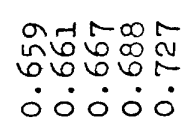 & 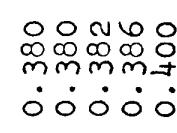 \\
\hline$=$ & 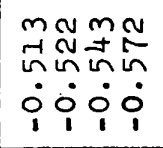 & 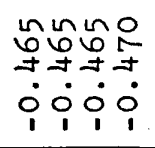 & 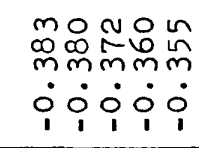 & 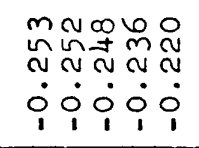 & 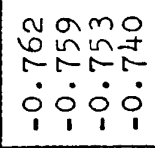 & 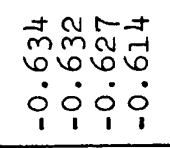 & 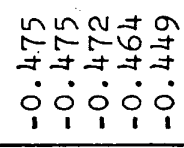 & 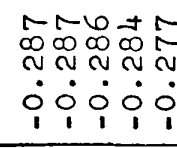 \\
\hline 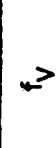 & 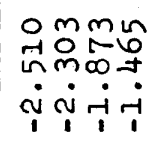 & 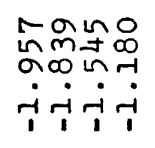 & 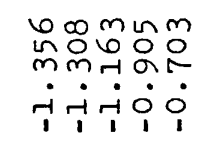 & 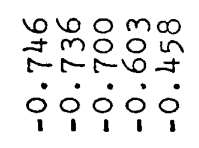 & 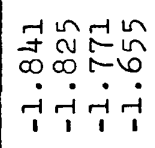 & 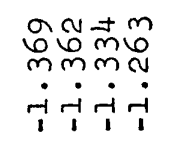 & 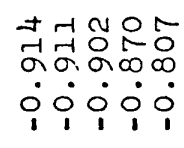 & 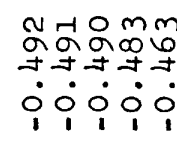 \\
\hline 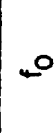 & 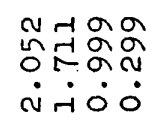 & 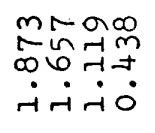 & 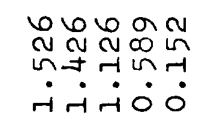 & 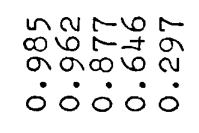 & 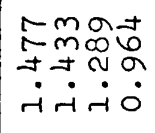 & 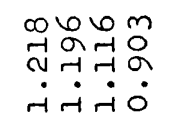 & 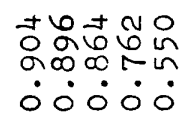 & 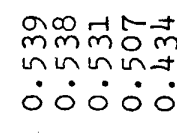 \\
\hline$\Sigma^{\infty}$ & 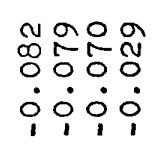 & 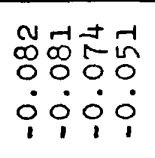 & 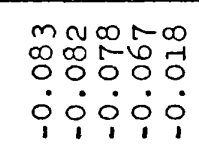 & 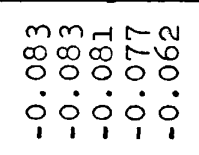 & 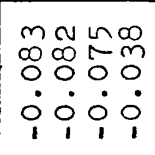 & 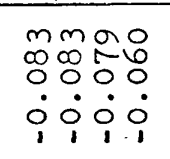 & 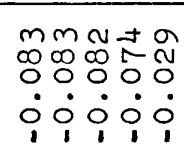 & 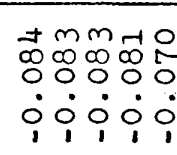 \\
\hline$\Sigma^{*}$ & 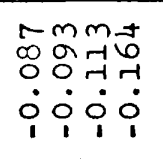 & 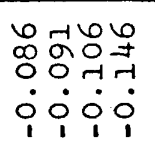 & 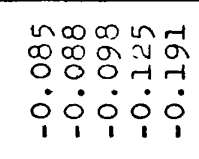 & 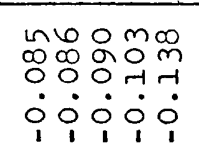 & 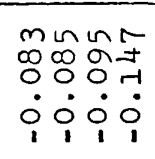 & 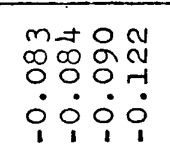 & 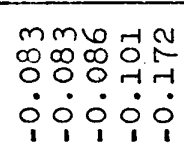 & 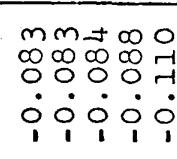 \\
\hline$\Sigma^{m}$ & 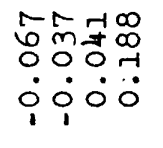 & 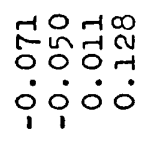 & 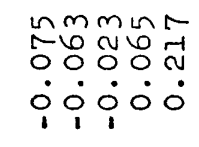 & 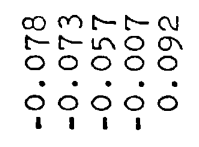 & 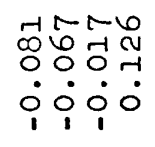 & 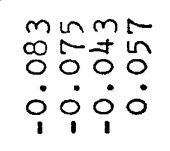 & 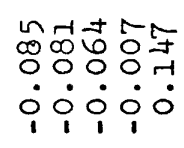 & 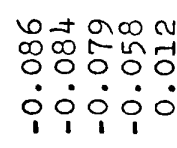 \\
\hline$\Sigma^{N}$ & $\begin{array}{l}\text { mato } \\
\text { ñ } \\
\text { ñ } \\
\dot{0} 0 \dot{0} \\
\dot{0} \dot{0}\end{array}$ & 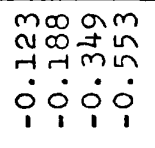 & 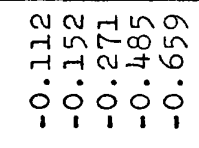 & 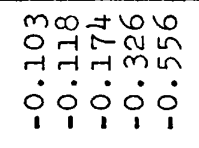 & 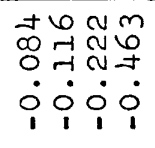 & 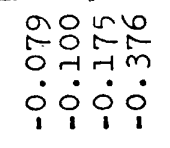 & 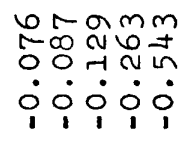 & 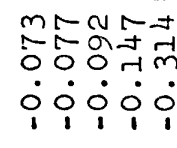 \\
\hline $1 \%$ & $\begin{array}{l}\text { no } 00 \\
\dot{0} \dot{-1} \dot{\sim} \dot{=}\end{array}$ & $\begin{array}{l}\text { no } 00 \\
\dot{0} \dot{-1} \dot{\alpha}\end{array}$ & $\begin{array}{l}n 0000 \\
\dot{0} \dot{-1} \dot{\sim} \dot{A} \dot{\infty}\end{array}$ & 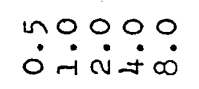 & $\begin{array}{l}\text { no } 00 \\
\dot{0} \dot{\sim} \dot{\sim} \dot{f}\end{array}$ & $\begin{array}{l}n \circ 0 \\
\dot{0} \dot{-1} \dot{\sim} \dot{f}\end{array}$ & 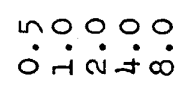 & 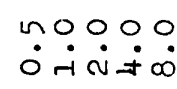 \\
\hline 10 & $\tilde{\sim}$ & $\dot{q}$ & $\ddot{r}$ & - & $\dot{\sim}$ & $\dot{0}$ & n & - \\
\hline & \multicolumn{4}{|c|}{-12} & \multicolumn{4}{|c|}{$-1 N$} \\
\hline ع آع & \\
\hline
\end{tabular}




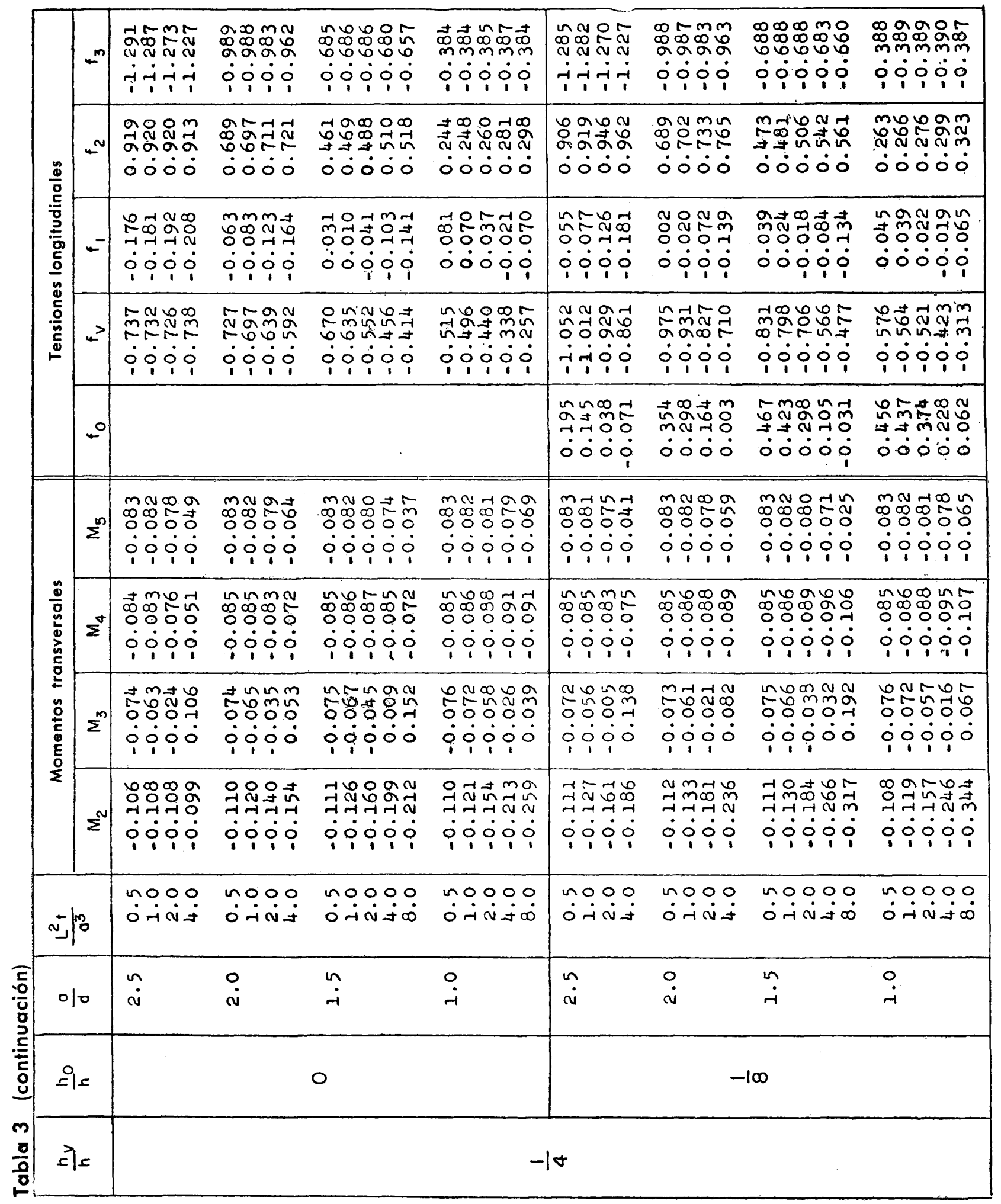




\begin{tabular}{|c|c|c|c|c|c|c|c|c|c|}
\hline & $m$ & 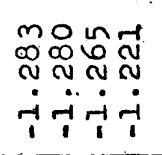 & 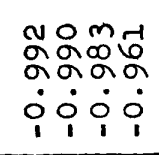 & 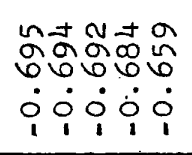 & 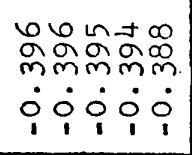 & 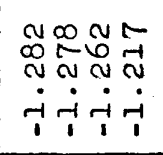 & 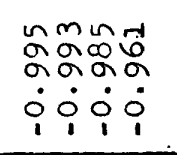 & 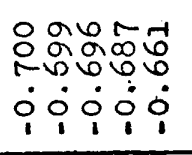 & 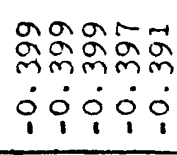 \\
\hline & $\approx$ & 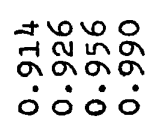 & 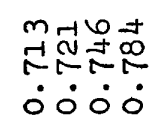 & 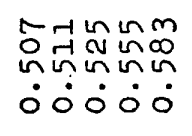 & 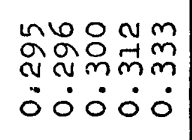 & 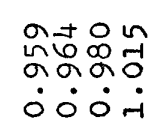 & 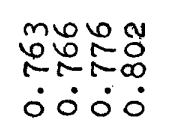 & 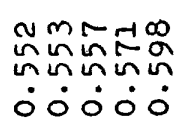 & 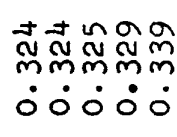 \\
\hline & $=$ & 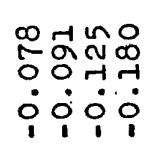 & 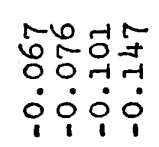 & 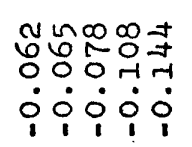 & 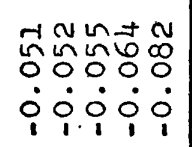 & 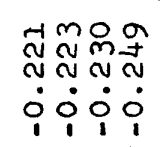 & 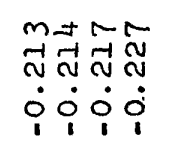 & 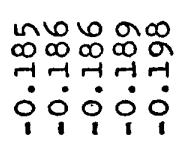 & 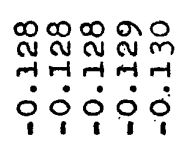 \\
\hline & 2 & 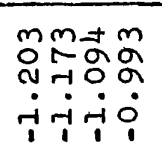 & 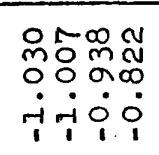 & 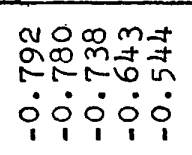 & 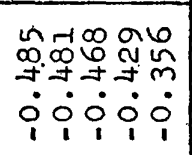 & 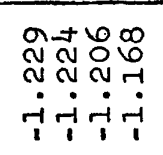 & 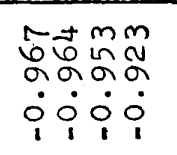 & 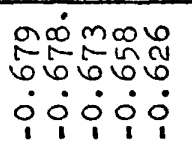 & 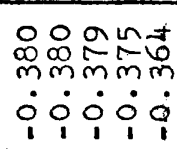 \\
\hline & $\circ$ & 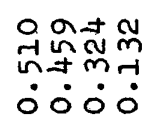 & 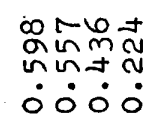 & 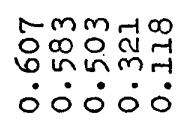 & 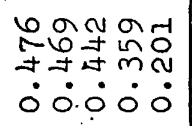 & 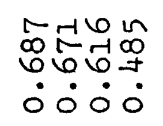 & 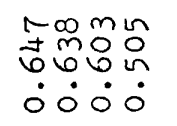 & 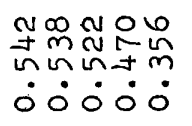 & 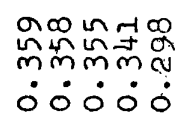 \\
\hline & $\sum^{\infty}$ & 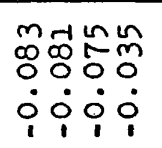 & 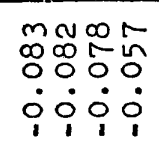 & 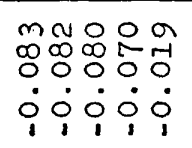 & 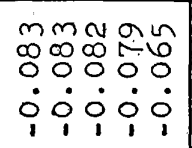 & 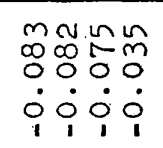 & 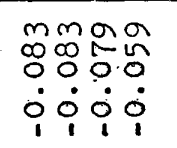 & 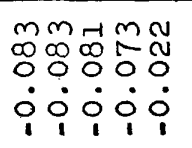 & 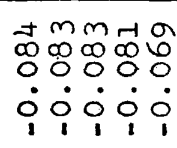 \\
\hline & $\Sigma$ & 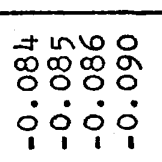 & 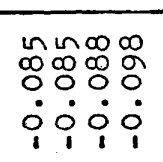 & 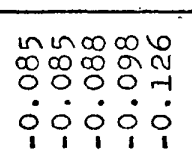 & 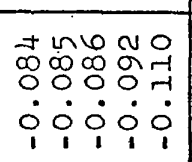 & 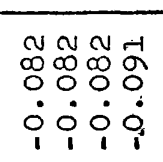 & 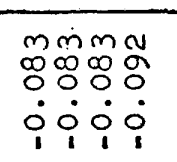 & 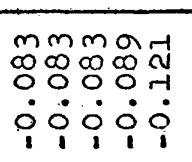 & 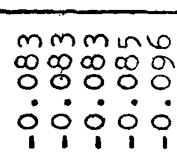 \\
\hline & $\sum^{m}$ & 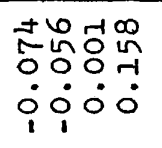 & 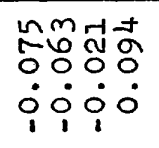 & 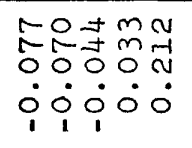 & 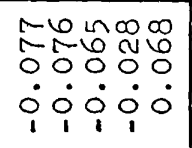 & 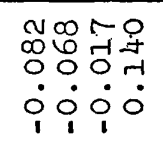 & 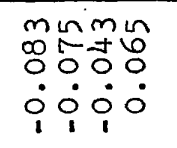 & 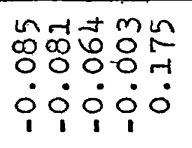 & 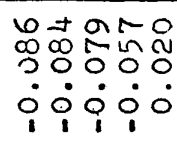 \\
\hline & $\sum^{N}$ & 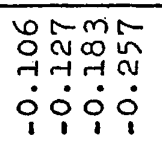 & 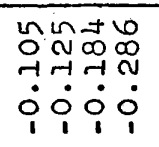 & 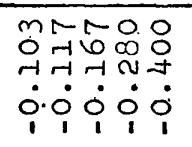 & 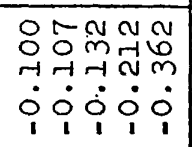 & 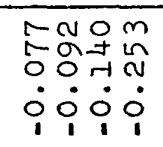 & 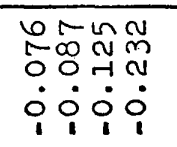 & 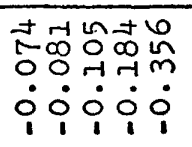 & 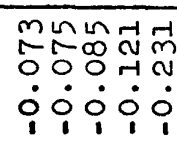 \\
\hline & mo & 〜००: & $\begin{array}{l}\because 00 \% \\
\dot{\circ} \dot{\sim} \dot{j}\end{array}$ & 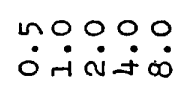 & 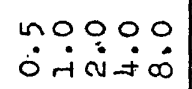 & 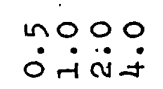 & $\begin{array}{l}\text { no: ०० } \\
\ddot{\circ} \ddot{\sim} \dot{\alpha}=\end{array}$ & 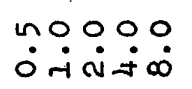 & 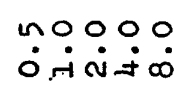 \\
\hline & 10 & $\tilde{n}$ & $\dot{0}$ & $\stackrel{n}{-i}$ & $\stackrel{0}{-1}$ & $\tilde{i}$ & $\dot{\sim}$ & $\ddot{n}$ & $\stackrel{0}{-\dot{-}}$ \\
\hline & ع & \multicolumn{4}{|c|}{-10} & \multicolumn{4}{|c|}{$-k v$} \\
\hline & عا⿰亻 & \multicolumn{8}{|c|}{-10} \\
\hline
\end{tabular}




\begin{tabular}{|c|c|c|c|c|c|c|c|c|c|}
\hline & $=$ & 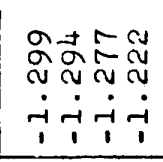 & $\begin{array}{l}0 \% 0 \\
2000 \\
000 \\
000 \\
100 \\
11\end{array}$ & 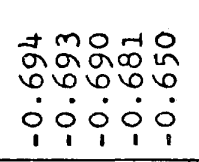 & 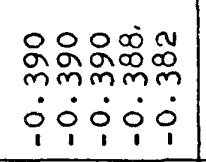 & 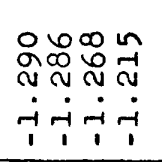 & 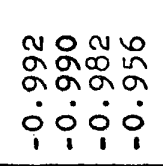 & 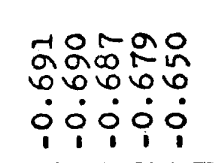 & 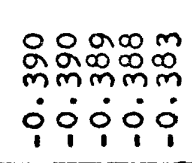 \\
\hline & 4 & 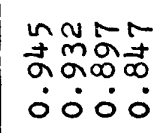 & 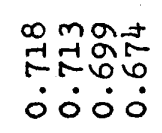 & 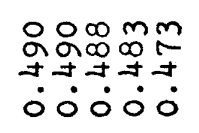 & 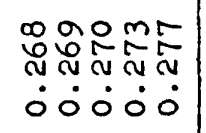 & 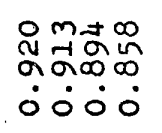 & 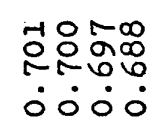 & 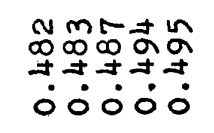 & 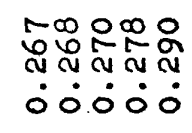 \\
\hline & :- & 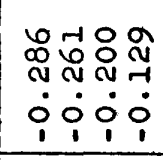 & 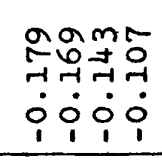 & 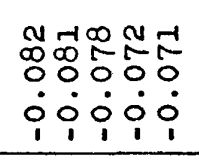 & 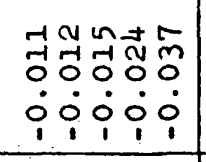 & 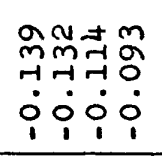 & $\begin{array}{l}\text { 운유뉴 } \\
0000 \\
0000 \\
019\end{array}$ & 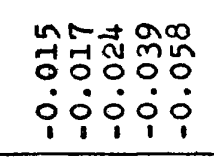 & 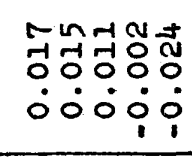 \\
\hline & $=$ & 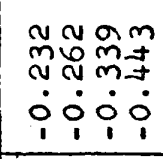 & 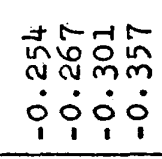 & 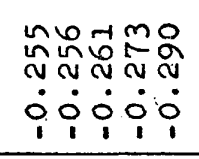 & 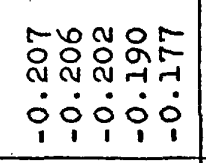 & 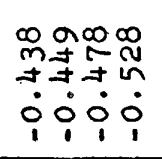 & 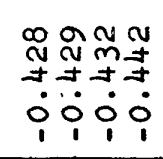 & 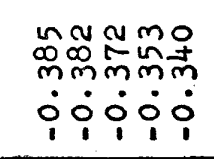 & 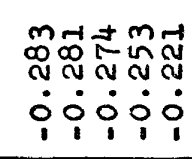 \\
\hline & $\mathcal{C}$ & & & & & 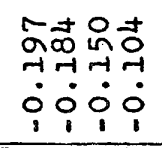 & 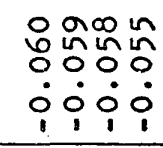 & 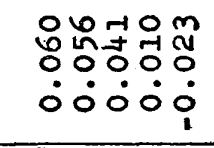 & 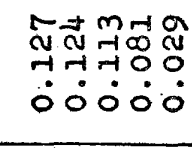 \\
\hline & $\Sigma^{n}$ & 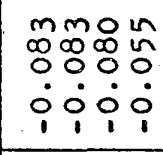 & 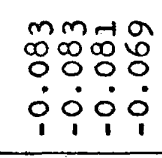 & 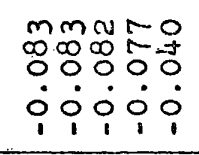 & 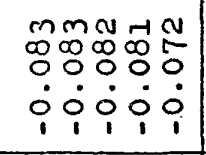 & 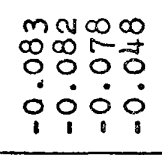 & 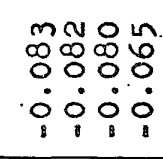 & 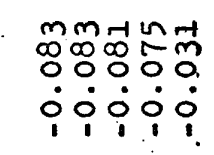 & $\begin{array}{l}m m \infty 00 \\
0000 \\
0000 \\
0000 \\
0\end{array}$ \\
\hline & 这 & 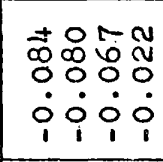 & 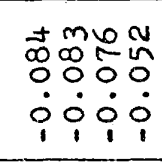 & 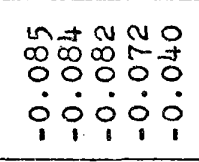 & 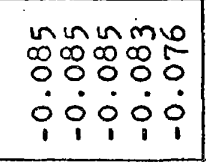 & 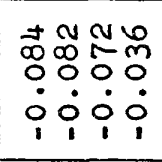 & 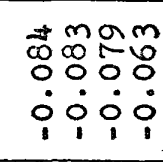 & 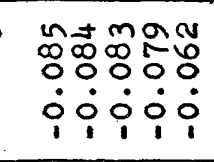 & 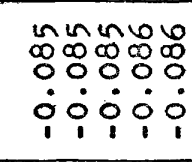 \\
\hline & 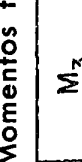 & 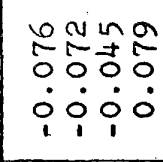 & 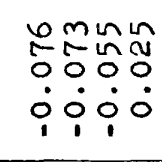 & 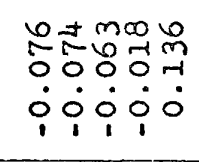 & 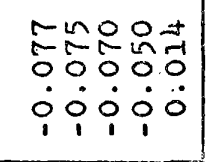 & 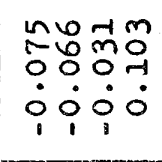 & $\begin{array}{l}0975 \\
0000 \\
000 \\
0 \\
1\end{array}$ & 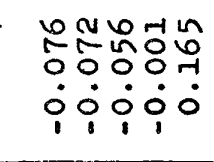 & 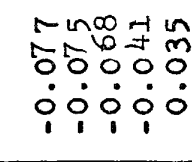 \\
\hline & $\sum$ & 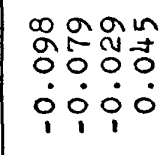 & 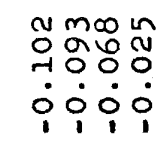 & 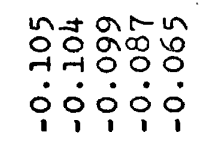 & 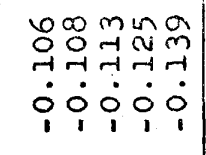 & 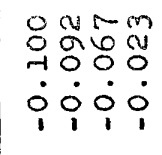 & 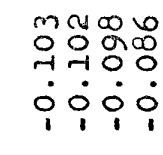 & 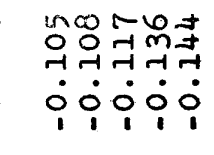 & 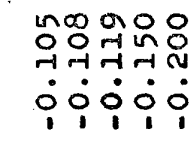 \\
\hline \multirow{3}{*}{ 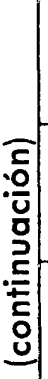 } & o & 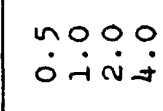 & 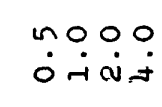 & 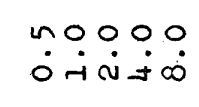 & 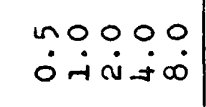 & 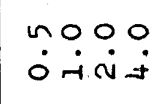 & थ०००० & no०००o & $\begin{array}{l}n 0000 \\
\dot{0} \dot{-1} \dot{0} \dot{0} \dot{0}\end{array}$ \\
\hline & $0 \mid 0$ & $\ddot{i}$ & $\stackrel{\circ}{\dot{\alpha}}$ & $\ddot{n}$ & $\stackrel{0}{-i}$ & $\tilde{i}$ & $\stackrel{0}{\dot{v}}$ & $\ddot{n}$ & $\stackrel{0}{i}$ \\
\hline & ع & \multicolumn{4}{|c|}{0} & \multicolumn{4}{|c|}{-100} \\
\hline & عأl & \multicolumn{8}{|c|}{$m / \infty$} \\
\hline
\end{tabular}




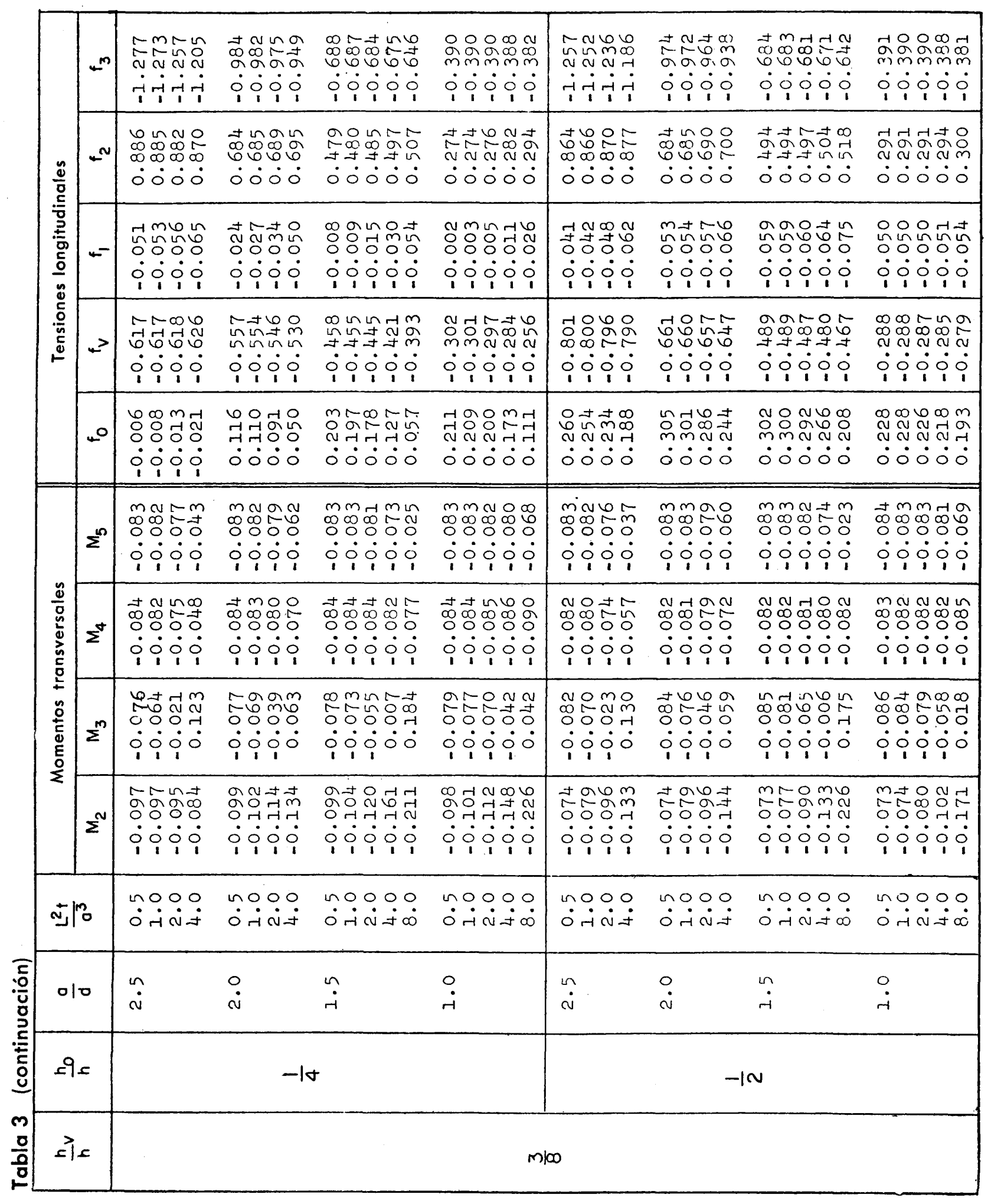




\section{Apéndice}

Aunque el comportamiento de una placa plegada es tridimensional, sin embargo, el análisis y estudio de una estructura como ésta puede reducirse simplemente a la solución de una serie de ecuaciones simultáneas, solución fácilmente realizable por un procedimiento rutinario y aplicando conceptos muy familiares al proyectista. No es preciso ningún tipo de conocimientos distintos de los que dan una familiaridad con la estática, la teoría convencional de flexión y los principios del área de momentos. Las complicaciones que se presentan se deben, principalmente, a la oscura y múltiple interacción de las fuerzas. Debido a esto, es precisa una mayor sistematización de los desarrollos analíticos para las placas plegadas que para estructuras de tipo convencional.

En este orden de ideas, el estudio de las placas plegadas debe empezar considerando las dos acciones, la longitudinal y la transversal, por separado. La primera acción estudia la flexión de las placas individuales considerando el conjunto como una losa continua apoyada sobre las intersecciones. La segunda se refiere, principalmente, a la deflexión de las placas en el plano paralelo a ellas mismas, acción que vamos a llamar «acción placa».

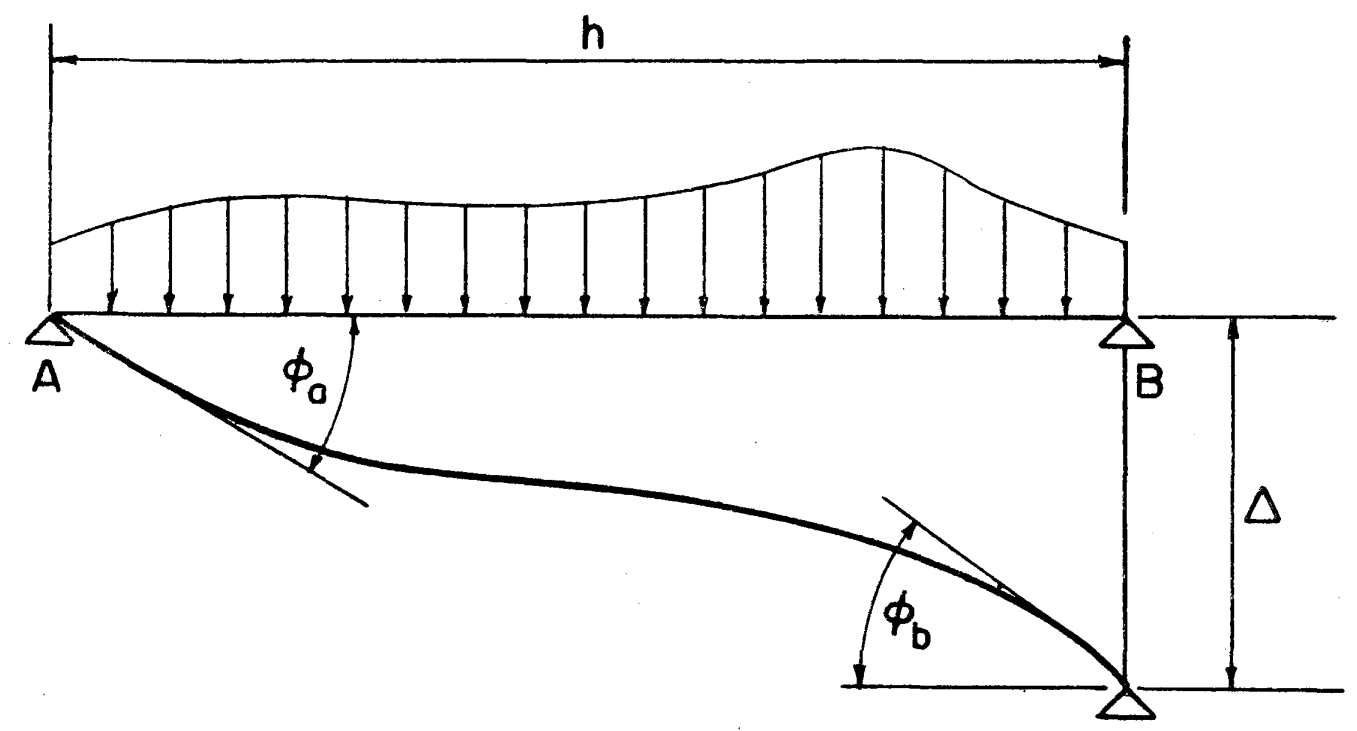

En todo lo anterior, y puesto que la longitud de cada placa en la dirección longitudinal es, generalmente, varias veces la distancia entre los pliegues de las placas, la resistencia de la losa en dirección longitudinal es pequeña y puede ser considerada como una placa unidireccional. Así, pues, la relación entre el momento transversal en las intersecciones es idéntica a la relación análoga en las vigas continuas. Por lo tanto, si a la viga $\mathrm{AB}$, de la figura $\mathrm{Al}$, se la considera como una banda típica limitada por dos pliegues sucesivos, utilizando la notación correspondiente a la distribución de momentos y aplicando el principio primero del área de momentos:

$$
\begin{aligned}
& M_{A B}=K_{A B} \phi_{A B}-C_{B A} K_{B A} \phi_{B A}-\frac{\Delta}{h_{A}}\left(K_{A B}-C_{B A} K_{B A}\right)+M_{A B}^{F} \\
& M_{B A}=C_{A B} K_{A B} \phi_{A B}-K_{B A} \phi_{B A}-\frac{\Delta}{h_{A}}\left(C_{A B} K_{A B}-K_{B A}\right)-M_{B A}^{F}
\end{aligned}
$$

donde:

$\mathrm{M}=$ momento flector. Positivo si produce tracción en la cara inferior de la viga. 
$\mathrm{K}=$ factor de rigidez, que es el momento producido por un giro unitario.

$\mathrm{C}=$ factor de transmisión, negativo.

$\varnothing=$ variación del ángulo respecto a la cuerda. Positivo si gira en el sentido de las agujas del reloj.

$h=$ longitud de placa entre A y B.

$\Delta=$ desplazamiento vertical del extremo B respecto al A. Positivo hacia abajo.

Para vigas prismáticas de anchura unidad es:

$\mathrm{K}=4 \mathrm{E} t^{3} / 12 h=\mathrm{E} t^{3} / 3 h$ y $\mathrm{C}=0,5$, siendo:

$\mathrm{E}=$ módulo de elasticidad.

$t=$ espesor de la placa.

Sustituyendo estos valores en las ecuaciones [la] y [lb], tenemos:

$$
\begin{aligned}
& M_{A B}=\frac{E t^{3}}{3 h_{A}}\left(\phi_{A B}+0.5 \phi_{B A}-1.5 \frac{\Delta}{h_{A}}\right)+M_{A B}^{F} \\
& M_{B A}=\frac{E r^{3}}{3 h_{A}}\left(-0.5 \phi_{A B}-\phi_{B A}+1.5 \frac{\Delta A}{h_{A}}\right)+M_{B A}^{F}
\end{aligned}
$$

Eliminando $\varnothing_{B A}$ de las ecuaciones [2a] : [2b] y sacando factor común, obtendremos:

$$
\phi_{A B}=\frac{4 h_{A}}{E t^{3}}\left(M_{A B}+0.5 M_{B A}-M_{A B}^{F}-0.5 M_{A B}^{F}\right)+\frac{\Delta}{h_{A}}
$$

Al mismo tiempo

$$
\phi_{B A}=-\frac{4 h_{A}^{\prime}}{E P^{3}}\left(M_{B A}+0.5 M_{A B}-M_{B A}^{F}-0.5 M_{A B}^{F}\right)+\frac{\Delta}{h_{A}}
$$

Las ecuaciones anteriores expresan las relaciones básicas necesarias para el estudio y determinación de la variación de magnitud del momento transversal en cada pliegue. La otra ecuación fundamental necesaria es, naturalmente, la que se refiere a la acción longitudinal. Afortunadamente, en este caso el problema se refiere, esencialmente, a la acción, y más específicamente, a la deflexión, de las placas individuales actuando como vigas que van de un soporte a otro. En general, para vigas prismáticas se acostumbra a expresar esta deflexión de una viga en función de la carga dividida por el producto del momento de inercia por el módulo de elasticidad. Sin embargo, en este caso, es más conveniente expresar esta deflexión en función de las tensiones en la fibra extrema. Para hacer esto es necesario conocer la distribución longitudinal de tensiones. Para una viga prismática simplemente apoyada, esta distribución es parabólica. Puesto que la carga muerta y la carga viva son uniformes, en general, en el caso de placas plegadas, parecería deseable, en principio, el suponer una distribución parabólica. Una hipótesis tal nos lleva, sin embargo, a una pequeña incongruencia e introduce una serie de dificultades en el ajuste de las deformaciones longitudinales de dos placas adyacentes. Fácilmente podemos evitar esto suponiendo que la carga y, por lo tanto, las tensiones varían sinusoidalmente en dirección longitudinal.

En este orden de ideas, si se llama $f_{\mathrm{A}}$ y $f_{\mathrm{B}}$ a las tensiones en las dos fibras extremas, superior e inferior de la sección central de una placa, y se supone una relación lineal entre tensio- 
nes y deformaciones como aparece en la figura $\mathrm{A} 2$, el giro de un elemento de longitud $d x$ será:

$$
d \theta=-\left(\frac{f_{A}-f_{B}}{E h_{A}}\right) \operatorname{sen} \frac{\pi x}{L} d x
$$

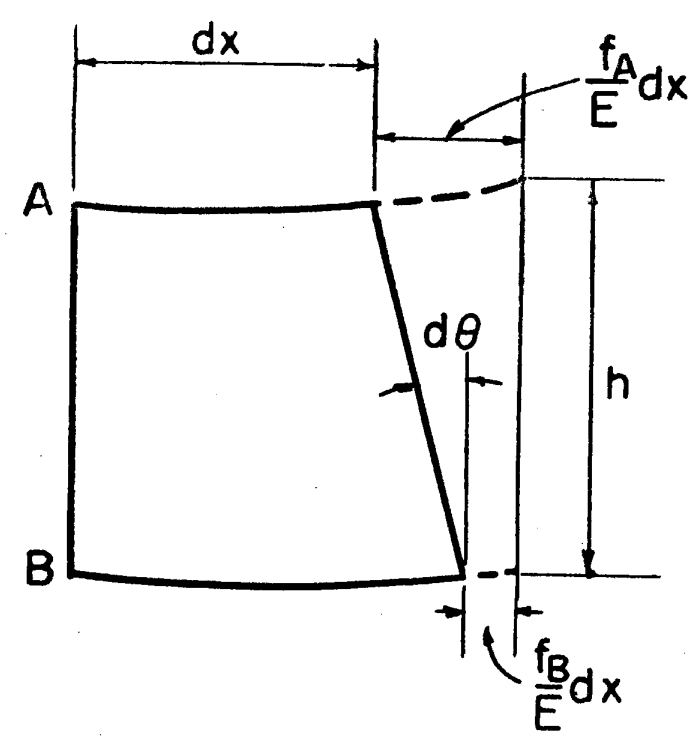

A2

en donde $f_{\mathrm{A}}$ y $f_{\mathrm{B}}$ son positivas si son đe compresión. Al integrar la ecuación [4a] y anular el ángulo total en el centro del vano, el giro aparece determinado por la ecuación:

$$
\theta=\left(\frac{f_{A}-f_{B}}{E h_{A}}\right) \frac{L}{\pi} \cos \frac{\pi x}{L}
$$

Puesto que la derivada de la flecha respecto a $x$ es igual a la variación del ángulo, integrando la ecuación [4b] y anulando la flecha en $X=0$ y $X=L$ :

$$
\delta_{A}=\left(\frac{f_{A}-f_{B}}{E h_{\Delta}}\right)\left(\frac{L}{\pi}\right)^{2}
$$

donde $\delta_{A}$ es la flecha en la sección central de la viga y se la considera positiva dirigida hacia abajo o en dirección de $\mathrm{A}$ a $\mathrm{B}$.

Las tres ecuaciones [3a], $[3 \mathrm{~b}]$ y [4c] son las únicas, de las necesarias para el estudio de las placas plegadas, que dependen de la relación entre tensiones y deformaciones. Los cálculos posteriores se refieren, sobre todo, al equilibrio de fuerzas y al cumplimiento de ciertas relaciones trigonométricas. En la última categoría, puede reducirse el problema a la consideración del efecto de la flexión de una placa sobre las adyacentes. En esta parte del problema, presenta ciertas ventajas al emplear una técnica similar a la utilizada en la distribución de momentos. Así, pues, refiriéndonos a la figura $\mathrm{A} 3$, si la placa $\mathrm{BC}$ está fija en dirección paralela a su plano, pero es capaz de girar alrededor de $\mathrm{C}$ o de flectar, solamente es posible un desplazamiento de la placa $\mathrm{AB}$ desde $\mathrm{B}$ a $\mathrm{B}^{\prime}$ si se produce al mismo tiempo un movimiento en dirección perpendicular a la placa $\mathrm{BC}$. Basándonos en propiedades geométricas del triángulo $\mathrm{BB}^{\prime} \mathrm{B}^{\prime \prime}$ :

$$
\Delta_{B C}^{B}=8 / \operatorname{sen} a_{B}
$$




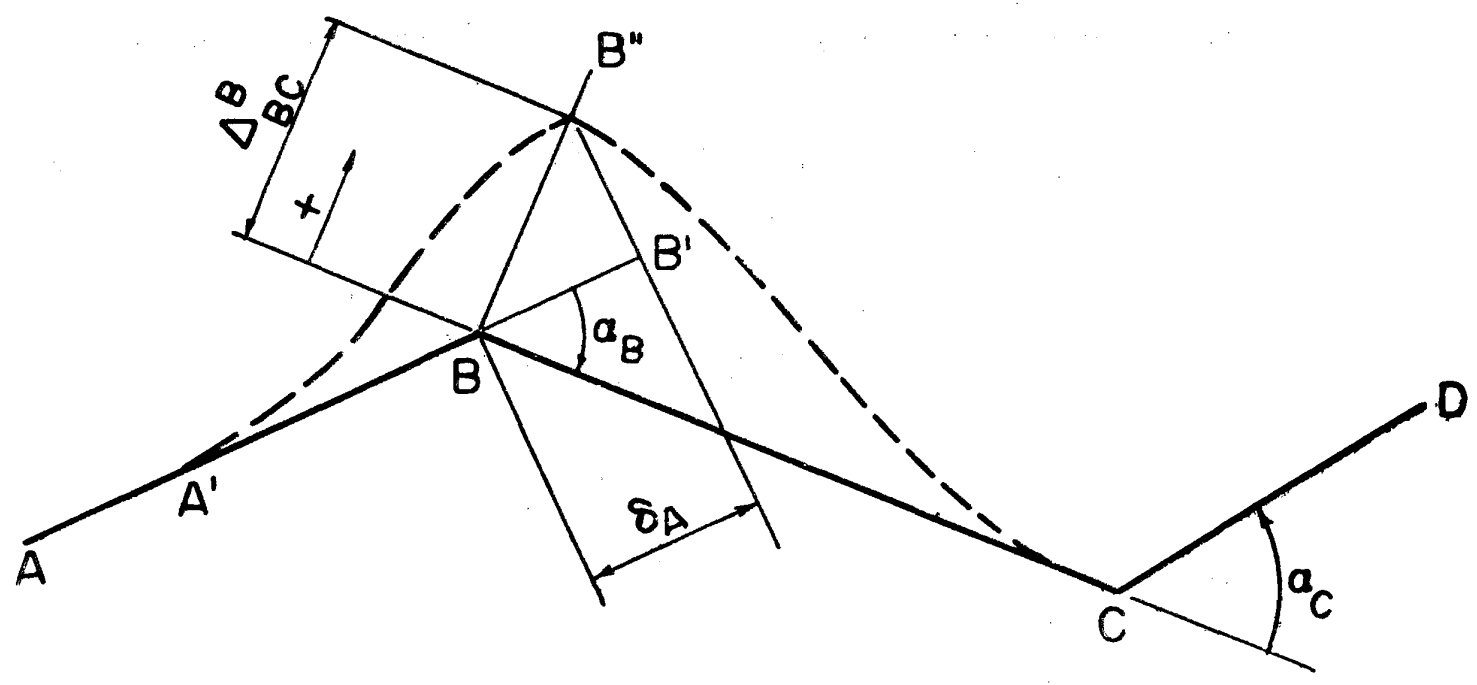

(a) Placa BC fija

donde:

$\alpha_{\mathrm{B}}=$ ángulo formado por la prolongación de la placa $\mathrm{AB}$ con la placa $\mathrm{BC}$. Positivo cuando sigue en su giro el sentido de las agujas del reloj.

$\delta_{\mathrm{A}}=$ flecha de la placa AB. Positiva cuando A se mueve en dirección a $B$.

Fijando la placa $A B$, pero desplazando la $B C$ :

$$
\Delta_{B C}^{B}=-8{ }_{B} \operatorname{ctg} a_{B}
$$

El movimiento total del punto $\mathrm{B}$, normalmente a la línea $\mathrm{BC}$, producido por el desplazamiento de las dos placas $A B$ y $B C$, es, por lo tanto:

$$
\Delta_{B C}^{B}=\delta_{A} / \operatorname{sen} a_{B}-\delta_{B} \operatorname{ctg} a_{B}
$$

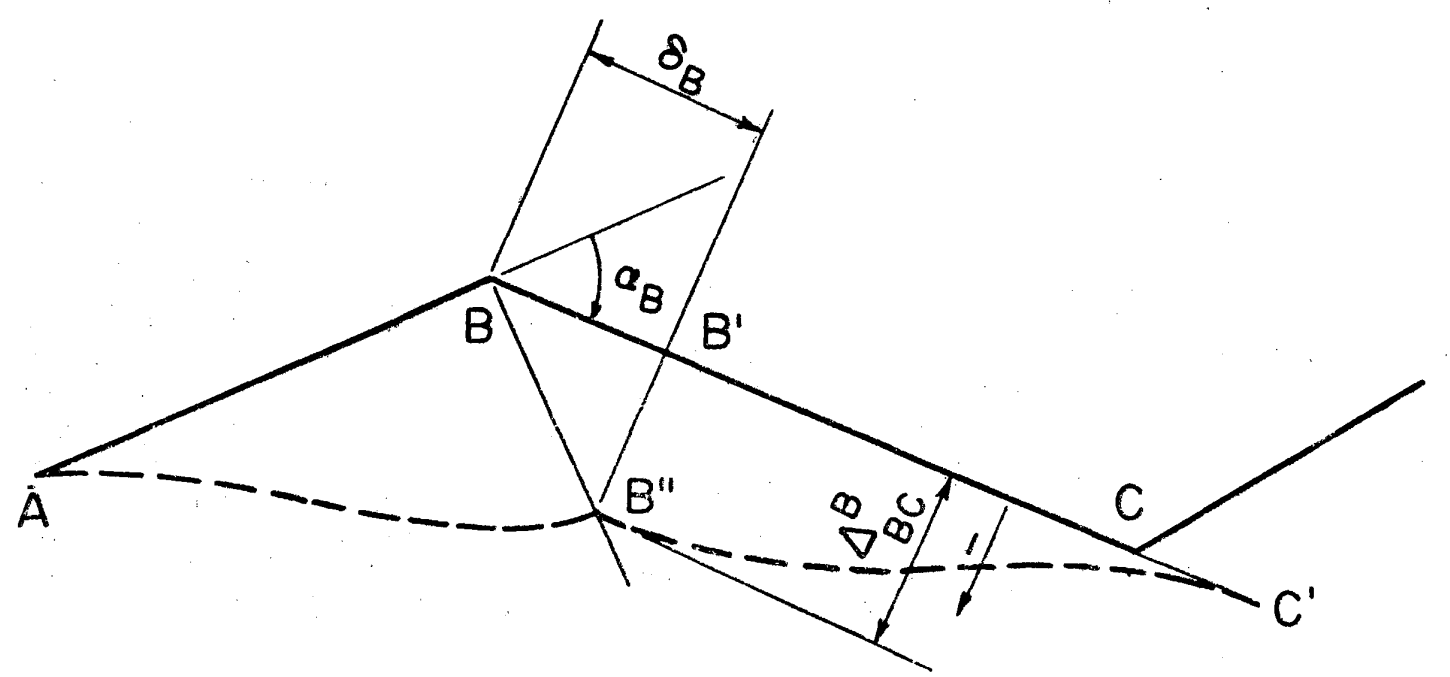

A3

(b) Placa AB fija 


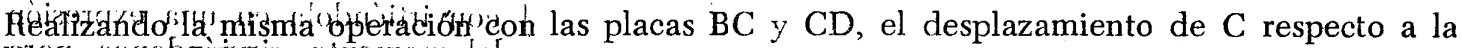

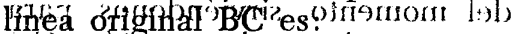

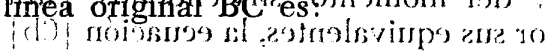

$$
\Delta_{B C}^{C}=-8_{B} c \operatorname{ctg} a_{C}+8_{C} / \operatorname{sen} a_{C}
$$

Puesto que el desplazamiento de $\mathrm{C}$ respecto a $\mathrm{B}$, llamado $\Delta_{\mathrm{BC}}$, es igual a la suma de los valores que se obtienen de $[5 \mathrm{c}]$ y $[5 \mathrm{~d}]$ :

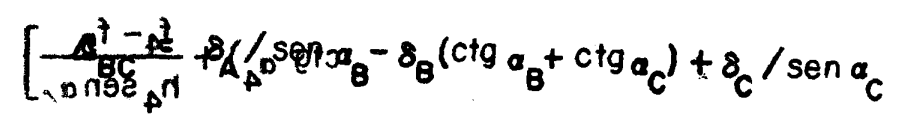

$$
\left\{\left[\frac{a^{7-} a^{7}}{a^{0 n 92} a^{n}}+\left(z^{\infty 1}\right.\right.\right.
$$

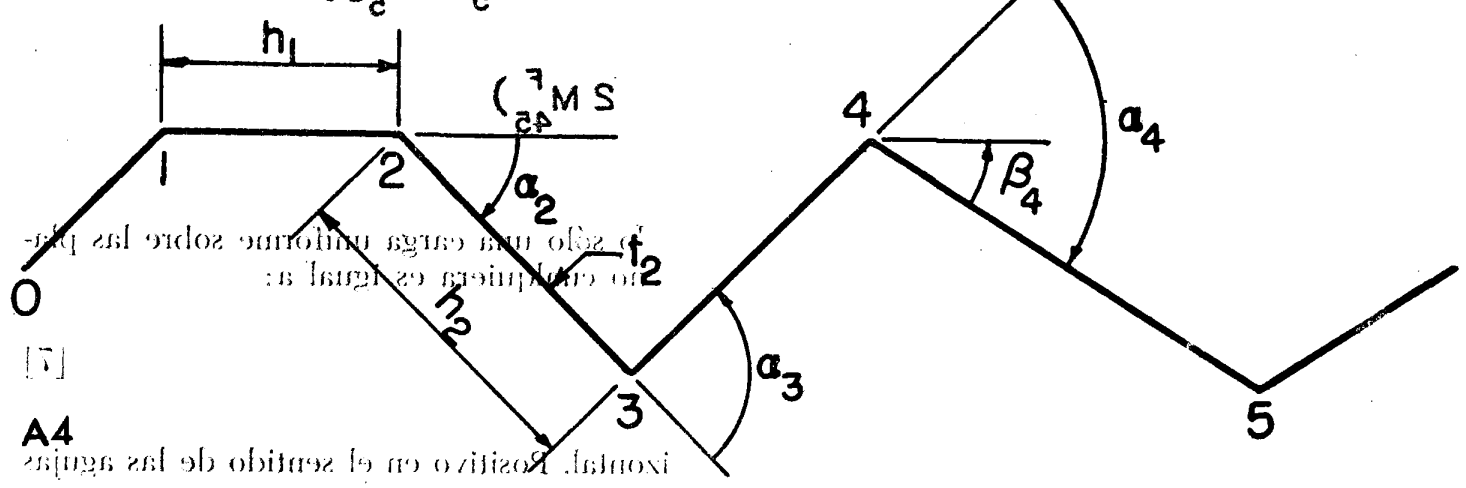

Las ecuaciones generales que acabamos de obtener se aplicarán ahora a una placa plegada como la que aparece en la figura A4. En este caso-por parecer más conveniente-se designan los plieguesı odux númenos en lugarilde!letras. Considerando como punto característico o típico el pliegue 4, el ángulo girado y el momento a la izquierda de 4 deben ser iguales a los corres-

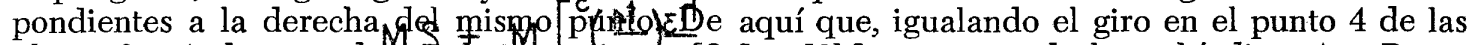
placas 3 y 4, de acuerdo con laspecuaciends $[3 a]$ y [3b], sustituyendo los subíndices $A$ y B por los numerales apropiados, y llamando ă los momentos finales en cada pliegue únicamente con el número del propio pliegue:

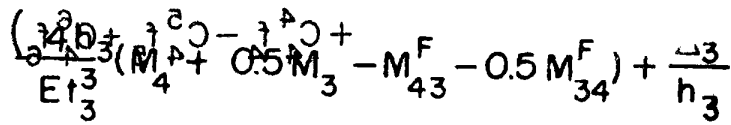

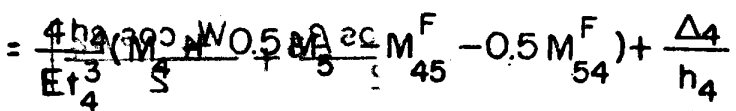

Sustituyendo los incrementos $\Delta$ de la ecuación [6a] por sus equivalentes obtenidos a partir de la [5e] y sacando factor común, obtenemos:

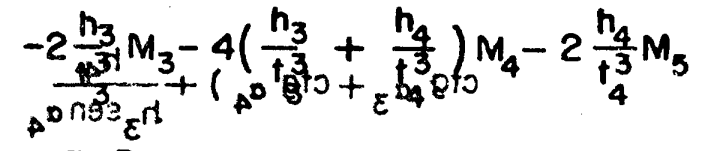

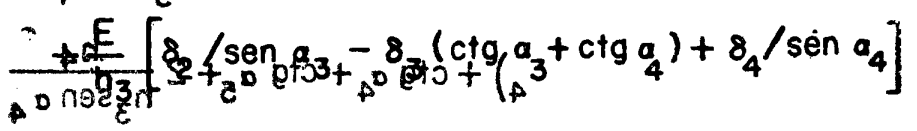

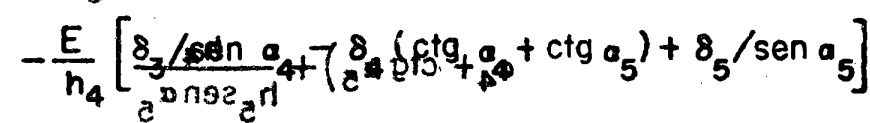

$$
\begin{aligned}
& =-2 \frac{h_{3}}{t_{3}^{3}}\left(M_{34}^{F}+2 M_{43}^{F}\right)-2 \frac{h_{4}}{t_{4}^{3}}\left(M_{54}^{F}+2 M_{45}^{F}\right)
\end{aligned}
$$


Ahora podemos modificar convenientemente la ecuación [6b] convirtiéndola en una expresión que contenga solamente términos en función de la tensión y del momento, sirviéndonos, para ello, de la ecuación [4c]. Si sustituímos el valor de los $\delta$ por sus equivalentes, la ecuación [6b] se reduce a:

$$
\begin{aligned}
& -2 \frac{h_{3}}{t_{3}^{3}} M_{3}-4\left(\frac{h_{3}}{t_{3}^{3}}+\frac{h_{4}}{t_{4}^{3}}\right) M_{4}-2 \frac{n_{4}}{t_{4}^{3}} M_{5} \\
& \left(\frac{L}{\pi}\right)^{2}\left\{+\frac{1}{h_{3}}\left[\frac{f_{2}-f_{3}}{n_{2} \operatorname{sen} a_{3}}-\frac{f_{3}-f_{4}}{h_{3}}\left(\operatorname{ctg} a_{3}+\operatorname{ctg} a_{4}\right)+\frac{f_{4}-f_{5}}{h_{4} \operatorname{sen} a_{4}}\right]\right. \\
& \left.-\frac{1}{h_{4}}\left[\frac{f_{3}-f_{4}}{h_{3} \operatorname{sen} a_{4}}-\frac{f_{4}-f_{5}}{h_{4}}\left(\operatorname{ctg} a_{4}+\operatorname{ctg} a_{5}\right)+\frac{f_{5}-f_{6}}{n_{5} \operatorname{sen} a_{5}}\right]\right\} \\
& =-2 \frac{h_{3}}{f_{3}^{3}}\left(M_{34}^{F}+2 M_{43}^{F}\right)-2 \frac{h_{4}}{t_{4}^{3}}\left(M_{54}^{F}+2 M_{45}^{F}\right)
\end{aligned}
$$

En la ecuación [6c], y puesto que estamos considerando sólo una carga uniforme sobre las placas, el momento de empotramiento perfecto en un vano cualquiera es igual a:

$$
-\frac{W \cos \beta n}{12}
$$

en la cual:

$\beta=$ ángulo que forma la placa con el plano horizontal. Positivo en el sentido de las agujas del reloj.

$\mathrm{W}=$ carga total sobre la placa.

Sustituyendo este valor en la ecuación $[6 \mathrm{c}]$ y sacando factores comunes, obtenemos:

$$
\begin{aligned}
& 2 \frac{h_{3}}{h_{4}}\left(\frac{t_{4}}{t_{3}}\right)^{3} M_{3}+4\left[1+\frac{h_{3}}{h_{4}}\left(\frac{t_{4}}{t_{3}}\right)^{3}\right] M_{4}+2 M_{5} \\
& -\frac{L^{2}}{\pi^{2}}\left(\frac{t_{4}}{h_{4}}\right)^{3}\left(C_{4}^{2} f_{2}-C_{4}^{3} f_{3}+C_{4}^{4} f_{4}-C_{4}^{5} f_{5}+C_{4}^{6} f_{6}\right) \\
& =-n_{4}\left[\left(\frac{h_{3}}{h_{4}}\right)^{2}\left(\frac{t_{4}}{t_{3}}\right)^{3} \frac{W_{3} \cos \beta_{3}}{2}+\frac{W_{4} \cos \beta_{4}}{2}\right]
\end{aligned}
$$

en la cual:

$$
\begin{aligned}
& c_{4}^{2}=\frac{n_{4}^{2}}{h_{2} h_{3} \operatorname{sen} a_{3}} \\
& c_{4}^{3}=\frac{n_{4}^{2}}{h_{2} h_{3} \operatorname{sen} a_{3}}+\left(\frac{h_{4}}{h_{3}}\right)^{2}\left(\operatorname{ctg} a_{3}+\operatorname{ctg} a_{4}\right)+\frac{h_{4}}{h_{3} \operatorname{sen} a_{4}} \\
& c_{4}^{4}=\left(\frac{h_{4}}{h_{3}}\right)^{2}\left(\operatorname{ctg} a_{3}+\operatorname{ctg} a_{4}\right)+\operatorname{ctg} a_{4}+\operatorname{ctg} a_{5}+2 \frac{h_{4}}{h_{3} \operatorname{sen} a_{4}} \\
& c_{4}^{5}=\frac{h_{4}}{h_{3} \operatorname{sen} a_{4}}+\left(\operatorname{ctg} a_{4}+\operatorname{ctg} a_{5}\right)+\frac{h_{4}}{h_{5} \operatorname{sen} a_{5}} \\
& c_{4}^{6}=\frac{h_{4}}{h_{5} \operatorname{sen} a_{5}}
\end{aligned}
$$


La ecuación anterior satisface las exigencias de la continuidad. Podemos obtener otra relación partiendo de la estática y de la teoría convencional de flexión. Si aislamos de una estructura en placa plegada una placa aislada y consideramos únicamente las fuerzas que producen flexión longitudinal, la placa estará sometida, según se indica en la figura A5, a dos fuerzas tangenciales de diferente magnitud y a dos fuerzas normales en cada borde; si, además, se trata de una placa pretensada, también estará sometida al esfuerzo de pretensado. Para hacer compatibles a lo largo de todo el borde las tensiones en los pliegues adyacentes, es necesario que las cargas varíen sinusoidalmente. Sin embargo, por medio de una serie de Fourier, la carga real puede ser, aproximadamente, sustituída por una suma de cargas parciales que varíen así. Desde un punto de vista práctico, sólo es necesario utilizar el primer término de este desarrollo, todo lo más el segundo. Para explicar el proceso de obtención de las ecuaciones, únicamente se utilizará el primer término. En los textos especializados se puede encontrar la forma de ajustar este tipo de desarrollo a la carga de que se trate. Por tanto, basándonos en que la carga normal varía con el seno, y que el esfuerzo cortante lo hace con el coseno, tomando el momento en el centro del vano, la tensión en el punto 4 , en la placa 4 , es:

$$
f_{4}=\frac{1}{f_{4}}\left(\frac{L}{h_{4} \pi}\right)^{2}\left(6 P_{4}-4 \pi \frac{h_{4}}{L} S_{4}-2 \pi \frac{h_{4}}{L} S_{5}\right)+f_{45}^{P}
$$

y en 5:

$$
f_{5}=\frac{1}{t_{4}}\left(\frac{L}{h_{4} \pi}\right)^{2}\left(-6 P_{4}+2 \pi \frac{h_{4}}{L} S_{4}+4 \pi \frac{h_{4}}{L} S_{5}\right)+f_{54}
$$

en donde:

$f=$ tensión creada por el esfuerzo de pretensado considerando cada placa como individual y aislada. El subíndice 45 señala la tensión en el pliegue 4 , y 54 indica la tensión en el pliegue 5 , ambas en la placa 4.

Eliminando $\mathrm{S}_{5}$ de las ecuaciones [9], se obtiene:

$$
2 f_{4}+f_{5}=\frac{1}{f_{4}}\left(\frac{L}{h_{4} \pi}\right)^{2}\left(6 P_{4}-6 \pi \frac{h_{4}}{L} S_{4}\right)+2 f_{45}^{P}+f_{54}^{P}
$$

Repitiendo estas mismas operaciones en la placa 34, tenemos:

$$
\begin{aligned}
& f_{4}=\frac{1}{f_{3}}\left(\frac{L}{h_{3} \pi}\right)^{2}\left(-6 P_{3}+4 \pi \frac{h_{3}}{L} S_{4}+2 \pi \frac{h_{3}}{L} S_{3}\right)+f_{43}^{P} \\
& f_{3}=\frac{1}{f_{3}}\left(\frac{L}{h_{3} \pi}\right)^{2}\left(6 P_{3}-2 \pi \frac{h_{3}}{L} S_{4}-4 \pi \frac{h_{3}}{L} S_{3}\right)+f_{34}^{P}
\end{aligned}
$$

de donde obtenemos:

$$
2 f_{4}+f_{3}=\frac{1}{f_{3}}\left(\frac{L}{h_{3} \pi}\right)^{2}\left(-6 P_{3}+6 \pi \frac{h_{3}}{L} S_{4}\right)+2 f_{43}^{P}+f_{34}^{P}
$$

Las ecuaciones [10] y [12] pueden resolverse como ecuaciones simultáneas para eliminar $\mathbf{S}_{4}$. Multiplicando la ecuación [10] por $t_{4} h_{4}$ y la [12] por $t_{3} h_{3}$ y sumando los dos resultados, obtenemos al sacar factor común:

$$
\begin{aligned}
& h_{3} t_{3} f_{3}+2\left(n_{3} t_{3}+h_{4} t_{4}\right) f_{4}+h_{4} t_{4} f_{5}-6\left(\frac{L}{\pi}\right)^{2}\left(\frac{P_{4}}{h_{4}}-\frac{P_{3}}{h_{3}}\right) \\
& =\left(2 f_{45}^{P}+f_{54}^{P}\right) h_{4} t_{4}+\left(2 f_{43}^{P}+f_{34}^{P}\right) h_{3} t_{3}
\end{aligned}
$$

Para utilizar la ecuación [13] es necesario expresar $\mathrm{P}_{3}$ y $\mathrm{P}_{4}$ en función de la carga y de los mo- 


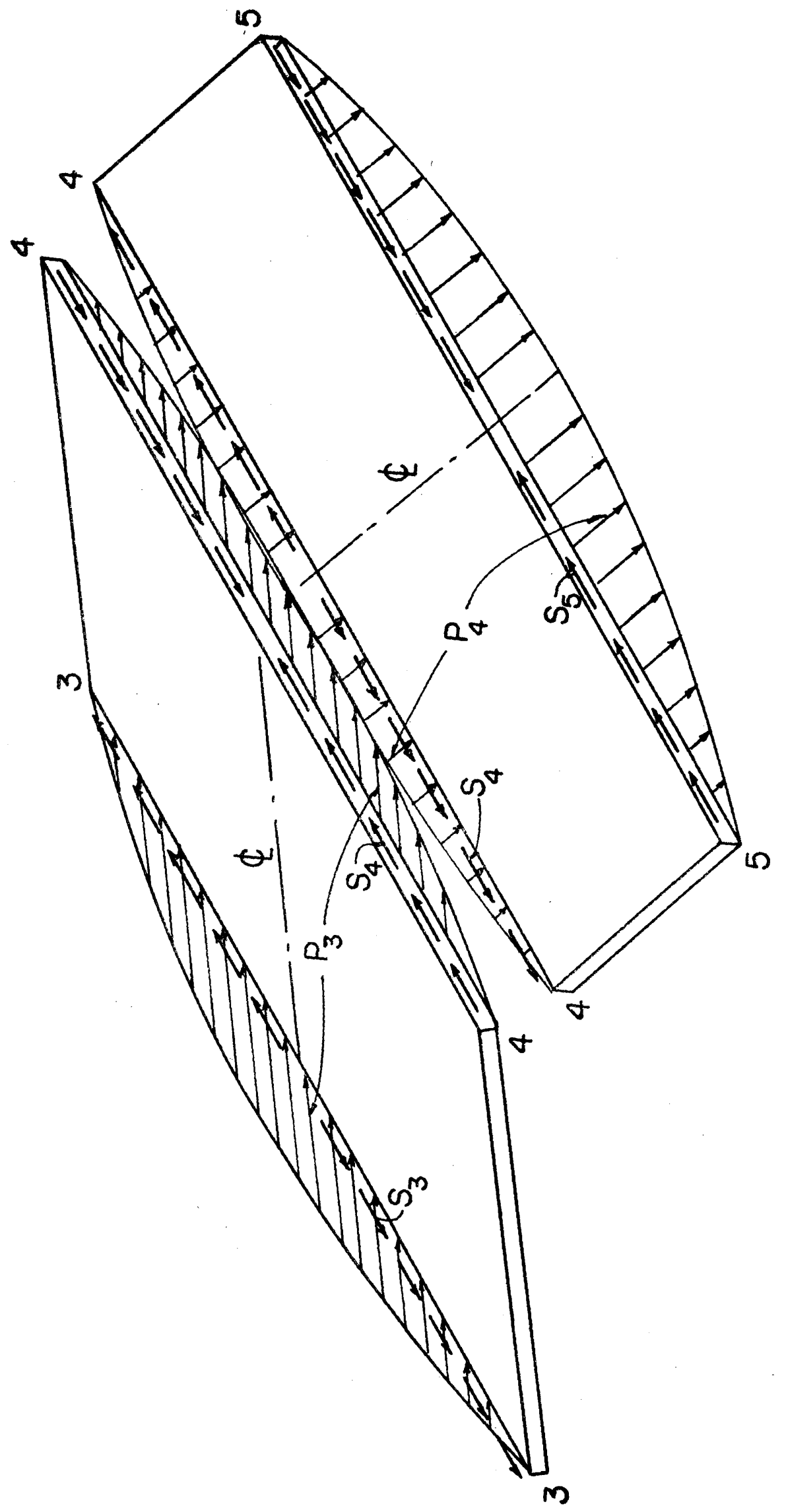


mentos transversales. Si establecemos el equilibrio de fuerzas en la figura A6, considerando cada placa como un elemento aislado, la reacción vertical en el pliegue 4 es:

$$
V_{4}=\frac{M_{3}-M_{4}}{h_{3} \cos \beta_{3}}-\frac{M_{4}-M_{5}}{h_{4} \cos \beta_{4}}+\frac{W_{3}+W_{4}}{2}
$$

y haciendo lo mismo en el pliegue 5, tenemos:

$$
V_{5}=\frac{M_{4}-M_{5}}{h_{4} \cos \beta_{4}}-\frac{M_{5}-M_{6}}{h_{5} \cos \beta_{5}}+\frac{W_{4}+W_{5}}{2}
$$

Puesto que no hay apoyos verticales en los pliegues, estas reacciones deben ser producidas por fuerzas que actúan paralelamente a las placas. Descomponiendo esta reacción en una fuerza paralela a la placa, tenemos:

$$
\begin{aligned}
P_{4} & =V_{4} \frac{\operatorname{sen}\left(90^{\circ}-\beta_{3}\right)}{\operatorname{sen} a_{4}}-V_{5} \frac{\operatorname{sen}\left(90^{\circ}-\beta_{5}\right)}{\operatorname{sen} a_{5}} \\
& =V_{4} \frac{\cos \beta_{3}}{\operatorname{sen} a_{4}}-V_{5} \frac{\cos \beta_{5}}{\operatorname{sen} a_{5}}
\end{aligned}
$$

Sustituyendo los valores de $\mathrm{V}_{4} \mathrm{yV}_{5}$, dados por las ecuaciones [14a] y [14b], en la ecuación $[14 \mathrm{c}]:$

$$
\begin{aligned}
P_{4}= & \frac{M_{3}-M_{4}}{n_{3} \operatorname{sen} a_{4}}-\frac{M_{4}-M_{5}}{h_{4} \cos \beta_{4}}\left(\frac{\cos \beta_{3}}{\operatorname{sen} a_{4}}+\frac{\cos \beta_{5}}{\operatorname{sen} a_{5}}\right)+\frac{M_{5}-M_{6}}{n_{5} \operatorname{sen} a_{5}} \\
& \left(\frac{W_{3}+W_{4}}{2}\right) \frac{\cos \beta_{3}}{\operatorname{sen} a_{4}}-\left(\frac{W_{4}+W_{5}}{2}\right) \frac{\cos \beta_{5}}{\operatorname{sen} a_{5}}
\end{aligned}
$$

Pero de la figura A6:

$$
\beta_{3}=-a_{4}+\beta_{4} \text { y } \quad \beta_{5}=a_{5}+\beta_{4}
$$

Sustituyendo estos valores en el segundo término de la ecuación [14d], obtenemos:

$$
\begin{aligned}
\frac{\cos \left(a_{4}+\beta_{4}\right)}{\cos \beta_{4} \operatorname{sen} a_{4}}+\frac{\cos \left(a_{5}-\beta_{4}\right)}{\cos \beta_{4} \operatorname{sen} a_{5}} & =\frac{\cos a_{4}}{\operatorname{sen} a_{4}}+\frac{\operatorname{sen} \beta_{4}}{\cos \beta_{4}}+\frac{\cos a_{5}}{\operatorname{sen} a_{5}}-\frac{\operatorname{sen} \beta_{4}}{\cos \beta_{4}} \\
& =\operatorname{ctg} a_{4}+\operatorname{ctg} a_{5}
\end{aligned}
$$

Por tanto, la ecuación [14d] se reduce a:

$$
\begin{aligned}
& P_{4}=\frac{M_{3}-M_{4}}{h_{3} \operatorname{sen} a_{4}}-\frac{M_{4}-M_{5}}{h_{4}}\left(\operatorname{ctg} a_{4}+\operatorname{ctg} a_{5}\right)+\frac{M_{5}-M_{6}}{h_{5} \operatorname{sen} a_{5}} \\
& +\left(\frac{W_{3}+W_{4}}{2}\right) \frac{\cos \beta_{3}}{\operatorname{sen} a_{4}}-\left(\frac{W_{4}+W_{5}}{2}\right) \frac{\cos \beta_{5}}{\operatorname{sen} a_{5}}
\end{aligned}
$$




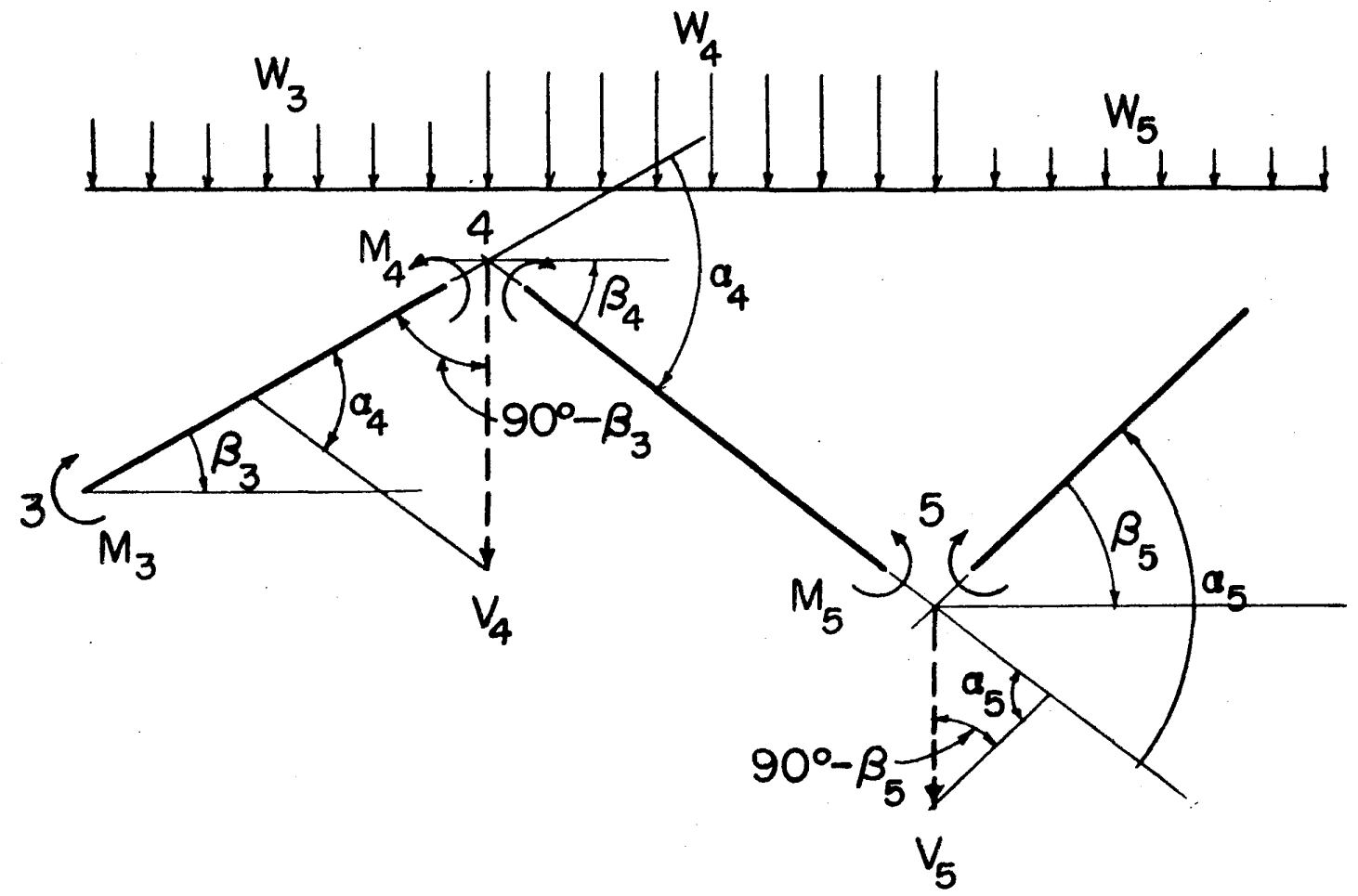

A6

Realizando operaciones similares con las placas 2 y 3 :

$$
\begin{aligned}
& P_{3}=\frac{M_{2}-M_{3}}{h_{2} \operatorname{sen} a_{3}}-\frac{M_{3}-M_{4}}{h_{3}}\left(\operatorname{ctg} a_{3}+\operatorname{ctg} a_{4}\right)+\frac{M_{4}-M_{5}}{h_{4} \operatorname{sen} a_{4}} \\
& +\left(\frac{W_{2}+W_{3}}{2}\right) \frac{\cos \beta_{2}}{\operatorname{sen} a_{3}}-\left(\frac{W_{3}+W_{4}}{2}\right) \frac{\cos \beta_{4}}{\operatorname{sen} a_{4}}
\end{aligned}
$$

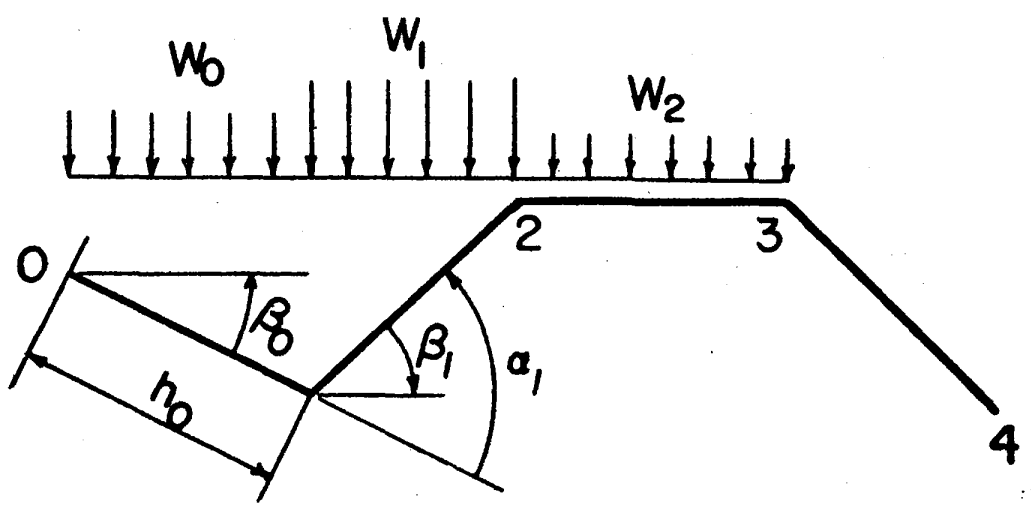


Al sustituir el valor de $\mathrm{P}_{3}$ y el de $\mathrm{P}_{4}$, obtenidos de las ecuaciones [15a] y [15b] en la ecuación [13], y reducir términos semejantes, obtenemos:

$$
\begin{aligned}
& \frac{h_{3}^{t} f_{3}}{h_{4}^{\dagger}} f_{3}+2\left(1+\frac{h_{3} t_{3}}{h_{4}^{t}}\right) f_{4}+f_{5}+\frac{6}{t_{4} h_{4}}\left(\frac{L}{\pi h_{4}}\right)^{2}\left(C_{4}^{2} M_{2}-C_{4}^{3} M_{3}+C_{4}^{4} M_{4}-C_{4}^{5} M_{5}+C_{4}^{6} M_{6}\right) \\
= & -\frac{3}{f_{4}}\left(\frac{L}{\pi h_{4}}\right)^{2}\left[\left(W_{2}+W_{3}\right) \frac{h_{4} \cos \beta_{2}}{h_{3} \operatorname{sen} a_{3}}-\left(W_{3}+W_{4}\right)\left(\frac{\cos \beta_{3}}{\operatorname{sen} a_{4}}+\frac{h_{4} \cos \beta_{4}}{h_{3} \operatorname{sen} a_{4}}\right)+\left(W_{4}+W_{5}\right) \frac{\cos \beta_{5}}{\operatorname{sen} a_{5}}\right] \\
+ & \frac{h_{3} t_{3}}{n_{4}^{\dagger}}\left(2 f_{43}^{P}+f_{34}^{P}\right)+2 f_{45}^{P}+f_{54}^{P}
\end{aligned}
$$

Las ecuaciones [8] y [16] dan dos relaciones distintas que ligan entre sí a las tensiones, momentos y cargas en el pliegue 4. Esta relación, con los subíndices apropiados, es válida para cualquier otro pliegue interior. Por tanto, para cada uno de estos pliegues interiores pueden escribirse dos ecuaciones de naturaleza semejante a estas dos [8] y [16]. Puesto que solamente existen dos incógnitas por pliegue, es evidente que puede establecerse, para obtener los esfuerzos y momentos desconocidos, un sistema de ecuaciones lineales. En relación con esto, han de tenerse en cuenta las ventajas que pueden extraerse de las condiciones de simetría y que permiten reducir el trabajo necesario. Así, si el pliegue 4 está en el eje de simetría, se tiene que, para carga también simétrica: $f_{2}=f_{\mathrm{B}} ; f_{3}=f_{5} ; \mathrm{M}_{2}=\mathrm{M}_{6} ; \mathrm{M}_{3}=\mathrm{M}_{5}$. Al modificar las ecuaciones, teniendo en cuenta estas relaciones, se reduce el número de incógnitas.

Dado que las ecuaciones fundamentales en un pliegue cualquiera ligan entre sí el momento y las tensiones en los pliegues adyacentes y en los siguientes a los adyacentes al propio pliegue, no son aplicables en las extremidades transversales de una estructura en placa plegada. La relación válida para estos extremos es bien conocida. Por ejemplo: en la figura A7 el momento en 0 ha de ser nulo, mientras que el momento en 1, por consideraciones estáticas, debe ser igual al momento de ménsula de la carga a la izquierda de 1. En consecuencia, en cada uno de estos pliegues queda únicamente una incógnita: la tensión longitudinal y, por lo tanto, sólo se necesita una ecuación.

Conocidos los momentos, debe considerarse únicamente el equilibrio estático y la flexión de las placas, como vigas que resisten longitudinalmente. Si en la figura A7 se considera la placa I como un elemento aislado, sin esfuerzo tangencial en $0\left(S_{0}=0\right)$, de acuerdo con la ecuación [10].

$$
2 f_{0}+f_{1}=\frac{6}{f_{0}}\left(\frac{L}{h_{0}^{\pi}}\right)^{2} P_{0}+2 f_{01}^{P}+f_{10}^{p}
$$

Puesto que no hay ningún elemento a la izquierda del 0 , en la ecuación [14c]:

$$
P_{0}=-v_{1} \frac{\cos \beta_{1}}{\operatorname{sen} a_{1}}
$$

Puesto que en la ecuación [14a]:

$$
v_{1}=w_{0}+\frac{w_{1}}{2}-\frac{M_{1}-M_{2}}{h_{1} \cos \beta_{1}}
$$

sustituyendo $V_{1}$ en la ecuación [18] y haciendo

$$
M_{1}=-\frac{W_{0} h_{0} \cos \beta_{0}}{2}
$$


obtenemos:

$$
P_{0}=-\left[\left(W_{0}+\frac{W_{1}}{2}\right)+\frac{W_{0} h_{0} \cos \beta_{0}}{2 h_{1} \cos \beta_{1}}\right] \frac{\cos \beta_{1}}{\operatorname{sen} a_{1}}-\frac{M_{2}}{h_{1} \operatorname{sen} a_{1}}
$$

Sustituyendo este valor en la ecuación [17], se tiene:

$$
\begin{aligned}
& 2 f_{0}+f_{1}+\frac{6}{f_{0} h_{0}}\left(\frac{L}{h_{0} \pi}\right)^{2} \frac{h_{0}}{h_{1} \operatorname{sen} a_{1}} M_{2} \\
& =-\frac{3}{t_{0}}\left(\frac{L}{h_{0} \pi}\right)^{2}\left(2 W_{0}+W_{1}+W_{0} \frac{h_{0} \cos \beta_{0}}{h_{1} \cos \beta_{1}}\right) \frac{\cos \beta_{1}}{\operatorname{sen} \alpha_{1}}+2 f_{01}^{p}+f_{10}^{p}
\end{aligned}
$$

La obtención de la ecuación válida para el pliegue 1, puede iniciarse volviendo a restablecer la ecuación [13] con los subíndices apropiados. Para el pliegue 1, tenemos:

$$
\begin{aligned}
& h_{0} t_{0} f_{0}+2\left(h_{0} t_{0}+h_{1} t_{1}\right) f_{1}+h_{1} t_{12} f_{2}-6\left(\frac{L}{\pi}\right)^{2}\left(\frac{P_{1}}{h_{1}}-\frac{P_{0}}{h_{0}}\right) \\
& =\left(2 f_{12}^{P}+f_{21}^{P}\right) h_{1} t_{1}+\left(2 f_{10}^{P}+f_{01}^{P}\right) h_{0} t_{0}
\end{aligned}
$$

Pero según las ecuaciones [15a]:

$$
\begin{aligned}
& P_{1}=\frac{M_{0}-M_{1}}{h_{0} \operatorname{sen} a_{1}}-\frac{M_{1}-M_{2}}{h_{1}}\left(\operatorname{ctg} a_{1}+\operatorname{ctg} a_{2}\right)+\frac{M_{2}-M_{3}}{h_{2} \operatorname{sen} a_{2}} \\
& +\left(\frac{W_{0}+W_{1}}{2}\right) \frac{\cos \beta_{0}}{\operatorname{sen} a_{1}}-\left(\frac{W_{1}+W_{2}}{2}\right) \frac{\cos \beta_{2}}{\operatorname{sen} a_{2}}
\end{aligned}
$$

Al sustituir el valor de $\mathrm{P}_{0}$ y $\mathrm{P}_{1}$, dado por las ecuaciones [19b] y [22], respectivamente, la ecuación [21] se reduce a:

$$
\begin{aligned}
& \frac{n_{0} t_{0} f_{0}}{n_{1} t_{1}}+2\left(1+\frac{h_{0} t_{0}}{n_{1} f_{1}}\right) f_{1}+f_{2}-\frac{6}{n_{1} f_{1}}\left(\frac{L}{n_{1} \pi}\right)^{2}\left(c_{1}^{2} M_{2}-c_{1}^{3} M_{3}\right) \\
& =\frac{3}{t_{1}}\left(\frac{L}{\pi n}\right)_{1}^{2}\left[\left(2 W_{0}+W_{1}\right)\left(\frac{\cos \beta_{0}}{\operatorname{sen} a_{1}}+\frac{h_{1} \cos \beta_{1}}{h_{0} \operatorname{sen} a_{1}}\right)-\left(W_{1}+W_{2}\right) \frac{\cos \beta_{2}}{\operatorname{sen} a_{2}}+w_{0} \frac{n_{0}}{n_{1}} \cos \beta_{0} C_{1}\right] \\
& +2 f_{12}^{p}+f_{21}^{p}+\frac{h_{0} t_{0}}{h_{1} t_{1}}\left(2 f_{10}^{p}+f_{01}^{p}\right)
\end{aligned}
$$

en la cual:

$$
\begin{aligned}
& c_{1}=\left(\operatorname{ctg} a_{1}+\operatorname{ctg} a_{2}\right)+\frac{n_{1}}{n_{0} \operatorname{sen} a_{1}} \\
& c_{1}^{2}=\operatorname{ctg} a_{1}+\operatorname{ctg} a_{2}+\frac{n_{1}}{n_{2} \operatorname{sen} a_{2}}+\frac{n_{1}}{n_{0} \operatorname{sen} a_{1}} \\
& c_{1}^{3}=\frac{n_{1}}{n_{2} \operatorname{sen} a_{2}}
\end{aligned}
$$

Cuando la luz longitudinal cle una placa plegada no pretensada es grande, el momento trans- 
versal en el pliegue 2 puede llegar a ser mayor que el que puede resistir una losa de espesor normal. En estos casos, un elemento vertical será, las más de las veces, una considerable ayuda. El análisis de una estructura formada por la intersección de tres placas, aunque algo más complejo, sigue el mismo proceso que se ha desarrollado previamente. Así, si consideramos la placa 0 , en la figura $A 8$, como un elemento aislado, como en la ecuación [10]:

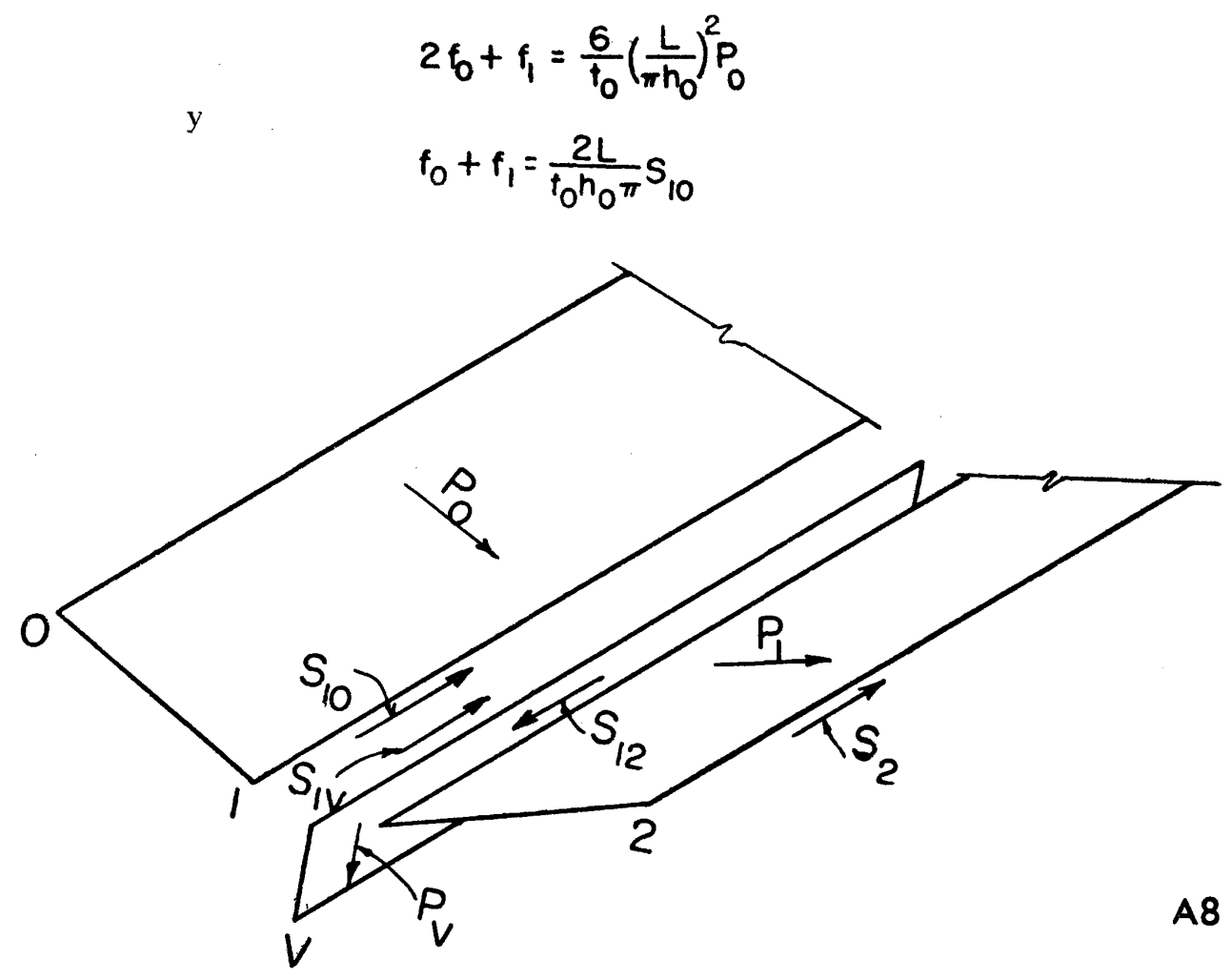

La solución de estas ecuaciones nos da:

$$
\begin{aligned}
& f_{0}=\frac{1}{t_{0}}\left(\frac{L}{\pi h}\right)_{0}^{2}\left(6 P_{0}-\frac{2 \pi h_{0}}{L} S_{10}\right) \\
& f_{1}=\frac{1}{t_{0}}\left(\frac{L}{\pi h}\right)^{2}\left(-6 P_{0}+\frac{4 \pi h_{0}}{L} S_{10}\right)
\end{aligned}
$$

Si en la placa $\mathrm{V}$ se considera que $\mathrm{P}_{\mathrm{V}}$ actúa verticalmente hacia abajo, entonces:

$$
\begin{aligned}
& f_{1}+2 f_{v}=\frac{-6}{f_{v}}\left(\frac{L}{\pi h_{v}}\right)^{2} P_{v} \\
& f_{1}+f_{v}=\frac{2 L}{f_{v} h_{v} \pi} S_{1 v}
\end{aligned}
$$

En la placa 1:

$$
2 f_{1}+f_{2}=\frac{6}{f_{1}}\left(\frac{L}{\pi h_{1}}\right)^{2} P_{1}-\frac{6}{\pi t_{1} h_{1}} S_{12}
$$


Pero con la convención de signos adoptada en la figura A8:

$$
s_{12}=s_{10}+s_{N}
$$

De aquí, si se sustituye la expresión de $\mathrm{S}_{10} \mathrm{y} \mathrm{S}_{10}$, dada por las ecuaciones [24d] y [24f], en la $[24 \mathrm{~g}]$, al reducir términos semejantes se obtiene:

$$
3\left(\frac{h_{0} t_{0}}{h_{1} t_{1}}\right) f_{0}+\left(2+3 \frac{h_{0} t_{0}}{h_{1} t_{1}}+3 \frac{h_{v} t_{v}}{h_{1} t_{1}}\right) f_{1}+f_{2}+3 \frac{h_{v} t_{v}}{h_{1} t_{1}} f_{v}=\frac{6}{t_{1}}\left(\frac{L}{h_{1} \pi}\right)^{2} P_{1}
$$

Como se ha hecho en los cálculos anteriores, es ahora preciso expresar $P_{1}$ en función de la carga y de los momentos en las placas adyacentes. Por medio de la ecuación [4a], añadiéndose la carga sobre la placa $V$, tenemos:

$$
v_{1}=\frac{W_{0}+W_{1}}{2}+\frac{M_{0}-M_{1}}{h_{0} \cos \beta_{0}}-\frac{M_{1}-M_{2}}{h_{1} \cos \beta_{1}}+W_{v}
$$

y

$$
v_{2}=\frac{W_{1}+W_{2}}{2}+\frac{M_{1}-M_{2}}{h_{1} \cos \beta_{1}}-\frac{M_{2}-M_{3}}{h_{2} \cos \beta_{2}}
$$

Puesto que parte de la reacción vertical en el pliegue 1 se resiste por la propia placa $\mathrm{V}$, la relación dada por la ecuación [14c] debe modificarse de acuerdo con:

$$
p_{0}=-\left(v_{1}-p_{v}\right) \frac{\cos \beta}{\operatorname{sen} a_{1}}
$$

y

$$
P_{1}=V_{1} \frac{\cos \beta_{0}}{\operatorname{sen} a_{1}}-V_{2} \frac{\cos \beta_{2}}{\operatorname{sen} \alpha_{2}}-P_{v} \frac{\cos \beta_{0}}{\operatorname{sen} a_{1}}
$$

las cuales, mediante las ecuaciones [24e], [26a] y [26b], pueden ser establecidas de forma análoga a la ecuación [15a], quedando:

$$
\begin{aligned}
P_{1}= & -\frac{M_{1}}{h_{0} \operatorname{sen} a_{1}}-\frac{M_{1}-M_{2}}{h_{1}}\left(\operatorname{ctg} a_{1}+\operatorname{ctg} a_{2}\right)+\frac{M_{2}-M_{3}}{h_{2} \operatorname{sen} a_{2}} \\
& +\left(\frac{W_{0}+W_{1}}{2}\right) \frac{\cos \beta_{0}}{\operatorname{sen} a_{1}}-\frac{W_{1}+W_{2}}{2} \frac{\cos \beta_{2}}{\operatorname{sen} a_{2}}+\left[W_{v}+\frac{t_{1}}{6}\left(\frac{h_{V} \pi}{L}\right)^{2}\left(f_{1}+2 f_{v}\right]\right) \frac{\cos \beta_{0}}{\operatorname{sen} a_{1}}
\end{aligned}
$$

Sustituyendo este valor en la ecuación [25], obtenemos:

$$
\begin{aligned}
& 3 \frac{h_{0} t_{0}}{h_{1} t_{1}} f_{0}+\left[2+3 \frac{h_{0} t_{0}}{h_{1} t_{1}}+3 \frac{h_{v} t_{v}}{h_{1} t_{1}}-\frac{t_{y}}{t_{1}}\left(\frac{h_{v}}{h_{1}}\right)^{2} \frac{\cos \beta_{0}}{\operatorname{sen} a_{1}}\right] \\
& +f_{2}+\left[\frac{3 h_{v} t_{v}}{h_{1} t_{1}}-2 \frac{t_{v}}{h_{1}}\left(\frac{h_{v}}{h_{1}}\right)^{2} \frac{\cos \beta_{0}}{\operatorname{sen} a_{1}}\right] \varepsilon \\
& -\frac{6}{h_{1} t_{1}}\left(\frac{L}{h_{1} \pi}\right)^{2}\left[+M_{2}\left(\operatorname{ctg} a_{1}+\operatorname{ctg} a_{2}+\frac{n_{1}}{h_{2} \operatorname{sen} a_{2}}\right)-M_{3} \frac{n_{1}}{h_{2} \operatorname{sen} a_{2}}\right] \\
& =\frac{3}{t_{1}}\left(\frac{L}{n_{1} \pi}\right)^{2}\left[W_{0} \cos \beta_{0} \frac{n o}{n_{1}}\left(\operatorname{ctg} a_{1}+\operatorname{ctg} a_{2}\right)+\left(2 W_{0}+W_{1}+2 W_{2}\right) \frac{\cos \beta_{0}}{\operatorname{sen} a_{1}}-\left(W_{1}+W_{2}\right) \frac{\cos \beta_{2}}{\operatorname{sen} a_{2}}\right]
\end{aligned}
$$

La ecuación anterior es aplicable al punto 1 . Podemos obtener otra ecuación para el punto 0 . 
Sustituyendo la expresión de $\mathrm{P}_{0}$ que nos da la ecuación [26c], en la ecuación [24c], tenemos:

$$
2 t_{0}+f_{1}=-\frac{6}{t_{0}}\left(\frac{L}{\pi h_{0}}\right)^{2}\left(V_{1}-P_{v}\right) \frac{\cos \beta_{1}}{\operatorname{sen} \alpha_{1}}
$$

que por medio de las ecuaciones [26a] y [26e] se reduce a:

$$
\begin{aligned}
& 2 f_{0}+\left(1+\frac{t_{v}}{t_{0}}\left(\frac{h_{v}}{h_{0}}\right)^{2} \frac{\cos \beta_{1}}{\operatorname{sen} \alpha_{1}}\right) f_{1}+\frac{2 t_{v}}{t_{0}}\left(\frac{h_{v}}{h_{0}}\right)^{2} \frac{\cos \beta_{1}}{\operatorname{sen} \alpha_{1}} f_{v}+\frac{6}{t_{0} h_{0}}\left(\frac{L}{\pi h_{0}}\right)^{2} \frac{h_{0}}{h_{1} \operatorname{sen} \alpha_{1}} M_{2} \\
= & -\frac{3}{t_{0}}\left(\frac{L}{\pi h_{0}}\right)^{2}\left[\left(2 w_{0}+w_{1}+2 w_{v}\right) \frac{\cos \beta_{1}}{\operatorname{sen} \alpha_{1}}+w_{0} \frac{h_{0} \cos \beta_{0}}{h_{1} \operatorname{sen} \alpha_{1}}\right]
\end{aligned}
$$

Puesto que la presencia de la placa $\mathrm{V}$ introduce una nueva incógnita, la tensión longitudinal en $\mathrm{V}$, se precisa otra ecuación para el punto $\mathrm{V}$. Esta ecuación la podemos obtener basándonos en la compatibilidad de los desplazamientos en el punto 1. De la figura A9, obtenemos:

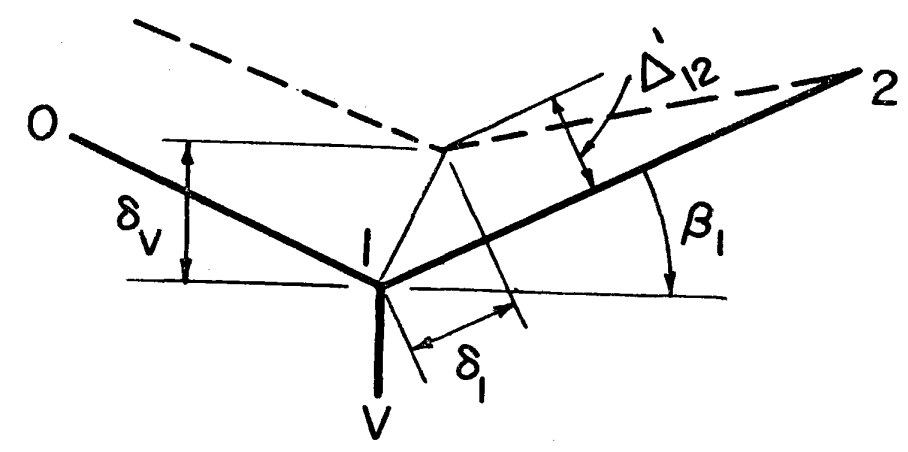

A9

$$
\delta_{v}=\delta_{1} \operatorname{sen} \beta_{1}-\Delta_{12}^{\prime} \cos \beta_{1}
$$

la cual, mediante la relación que aparece en la fórmula [5c], se reduce a:

$$
\delta_{v}=8, \operatorname{sen} \beta_{i}-\left(\delta_{0} / \operatorname{sen} \alpha_{1}-\delta_{1} \operatorname{ctg} \alpha_{1}\right) \cos \beta_{1}
$$

e introduciendo la identidad:

$$
\alpha_{1}=\beta_{1}-\beta_{0}
$$

se reduce $\mathbf{a}$ :

$$
\delta_{v}=\frac{\delta_{1} \cos \beta_{0}}{\operatorname{sen} \alpha_{1}}-\frac{8_{0} \cos \beta_{1}}{\operatorname{sen} \alpha_{1}}
$$


Expresando la flecha en función de las tensiones:

$$
\frac{f_{1}-f_{v}}{n_{v}}=\frac{f_{1}-f_{2}}{n_{1}} \frac{\cos \beta_{0}}{\operatorname{sen} \theta_{1}}-\frac{f_{0}-f_{1}}{n_{0}} \frac{\cos \beta_{1}}{\operatorname{sen} \alpha_{1}}
$$

Reduciendo términos semejantes:

$$
=\frac{n_{V}}{n_{0}} \frac{\cos \beta_{1}}{\operatorname{sen} a_{1}}-\left(1-\frac{n_{V}}{n_{0}} \frac{\cos \beta_{1}}{\operatorname{sen} a_{1}}-\frac{n_{v}}{n_{1}} \frac{\cos \beta_{0}}{\operatorname{sen} a_{1}}\right) f_{1}-\frac{n_{V}}{n_{1}} \frac{\cos \beta_{0}}{\operatorname{sen} a_{1}}+f_{1}=0
$$

Puesto que las ecuaciones en un pliegue cualquiera contienen términos relacionados con el estado tensional de otros pliegues vecinos, la presencia de la placa vertical en el pliegue 1 tiene su influencia sobre la relación entre momentos, tensiones y cargas en el pliegue 2. Una revisión de los procesos que nos han llevado a obtener la ecuación [8] mostrará que, en realidad, no se afecta esa ecuación. Sin embargo, la ecuación [16] debe modificarse, puesto que la sencilla relación establecida para la carga sobre la placa en la ecuación [15a] ya no es válida. En este punto, el valor de $\mathrm{P}$, dado por la ecuación [26e], es el que debe usarse. Debe hacerse notar que la única diferencia entre las ecuaciones [26c] y [15a], teniendo en cuenta el cambio de subíndices para transformar la fórmula para su aplicación al pliegue 2, es:

$$
\left[w_{v}+\frac{t_{v}}{6}\left(\frac{h_{v u}}{L}\right)^{2}\left(f_{1}+2 f_{v}\right)\right] \frac{\cos \beta_{0}}{\operatorname{sen} a_{1}}
$$

En consecuencia, multiplicando este término por $6 \mathrm{~L}^{2} / h_{1} \pi^{2}$ la acuación comparable a la [16], sin pretensado, $\in \mathbf{s :}$

$$
\begin{aligned}
& \left(\frac{h_{1} t_{1}}{h_{2} t_{2}}+\frac{t_{v} h_{v}^{2} \cos \beta_{0}}{t_{2} h_{1} h_{2} \operatorname{sen} a_{1}}\right) f_{1}+2\left(1+\frac{h_{1} t_{1}}{h_{2} t_{2}}\right) f_{2}+f_{3}+2 \frac{t_{v} h_{v}^{2} \cos \beta_{0}}{t_{2} h_{1} h_{2} \operatorname{sen} a_{1}} \\
& +\frac{6}{t_{2} h_{2}}\left(\frac{L}{\pi h_{2}}\right)^{2}\left(-C_{2}^{1} M_{1}+C_{2}^{2} M_{2}-C_{2}^{3} M_{3}+C_{2}^{4} M_{4}\right) \\
& =\frac{-3}{t_{2}}\left(\frac{L}{\pi h_{2}}\right)^{2}\left[\left(W_{0}+W_{1}\right) \frac{h_{2} \cos \beta_{0}}{h_{1} \operatorname{sen} a_{1}}-\left(W_{1}+W_{2}\right)\left(\frac{\cos \beta_{1}}{\operatorname{sen} a_{2}} \frac{h_{2} \cos \beta_{2}}{h_{1} \operatorname{sen} a_{2}}\right)+\left(W_{2}+W_{3}\right) \frac{\cos \beta_{3}}{\operatorname{sen} a_{3}}\right. \\
& \left.+2 \frac{\left.W_{v} h_{2} \cos \beta_{0}\right]}{n_{1} \operatorname{sen} a_{1}}\right]
\end{aligned}
$$

Debe hacerse notar que:

$$
\ldots 1=-\frac{W_{0} h_{0} \cos \beta_{0}}{2}
$$

Mediante todo este proceso previo de obtención de ecuaciones, es posible, con un método directo o indirecto de resolución de un sistema de ecuaciones, determinar las tensiones longitudinales y los momentos de todos los pliegues. Además de esto, es de desear una comprobación de la magnitud del esfuerzo transversal-tangencial en las placas $y_{\text {。 }}$ cuando sea excesivo, 
disponer la armadura adecuada para resistir la tensión diagonal. Ahora bien, la determinación de la tensión tangencial en un punto cualquiera puede obtenerse mejor por un procedimiento que consta de dos etapas. En este proceso debe tenerse en cuenta que la integral de las tensiones tangenciales en una sección cualquiera de una placa, debidas a los esfuerzos tangenciales longitudinales que actúan a la largo de los bordes, es nula. Esto puede confirmarse si tomamos un elemento aislado de una placa determinada, solicitada exclusivamente por tangenciales longitudinales en los dos bordes. El efecto de estos esfuerzos es producir tensiones en las fibras de estas placas.

Como estas tensiones varían a lo largo de la longitud de la placa, aparecen unos tangenciales paralelos al plano de la misma. Sin embargo, y puesto que no hay fuerzas normales al plano de la placa, por puras consideraciones estáticas, la integral de estos tangenciales que actúan en una sección cualquiera de una placa debe ser igual a la suma de los producidos por la fuerza normal $P$.

La ecuación [13] da la relación entre los esfuerzos que actúan en dos placas y las tensiones en las fibras de estas placas. En consecuencia, aunque se hubiesen determinado las tensiones, la magnitud de las fuerzas $\mathrm{P}$ no puede obtenerse directamente. Sin embargo, empezando en la primera placa exterior, utilizando la ecuación [13] puede reducirse hasta que contenga únicamente una $P$ desconocida. En resumen: $P_{0}$ se determina, en primer lugar, por la ecuación [17]. Entonces, con este valor $\mathrm{P}_{0}$ conocido, se puede calcular $\mathrm{P}$, a partir de la ecuación [13]. Los valores sucesivos de $\mathrm{P}$ pueden determinarse por orden. A partir de estos valores de $\mathrm{P}$, tenemos que el esfuerzo tangencial que actúa en cualquier sección de una placa determinada es:

$$
S_{n}^{T}=\frac{L}{\pi} P_{n} \cos \frac{\pi x}{L}
$$

Puesto que los elementos se suponen homogéneos, la distribución teórica de los tangenciales será parabólica, variando desde cero en un borde hasta un valor máximo en el eje neutro y pasando de nuevo a cero en el otro borde. Para las necesidades prácticas de proyecto es válido el método corriente de tratar el esfuerzo cortante, considerando como tensión media el valor:

\section{$S_{n}^{\top} 0.87+h$}

En unos pocos casos, excepcionales, en la región de los pliegues exteriores, puede presentarse una concentración de tangenciales, junto al doblez, debida al efecto de los esfuerzos tangenciales longitudinales en los bordes. La magnitud de estos tangenciales longitudinales puede determinarse partiendo, como para las fuerzas $P$, de la placa exterior, de acuerdo con la ecuación [24d]. Para el pliegue 2, sin viga vertical en el punto 1:

$$
f_{1}+f_{2}=\frac{2}{t_{2} h_{2}}\left(\frac{h}{\pi}\right)\left(s_{2}-s_{1}\right)+f_{12}^{p}+f_{21}^{p}
$$

en la cual ya es conocido el valor de $S_{1}$. La sus itución de los valores conocidos en esta ecuación da, naturalmente, la intensidad del tangencial en el pliegue. Con una viga vertical en el punto 1, para determinar $S_{21_{1}}$ es necesario, en primer lugar, calcular $S_{10}$ por medio de la ecuación [24f].

Conocidos $\mathrm{S}_{10}$ y $\mathrm{S}_{1 v}$, se obtiene $\mathrm{S}_{12}$ de la ecuación [24h]. Sustituyendo los valores conocidos de $f_{11}, f_{22}$ y $S_{12}$ en la ecuación [35], obtendremos el valor de $\mathrm{S}_{21}$. 
Con las fórmulas convencionales puede demostrarse que la variación del tangencial en una sección de una viga rectangular, debida a una distribución sinusoidal del esfuerzo tangencial a lo largo de un borde, es:

$$
S=S_{n}\left[1-4\left(\frac{y}{n}\right)+3\left(\frac{y}{n}\right)^{2}\right] \cos \frac{\pi x}{L}
$$

donde $y$ se mide a partir del borde cargado. En la figura A10 aparece una interpretación gráfica de la fórmula [36]. Debe hacerse notar que el tangencial disminuye desde un valor máximo en el borde cargado hasta ser nulo en el otro borde. El valor máximo del tangencial a la dirección opuesta se presenta para $y / h=0,67$.

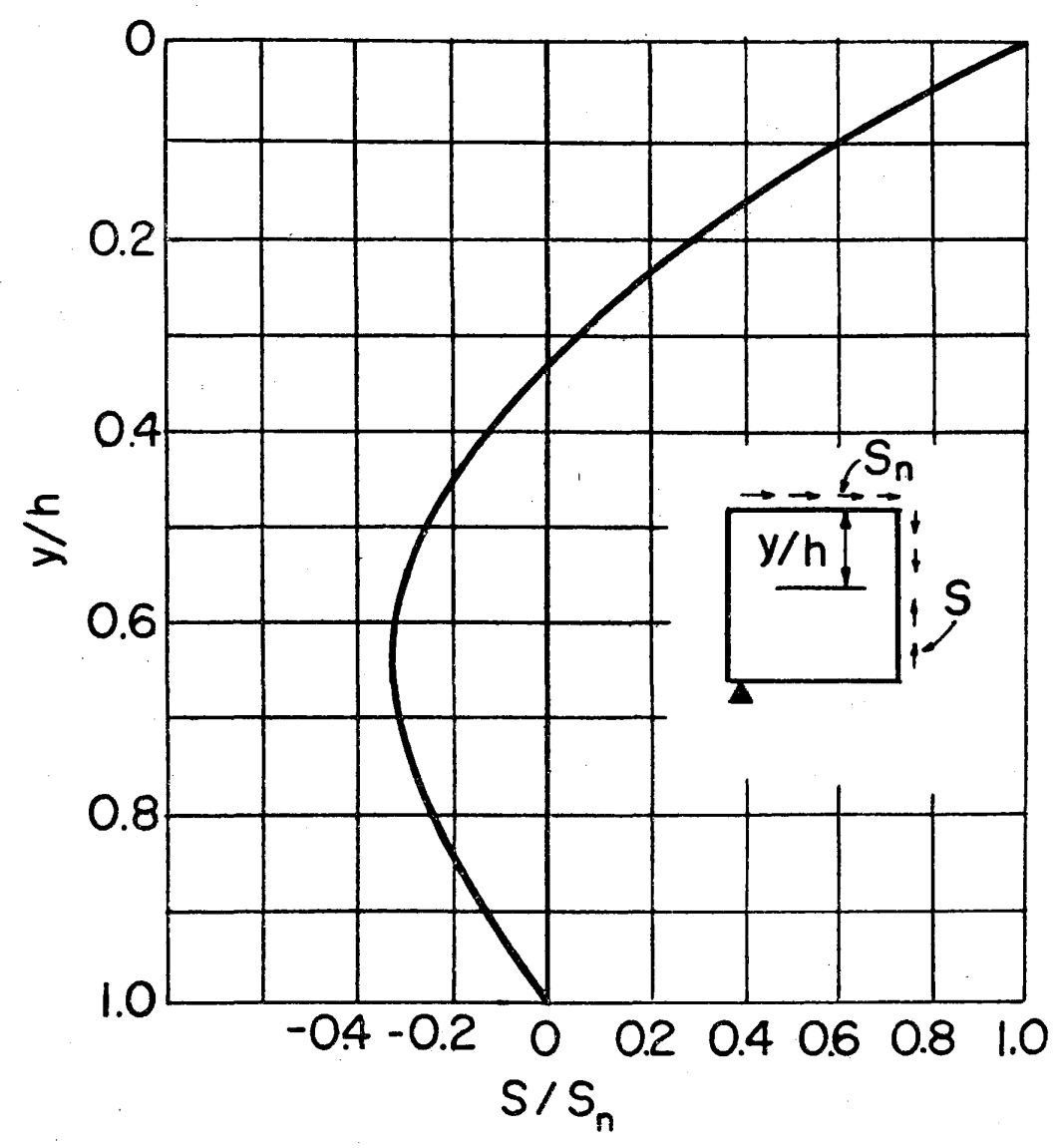

